\section{To: (Receiving Organization) Distribution}

5. Proj./Prog./Dept./Div.: Project $W-314 /$ TWRS

8. Originator Remarks:

This Design Review Report (QD) documents the contractor design verification methodology and records associated with Project $W-314^{\mp} \mathrm{s}$ "200 East Upgrades" design package.

11. Receiver Remarks: 11A. Design Baseline Document? [] Yes [X] No
3. From: (originating Organization) Tank Farm Restoration and Safe Operations Project

6. Design Authority/ Design Agent/Cog. Engr.:

(CAB 4/13/98

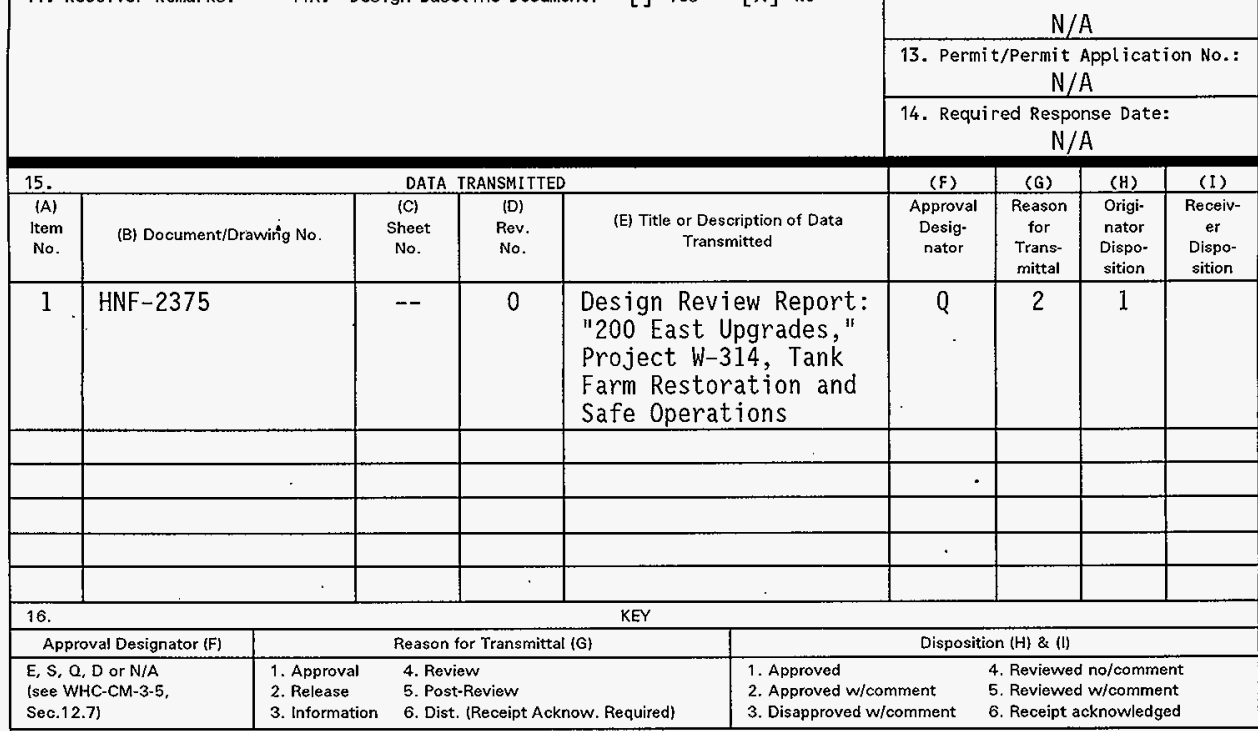

17. SIGNATURE/DISTRIBUTION

(See Approval Designator for required signatures)

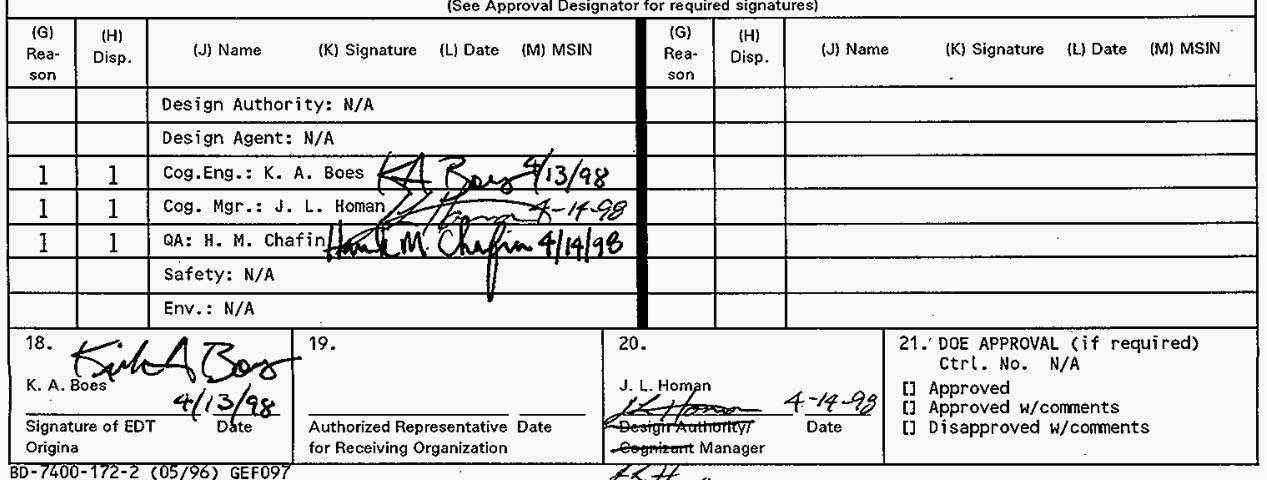

BD-7400-172-2 (05/96) GEF097

स2+7 4-14-98

4. Related EDT No.:

$\mathrm{N} / \mathrm{A}$

7. Purchase order No.:

$\mathrm{N} / \mathrm{A}$

9. Equip./Component No.:

$\mathrm{N} / \mathrm{A}$

10. System/Bldg./Facility: 200E Tank Farms

12. Major Assm. Dwg. No.: $N / A$ $N / A$ N/A 


\title{
Design Review Report: 200 East Upgrades for Project W-314, Tank Farm Restoration and Safe Operations
}

\author{
K. A. Boes \\ Numatec Hanford Corporation, Richland, WA 99352 \\ U.S. Department of Energy Contract DE-AC06-96RL13200 \\ EDT/ECN: $\quad 624066$ \\ Org Code: $8 C 430$ \\ UC: 510 \\ Charge Code: N311B \\ B\&R Code: EW 3130010 \\ Tota1 Pages: 72
}

Key Words: Design Review Report, DRR, W-314, Tank Farm Restoration and Safe Operations, Phase 1, 200 East Upgrades, Design Verification

Abstract: This Design Review Report (DRR) documents the contractor design verification methodology and records associated with project $W-314^{\prime} s$ "200 East (200E) Upgrades" design package. The DRR includes the documented comments and their respective dispositions for this design. Acceptance of the comment dispositions and closure of the review comments is indicated by the signatures of the participating reviewers.

TRADEMARK DISCLAIMER. Reference herein to any specific commercial product, process, or service by trade name, trademark, manufacturer, or otherwise, does not necessarily constitute or imply its endorsement, recommendation, or favoring by the United States Government or any agency thereof or its contractors or subcontractors.

Printed in the United States of America. To obtain copies of this document, contact: Document Control Services, P.0. Box 950, Mailstop H6-08, Richland WA 99352, Phone (509) 372-2420; Fax (509) 376-4989.
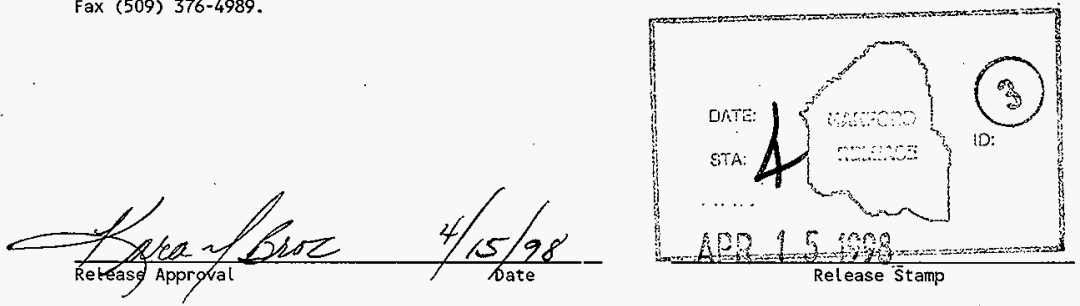
HNF-2375

Rev. 0

\title{
Design Review Report
}

\section{East Upgrades}

\author{
Project W-314 \\ Tank Farm Restoration and Safe Operations
}

Prepared by Numatec Hanford Corporation

Apri1 1998 
HNF-2375

Rev. 0

\section{CONTENTS}

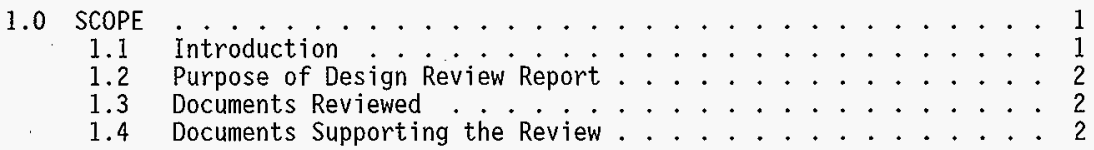

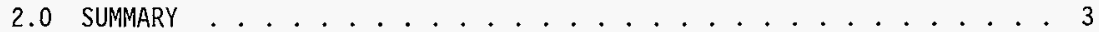

2.130 -Percent Design Review .................. 3

2.260 -Percent Design Review.................. . 4

2.3 Final Design Review ..................... 4

2.4 Approval of Design Media . . . . . . . . . . . . . . 5

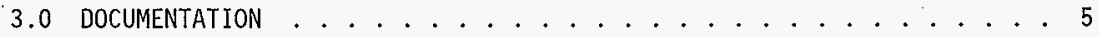

\section{ATTACHMENTS}

Attachment 1: List of 200 East Valve Pit Upgrades Design Media

Attachment 2: List of DRT Members

Attachment 3: Meeting Minutes Associated With 30-Percent Design Review

Attachment 4: Review Comment Records for 30-Percent Design Review

Attachment 5: Attendance Lists for 60-Percent Design Review

Attachment 6: Meeting Minutes Associated with Final Design Review

Attachment 7: Review Comment Records for Final Design Review

Attachment 8: EDT for Release of 200 East Valve Pit Upgrades Deșign Media 
HNF-2375, Rev. 0

Page 1 of 5

\section{DESIGN REVIEW REPORT}

\section{PROJECT W-314, TANK FARM RESTORATION AND SAFE OPERATIONS "200 EAST UPGRADES"}

\subsection{SCOPE}

\subsection{Introduction}

Project W-314, "Tank Farm Restoration and Safe Operations," is a Fiscal Year 1997 line item project within the Tank Waste Remediation System (TWRS) Tank Waste Retrieval Program. This project provides capital upgrades for the existing Hanford tank farms' waste transfer, instrumentation, ventilation, and electrical infrastructure systems. To support established TWRS programmatic objectives, the project is organized into two distinct phases. The initial focus of the project (i.e., Phase 1 ) is on waste transfer system upgrades needed to support the TWRS Privatization waste feed delivery system. Phase 2 of the project will provide upgrades to support resolution of regulatory compliance issues, improve tank infrastructure reliability, and reduce overal1 plant operating/maintenance costs.

Within Phase 1 of the W-314 project, the waste transfer system upgrades are further broken down into six major packages which align with the project's work breakdown structure. Each of these six sub-elements inciudes the design, procurement, and construction activities necessary to accomplish the specific tank farm upgrades contained within the package. The first design package ("AN Valve Pit Upgrades") was completed in November 1997, and the associated design verification activities are documented in HNF-1893. The second design package, "200 East (200E) Upgrades," was completed in March 1998. This design package identifies modifications to existing valve pits $241-A X-B$ and $241-A-B$, as well as several new waste transfer pipelines to be constructed within the A Farm Complex of the 200E Area. The scope of the valve pit modifications includes new pit cover blocks, valve manifolds, leak detectors, and special protective coatings similar to those previously approved for the AN Valve Pit Upgrades design package. The new transfer lines included in this package are to be routed (with official line number designations) as follows:

- From valve pit 241-AN-B to pump pit 241-AZ-02A (SN-630)

- From pump pit 241-AZ-02A to valve pit 241-AX-B (SN-632)

- From valve pit 241-AX-B to pump pit 241-AY-02A (SN-633)

- From Valve pit $241-\mathrm{AX}-\mathrm{B}$ to valve pit 241-A-B (SN-634)

In the case of 1 ines $\mathrm{SN}-630,-632$, and -633 , the piping tie-in interfaces with the existing pump pits are to be made outside the pits. The associated pit wall nozzles and piping stub-outs from the wall are to be performed under separate Phase I design/construction packages. 
HNF-2375, Rev. 0

Page 2 of 5

\subsection{Purpose of Design Review Report}

This Design Review Report (DRR) was prepared to document the Contractor's design verification methodology and records in accordance with HNF-PRO-445, Rev. 0, "Design Verification Requirements." The format and content for this report is based upon the applicable guidance contained in WHC-IP-1026, Appendix G.

\subsection{Documents Reviewed}

Design documents covered by this report include the definitive design drawings and construction specification associated with the W-314 200E Upgrades package. This design media addresses the total scope of the Project W-314 upgrades associated with the 241-AX-B and 241-A-B valve pits, as well as new transfer 1 ines $\mathrm{SN}-630, \mathrm{SN}-632, \mathrm{SN}-633$, and $\mathrm{SN}-634$. Additional $W-314$ Phase 1 modifications associated with the new transfer lines (i.e., piping tie-ins to valve pit 241-AN-B and to pump pits 241-AZ-02A and 241-AY-02A) are not included in the 200E Upgrades design package. Verification of the design for this scope will be performed and documented separately.

\subsection{Documents Supporting the Review}

The primary documents used to support the review of this design package were the Project Development Specifications (PDSs) listed below, along with associated Engineering Change Notices (ECNs). The PDSs, as modified by the ECNs, contain the total suite of approved design requirements for the W-314 Phase 1 activities.

- HNF-SD-W314-PDS-001, Rev. 0, "Project Development Specification for Transfer Piping"

- HNF-SD-W314-PDS-002, Rev. 0, "Project Development Specification for Valve Pit Manifold"

- HNF-SD-W314-PDS-003, Rev. 0, "Project Development Specification for Pit Leak Detection"

- HNF-SD-W314-PDS-005, Rev. 0, "Project Development Specification for Special Protective Coating"

Preparation of the definitive design media (drawings, specifications, etc.) for this package was performed between March 1997 and March 1998. During this period, customer reviews of the design deliverables were performed both formally and informally. These reviews included a formal review of the Piping and Instrumentation Diagrams (P\&IDs) at the 30-percent design completion stage, an informal review of the total "in-process" package at the 60-percent completion stage, and a formal review of all the design drawings and specifications at the design completion stage. Informal reviews of selected in-process design products were also performed on a bi-weekly basis. 
HNF-2375, Rev. 0

Page 3 of 5

\subsection{SUMMARY}

Formal reviews of the $W-314200 E$ Upgrades design package were conducted by a committee of personnel representing various disciplines and organizations. The Design Review Chairman was designated by the $W-314$ Project Manager. Other members of the Design Review Team (DRT) were selected based on their particular area of expertise and/or organizational affiliation. Personnel selected to participate as DRT members were not involved in performing the design work. A listing of the DRT personnel is provided in Attachment 1 .

\subsection{0-Percent Design Review}

A formal review of the new $241-A X-B$ and $241-A-B$ valve pit P\&IDs was conducted at the 30-percent design stage for the 200E upgrades package. Since the P\&IDs establish the configuration of the new waste transfer piping (manifolds) to be provided within the pits, finalization of this configuration, and customer buy-in to the P\&IDs, was the first key step in completing the overall design of this package.

The W-314 Design Review Team (DRT) convened on June 3, 1997 for the 30-percent design review briefing. During this briefing, the Design Agent (Fluor Daniel Northwest [FDNW]) distributed copies of the 30-percent design package to the DRT personne1. This package consisted of the P\&IDs to be formally reviewed by the Team, as well as "in-process" drawings (i.e., at various stages of completion) provided for informal review/comment. The FDNW Design Team presented their overal1 design approach, discussed significant features associated with the design, and answered questions posed by the DRT members.

On June 17, 1997, the DRT and the Design Team reconvened in the design review meeting to discuss comments associated with the 30-percent design package. As requested during the briefing two weeks earlier, the reviewers brought their review comments/questions to the meeting and presented them to the group as each of the design documents was reviewed. The FDNW Design Team and the DRT discussed and, to the greatest extent possible, dispositioned all the comments associated with the P\&IDs during this meeting. Comments which were dispositioned as "accepted," or which were determined to require further evaluation, were recorded by the secretary on a consolidated Review Comments Record (RCR) form representing the formal comments of the DRT. At the conclusion of the P\&ID review, the attendees informaliy reviewed each of the "in-process" design documents and discussed significant comments/questions concerning these products. These comments were not recorded on the RCR, but were instead noted by the FDNW Design Team for resolution prior to the final design review.

Minutes from the 30-percent design review briefing and design review meeting are included in Attachment 2. 
Following final resolution of a11 the comments on the formal RCR and concurrence with these dispositions by the DRT, approval signatures were obtained on the RCR from each of the key reviewers. Approval of the dispositioned RCR comments by the each of reviewing organizations constitutes closure of the review comments and DRT concurrence with the revised P\&IDs. A copy of the final RCR for the 30-percent review is provided in Attachment 3.

\subsection{0-Percent Design Review}

An informal review of the in-process design package was conducted at the 60 percent design stage. The 60-percent design package was distributed to the DRT on September 30, 1997, and the review team reconvened to discuss their comments with the FDNW Design Team on October 14, 1997. Since this review was conducted informally, no RCR documentation was prepared for the 60-percent review. Similar to the in-process design review conducted at the 30-percent stage, the attendees informally reviewed each of the in-process drawings for the 200E Upgrades package and discussed significant comments/questions concerning these products. These comments were noted by the FDNW Design Team for resolution prior to the final design review. Participants in the 60 -percent review briefing and the 60-percent review meeting are identified in Attachment 4 .

\subsection{Final Design Review}

A final design review was performed against FDNW's completed design products, which included all design drawings (e.g., P\&IDs, site plans, arrangement drawings, assemb7y drawings, etc.) and the construction specification. The same basic approach, as discussed above for the 30-percent review of the P\&IDs, was utilized to verify the completed design media and to document the formal comments.

The final design packages were distributed to the DRT at a briefing conducted on January 29, 1998. Following a two-week review period, the DRT and FDNW Design Team met again on February 12-13, 1998 to review and disposition the DRT's comments. Minutes from the review briefing and final design review meeting are included in Attachment 5.

Based on the approved comment dispositions reflected in the RCR for the final review (see Attachment 6), the $200 \mathrm{E}$ Upgrades design package was finalized by the Design Agent and approved for procurement/construction by the customer. 
HNF-2375, Rev. 0

Page 5 of 5

\subsection{Approval of Design Media}

There are no outstanding actions resulting from either the 30-percent or finat design reviews. Design verification of the $200 \mathrm{E}$ Upgrades design package is therefore considered to be complete. Engineering Data Transmittal (EDT) \#617948 officially issued/released the 200E Upgrades design media for construction. A copy of this EDT is included as Attachment 7.

Any design changes implemented for this package will be design verified prior to implementing the change.

\subsection{DOCUMENTATION}

The following supporting documentation is provided within the attachments to this DRR:

- Attachment 1 - List of DRT Members

- Attachment 2 - Meeting Minutes Associated With 30-Percent Design Review

- Attachment 3 - Review Comment Records for 30-Percent Design Review

- Attachment 4 - Attendance Lists for 60-Percent Design Review

- Attachment 5 - Meeting Minutes Associated With Final Design Review

- Attachment 6 - Review Comment Records for Final Design Review

- Attachment 7 - EDT \#617948 for Release of 200E Upgrades Design Media 
DESIGN REVIEW TEAM FOR W-314 "200E UPGRADES" (PHASE 1)

\begin{tabular}{|c|c|}
\hline ORGANIZATION/RESPONSIBILITY & REVIEWER NAME \\
\hline $\begin{array}{l}\text { W-314 Project Management } \\
: \quad \text { W-314 Technical Lead } \\
\text { - W-314 SE Management } \\
\text { W-314 Startup/Testing }\end{array}$ & $\begin{array}{l}\text { K. A. (Kirk) Boes (NHC) } \\
\text { D. L. (Dick) McGrew (NHC) } \\
\text { W. H. (Bi17) Hays (FDNW) } \\
\end{array}$ \\
\hline $\begin{array}{l}\text { DST Engineering } \\
\text { - W-314 Design Authority } \\
\text { - Transfer System Design Authority }\end{array}$ & $\begin{array}{l}\text { D. E. (Dave) Bowers (LMHC) } \\
\text { D. W. (Dan) Reberger (LMHC) }\end{array}$ \\
\hline $\begin{array}{l}\text { TWRS Equipment Engineering } \\
\text { : TWRS Design Authority } \\
\text { - Leak Detection Design Authority } \\
\text { - Electrical Design } \\
\text { Structural Design } \\
\end{array}$ & $\begin{array}{l}\text { S. H. (Shafik) Rifaey (LMHC) } \\
\text { C. C. (Chuck) Scaief (LMHC) } \\
\text { G. (Ghurdian) Singh (NHC) } \\
\text { M. A. (Mark) Scott (SESC) } \\
\end{array}$ \\
\hline Tank Waste Operations & R. W. (Jake) Jacobson (LMHC) \\
\hline Operations \& Projects Support & $\begin{array}{l}\text { T. K. (Treta) Ravencraft (LMHC) } \\
\text { G. A. (GTenn) Hester (LMHC) }\end{array}$ \\
\hline Rad Engineering/Tech Support & J. E. (Jim) Pieper (LMHC) \\
\hline SST Engineering & $\begin{array}{l}\text { D. R. (Dan) Nunamaker (LMHC) } \\
\text { M. (Mazen) A1-Wazani (LMHC) }\end{array}$ \\
\hline Maintenance Program Integration & J. R. (Jim) LaPointe (LMHC) \\
\hline Quality Assurance & H. M. (Hank) Chafin (FDNW) \\
\hline Operations Compliance & P. C. (Phil) Miller (LMHC) \\
\hline Nuclear Safety & S. U. (Shakir) Zaman (LMHC) \\
\hline Operations \& Project Safety Support & M. S. (Matt) Tiffany (DESH) \\
\hline TWRS Testing \& Syștems Readiness & R. L. (Ron) Legg (LMHC) \\
\hline Process Development & J. D. (John) Galbraith (NHC) \\
\hline
\end{tabular}


Subject: $30 \%$ DESIGN REVIEW BRIEFING - W-314 200E UPGRADES

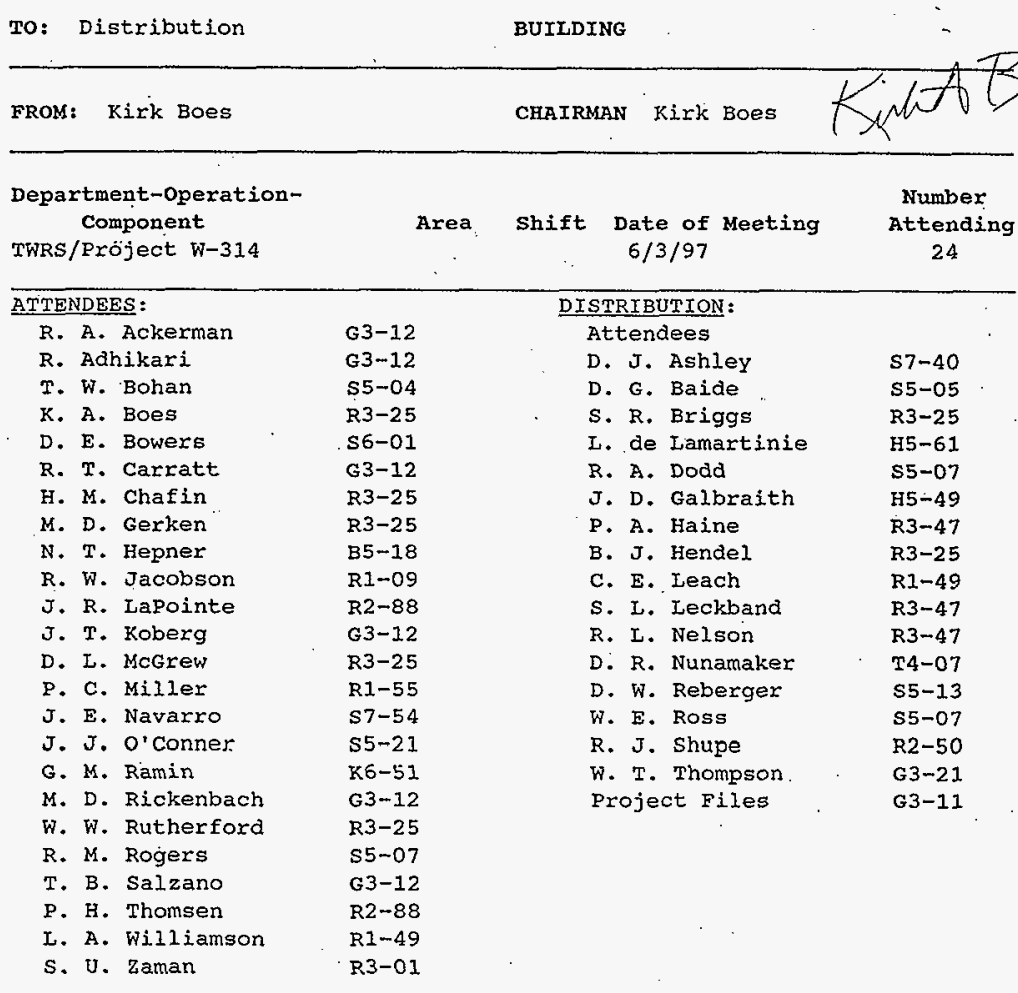

John Koberg welcomed the assembled Design Review Team (attendees identified in Attachment 1) and opened the meeting by presenting the $30 \%$ complete design package for the $\mathrm{W}-314200 \mathrm{E}$ Upgrades.

The scope of the 200E Upgrades package includes modifications to the 241-A-A, 241-A-B, 241-AX-A, and 241-AX-B valve pits. These modifications include new valve pit manifold assemblies and cover blocks, replacement pit leak detectors, special protective coating for the pits, and replacement of selected encasement line leak detectors. Also included in the scope of this design package are three separate waste transfer pipelines needed to support waste transfer operations in the A Farm Complex.

Kirk Boes noted that East Tank Farm Operations had recently identified several recommendations to the project concerning the manifold configurations and schedule. These recommendations are documented in IMHC letter \#77100-97-044. Information copies of this letter were distributed to the meeting attendees. Kirk stated that change control documentation is currently being prepared to address the changes 
identified in the Operations letter. These pending changes are not reflected in the 30 \% design review package.

John stated that the $30 \%$ review will be conducted in similar fashion to the one that was recently completed using the same Design Review Team for the W-314 AN Valve Pit upgrades (April 1997). The Piping and Instrumentation Diagrams (P\&IDs) included in the design package are considered to be complete and are being submitted for formal design review by the Design Review Team. All other design products contained in the package are considered to be "in-process" documents (i.e., currently at various stages of completion) and are being presented to the Team for informal review/comment. The Design Review Team will reconvene in two weeks to present and discuss their comments on the $30 \%$ design package. The FDNW Design Leads and the Design Review Team will resolve/disposition all comments against the P\&IDs during this meeting. Comments which are dispositioned as "accepted," or which require further evaluation by the Design Team, will be recorded by the secretary on a consolidated Review Comments Record (RCR) form representing the formal comments of the team. In general, comments which are dispositioned as "not accepted" will not be recorded on the RCR. Closure of this RCR will signify the Design Review Team's approval of the P\&IDS. At the conclusion of the PAID review, the attendees will review each of the inprocess documents and discuss any significant comments/questions concerning these products.

The products depicted in the $200 \mathrm{E}$ Upgrades $30 \%$ review package are very similat to the those reviewed previously by the Design Review Team for the $\mathrm{W}-314$ AN Valve Pit Upgrades package. The contents of the $200 \mathrm{E}$ Upgrades $30 \%$ review package are listed in Attachment 2 . Specific items within the package were presented to the assembled Design Review Team by the FDNW Design Leads, as follows:

- Tom Salzano (Piping Lead Engineer) discussed the completed P\&IDs associated with the new $A-A, A-B$, and $A X-B$ valve pit manifolds (drawings H-14-101079, -101080, -101047, and -101048), and briefly described the other in-process jumper and piping drawings.

- Richard Ackerman (Civil/structural Lead Engineer) reviewed the inprocess drawings associated with the new valve pit cover blocks, valve pit modifications, and pit demolition work.

- Dick Carratt (Instrumentation Lead Engineer) briefly reviewed the in-process drawings associtated with the valve pit instrumentation.

- Ron Adhikari (Electrical Lead Engineer) reviewed the in-process electrical drawings associated with the cathodic protection system modifications.

The in-process Construction specification associated with the $200 \mathrm{E}$ Upgrades was not included in the $30 \%$ design review package since it is very similar to the one presented to the review team at the previous $30 \%$ review conducted for the AN Valve Pit Upgrades design package. Gil Ramin asked whether other design-related products, such as calculations, would be issued for review. John Koberg and Kirk Boes explained that design calculations are not typically issued as part of a design review package. Calculations and other supporting documentation may be provided to specific reviewers qualified to review the results, or to others upon request. 
As with the previous $30 \%$ review conducted for $w-314$ in April, this review for the $200 E$ Upgrades P\&IDs and in-process design documents shall be based on the requirements established in the approved project Development specifications (PDSs). The applicable pDSs for this design package are as follows: .

- HNF-SD-W314-PDS-001, Rev. 0: Transfer Piping

- HNF-SD-W314-PDS-002, Rev, 0: Valve Pit Manifolds

- HNF-SD-W314-PDS-003, Rev. 0: Pit Ieak Detection

- HNF-SD-W314-PDS-005, Rev. 0: special Protective coating

John Koberg ciosed the $30 \%$ design review briefing by encouraging the Design Review Team members to feel free to contact any of the FDNW Design Leads (i.e., Rickenbach, Ackerman, Adhikari, Carratt, Salzano) if any further explanation or clarification of the design products/philosophy is needed to support their review.

ACTION ITEM: The $W-314$ Design Review Team will reconvene at $7: 30$ am in the TCPC Building/4th floox conference room on Tuesday, $6 / 17 / 97$, to review/disposition the Team's comments on the 200E Upgrades design package.

ATPACHMENT 1: 30\% Design Review Briefing Attendance List ATTACHMENT 2: $30 \%$ Design Package Contents 
ATTACHMENT

HNF-2375, Rev. 0

ATTACHMENT 2

$6 / 2 / 47$

$30 \%$ Design Renew $200 \mathrm{k} / \mathrm{w}$ Package

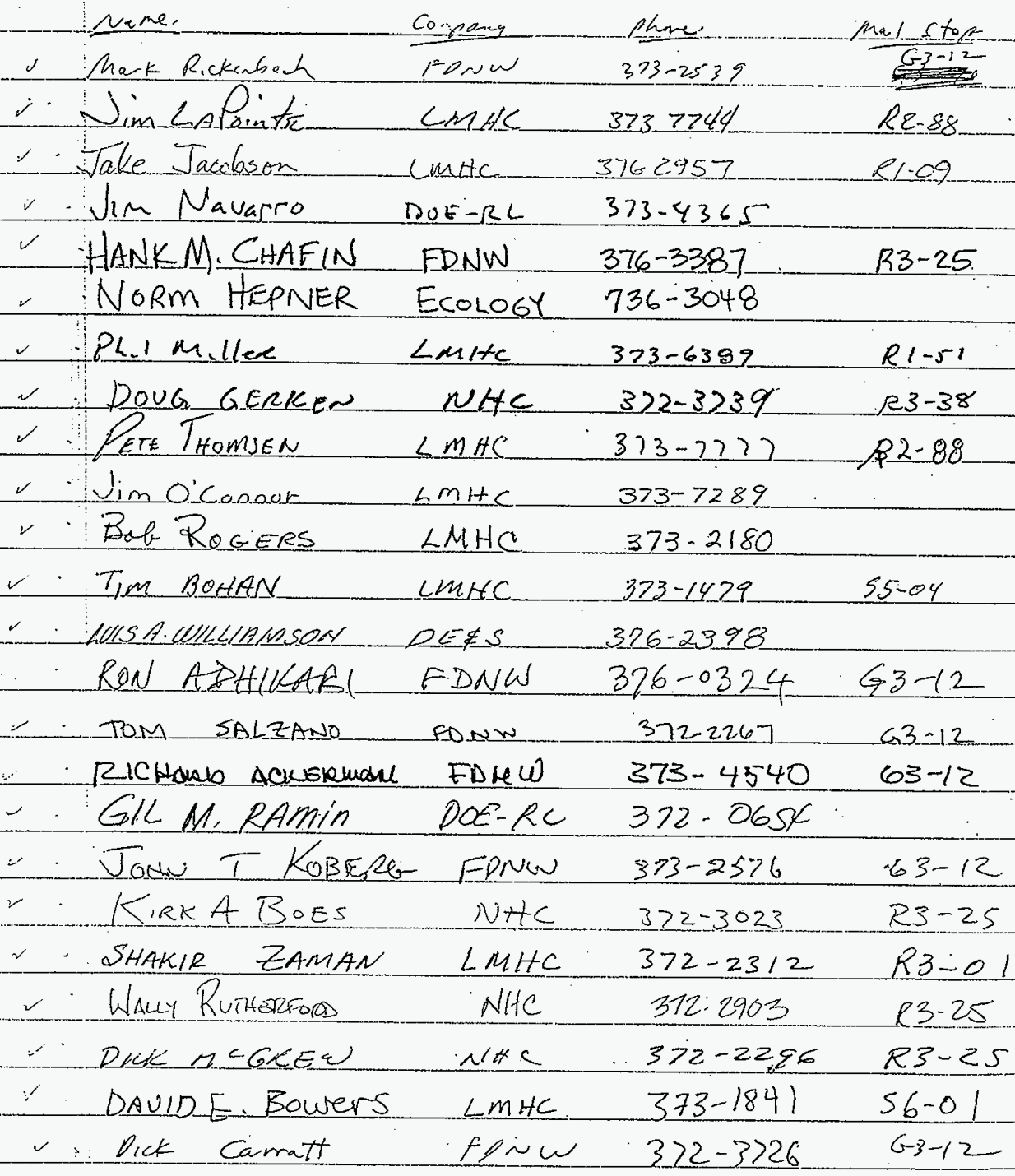




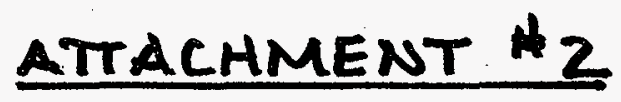

HNF-2375, ReV. 0 ATTACHMENT 2

A2-5

W-314 200E Upgrades - 308 Design Review Package

\begin{tabular}{|c|c|c|}
\hline Drawing No. & Sheet & Title \\
\hline$H-14-101047$ & 1 & P\&ID Valve Pit $241-A X-B$ \\
\hline $\mathrm{H}-14-101048$ & 1 & P\&ID Misc. Details \\
\hline $\mathrm{H}-14-101079$ & 1 & P\&ID Valve Pit $241-A-A$ \\
\hline $\mathrm{H}-14-101080$ & 1 & P\&ID Valve Pit $241-A-B$ \\
\hline $\mathrm{H}-14-101081$ & 1 & P\&ID Misc. Details \\
\hline$H-14-100999$ & 1 & Structural Cover Blocks 241-AX-B \\
\hline $\mathrm{H}-14-101000$ & 1 & Structural Cover Blocks.Sections and Details \\
\hline $\mathrm{H}-14-101002$ & 1 & Structural Cover Blocks 241-A-B Plan \\
\hline $\mathrm{H}-14-101003$ & 1 & Structural 241-AC-B \& 241-A-B Wall Modifications \\
\hline$H-14-101020$ & $1-3$ & Cathodic Protection Plan Valve Pit 241-AN-B \\
\hline $\mathrm{H}-14-101023$ & 1 & Cathodic Protection Details \\
\hline $\mathrm{H}-14-101023$ & 1 & Cathodic Protection Details \\
\hline $\mathrm{H}-14-101028$ & 1. & Inst. $241-A \& 241-A x$ Plan \\
\hline $\mathrm{H}-14-101029$ & 1 & Inst. AX Farm Valve Pit Plan 24I-AX-A \& 241-AX-B \\
\hline $\mathrm{H}-14-101030$ & 1 & Inst. 241-AX-B Elevation \& Details \\
\hline$H-14-101031$ & 1 & Inst. A Farm Valve Pit Plan 241-A-A \& 241-A-B \\
\hline $\mathrm{H}-14-101032$ & 1 & Inst. 241-A-B Elevation \& Details \\
\hline$H-14-101051$ & 1 & Jumper Arrangement Valve Pit $241-A X-B$ \\
\hline $\mathrm{H}-14-101052$ & 1 & Jumper Arrangement Valve Pit 241-AX-B Section \\
\hline$H-14-101061$ & $1-2$ & Jumper AssembIy 241-AX-B R16-R19-R20-(D\&E) \\
\hline $\mathrm{H}-14-101062$ & 1 & Jumper Assembly 241-AX-B \\
\hline $\mathrm{H}-14-101063$ & 1 & Jumper Assembly 241-AX-B RI-(E) \\
\hline$H-14-101064$ & 1 & Jumpex Assembly 241-AX-B R11-R12-(A) \\
\hline $\mathrm{H}-14-101065$ & $1-2$ & Jumper Assembly VP-10OA R3 \\
\hline$H-14-101066$ & 1 & Jumper Assembly 241-AX-B R1A-Drain \\
\hline $\mathrm{H}-14-101067$ & 1 & Jumper Assembly 241-AX-B R3A-Drain \\
\hline $\mathrm{H}-14-101071$ & $1-4$ & Funnel Assembly 2-Way and 3-Way Valves - Details \\
\hline $\mathrm{H}-14-101074$ & $1-4$ & Piping Valve Pit 241-AX-B Hodification Details \\
\hline $\mathrm{H}-14-101076$ & 1 & Pit Demolition Jumper Pit $241-\mathrm{AX}-\mathrm{B}$ \\
\hline $\mathrm{H}-14-101077$ & 1 & Valve Actuator Details \\
\hline $\mathrm{H}-14-101082$ & 1 & Jumper Arrangement Valve Pit $241-\mathrm{A}-\mathrm{A}$ \\
\hline $\mathrm{H}-14-010184$ & 1 & Jumper Arrangement Valve Pit $241-A-B$ \\
\hline$H-14-010185$ & 1 & Jumper Arrangement Valve Pit $241-A-B$ section \\
\hline $\mathrm{H}-14-101094$ & $1-2$ & Jumper Assembly 241-A-B R1-R2-(A) \\
\hline $\mathrm{H}-14-101095$ & $1-2$ & Jumper Assembly 241-A-B R15-R16-R19-(B) \\
\hline$H-14-101096$ & $1-2$ & Jumper Assembly $241-A-B$ (A) $-(B)-(C)$ \\
\hline $\mathrm{H}-14-101097$ & $1-2$ & - Jumper Assembly 241-A-B R3-R9-(D) \\
\hline $\mathrm{H}-14-101103$ & 1 & Pit Cover Painting Diagram 241-A-B \\
\hline$H-14-101110$ & $1-3$ & Piping Plan Valve Pit 241-AN-B \\
\hline
\end{tabular}


Subject: $30 \%$ DESIGN REVIEW MEETING - W-314. 200E UPGRADES

To: Distribution

FROM; Kirk Boes

\section{BUIIDING}

\begin{tabular}{|c|c|c|c|}
\hline $\begin{array}{l}\text { Department-operation- } \\
\text { Component } \\
\text { TWRS/Project } \mathrm{W}-314\end{array}$ & Area & $\begin{array}{c}\text { Shift } \begin{array}{c}\text { Date of Meeting } \\
6 / 17 / 97\end{array}\end{array}$ & $\begin{array}{l}\text { Number } \\
\text { Attending } \\
20\end{array}$ \\
\hline $\begin{array}{l}\text { ATTENDEES: } \\
\text { R. A. Ackerman } \\
\text { R. Adhikari } \\
\text { E. L. Ahola } \\
\text { K. A. Boes } \\
\text { D. E. Bowers } \\
\text { R. T. Carratt } \\
\text { H. M. Chafin } \\
\text { M. D. Gerken } \\
\text { J. R. LaPointe } \\
\text { J. T. Koberg } \\
\text { D. L. McGrew } \\
\text { D. R. Nunamaker } \\
\text { R. S. McMurphy } \\
\text { J. E. Navarro } \\
\text { M. D. Rickenbach } \\
\text { R. M. Rogers } \\
\text { W. W. Rutherford } \\
\text { T. B. Salzano } \\
\text { L. A. Williamson } \\
\text { J. A. Wright }\end{array}$ & $\begin{array}{l}\text { G3-12 } \\
\text { G3-12 } \\
\text { E6-27 } \\
\text { R3-25 } \\
\text { S6-01 } \\
\text { G3-12 } \\
\text { R3-25 } \\
\text { R3-25 } \\
\text { R2-88 } \\
\text { G3-12 } \\
\text { R3-25 } \\
\text { T4-07 } \\
\text { S5-50 } \\
\text { S7-54 } \\
\text { G3-12 } \\
\text { S5-07 } \\
\text { R3-25 } \\
\text { G3-12 } \\
\text { R1-49 } \\
\text { S5-50 }\end{array}$ & $\begin{array}{l}\text { DISTRIBUTION: } \\
\text { Attendees } \\
\text { D. J. Ashley } \\
\text { D. G. Baide } \\
\text { S. R. Briggs } \\
\text { T. W. Bohan } \\
\text { L. de Lamartinie } \\
\text { R. A. Dodd } \\
\text { J. D. Galbraith } \\
\text { P. A. Haine } \\
\text { B. J. Hendel } \\
\text { N. T. Hepner } \\
\text { C. E. Leach } \\
\text { S. L. Leckband } \\
\text { R. W. Jacobson } \\
\text { P. C. Miller } \\
\text { R. I. Nelson } \\
\text { J. J. O'Conner } \\
\text { D. W. Reberger } \\
\text { W. E. Ross } \\
\text { R. J. Shupe } \\
\text { P. H. Thomsen } \\
\text { W. T. Thompson } \\
\text { S. U. Zaman } \\
\text { Project Files }\end{array}$ & $\begin{array}{l}\text { S7-40 } \\
\text { S5-05 } \\
\text { R3-25 } \\
\text { S5-04 } \\
\text { H5-61 } \\
\text { S5-07 } \\
\text { H5-49 } \\
\text { R3-47 } \\
\text { R3-25 } \\
\text { B5-18 } \\
\text { R1-49 } \\
\text { R3-25 } \\
\text { R1-09 } \\
\text { R1-51 } \\
\text { R3-47 } \\
\text { S5-21 } \\
\text { S5-13 } \\
\text { S5-07 } \\
\text { R2-50 } \\
\text { R2-88 } \\
\text { G3-21 } \\
\text { R3-01 } \\
\text { G3-11 }\end{array}$ \\
\hline
\end{tabular}

These minutes document the proceedings from the $30 \%$ Design Review Meeting conducted on $6 / 17 / 97$ for Project $W-314$ 's 200E Upgrades definitive design. The $30 \%$ design package prepared by FDNW was distributed to the Design Review Team during the 6/3/97 Design Review Briefing. The purpose of this meeting was to formally review the completed Piping and Instrumentation Diagrams (P\&IDs) associated with the upgraded $241-A-B$ and $241-A X-B$ valve pits, and to informally review the other "in-process" design drawings associated with this work. As discussed during the meeting, the P\&IDs associated with the 241-A-A and 241-AX-A valve pit upgrades were not included in the formal review because these upgrades have been identified for deferral into Phase 2 of the project. Participants in the review meeting are identified in Attachment 1 . 
The following drawings were formally reviewed by the Design Review Team:

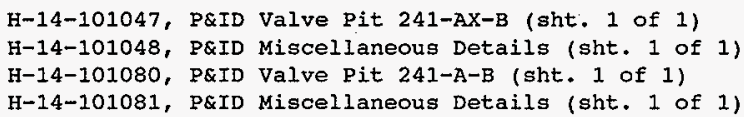

Comments were identified by the Design Review Team and discussed by the assembled participants. Comments dispositioned during the meeting as "accepted," or which were determined to require further evaluation by the Design Team, were recorded on a consolidated Review Comments Record (RCR) form. This RCR represents the formal comments of the team (see Attachment 2). Following resolution and/or incorporation of all the formal comments associated with the P\&IDs, each of the reviewing organizations will sign-off the RCR to signify closure of the Design Review Team's comments and acceptance of the P\&IDs. It is intended that these completed PQIDs will set the basic design configuration for the $A X-B$ and $A-B$ valve pit upgrades, and provide the basis for completing the remaining design details.

Review comments associated with the in-process documents (see list of reviewed items in Attachment 3) were not recorded on an RCR. Instead, these comments were identified by the design reviewers and then discussed by the assembled group. In most cases, the reviewers. comments were either accepted for incorporation or were determined to be a result of the current incomplete/"unchecked" condition of the design media. The informal comments were recorded as "red-line" markups to the drawings and specification by the FDNW Design Team.

An additional interim review of the in-process design products is planned to be performed at the $60 \%$ complete stage for this design package. The 608 design review is scheduled to take place during early september 1997. The completed design documentation for the 200E Upgrades will be submitted for formal review by the Design Review Team upon completion by the FDNW Design Team. This final review of the $200 \mathrm{E}$ Upgrades design is currently planned to begin in early February 1998. Updated information concerning the $60 \%$ and final reviews for this design package will be issued to the $\mathrm{w}-314$ Design Review Team in advance of the review dates. As required to support the design verification documentation requirements (WHC-CM-6-1, EP-4.1), a Design Review Report containing all formal design review documentation associated with the $200 \mathrm{E}$ Upgrades will be issued at the conclusion of this definitive design activity.

Reviewers are reminded that additional informal in-process reviews of the w-314 design media are conducted during the bi-weekly Design status Meetings. For more information concerning these meetings, or any other information discussed in these minutes, please contact Kirk Boes at 372-3023.

ATTACHMENT 1: 308 Design Review Meeting Attendance List ATTACHMENT 2: P\&ID Review Comments ATTACHMENT 3: In-Process Design Documentation List 


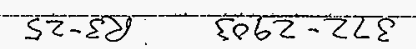

इZ-हय: $\quad$ कदzर-ZLह

टा-59:- $9 ट \Omega-5<5$

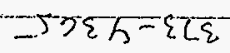

रा-हण: कडस-दूह

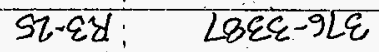
उह-95 कहरई- दरह

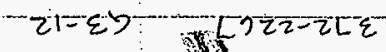

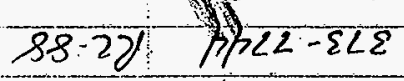

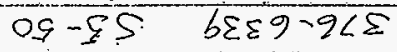
OS-SS $\quad 668 \varepsilon-9 \angle \varepsilon$ Zा-ह9 $92 L \varepsilon-2<\varepsilon$ OSTE-ELE

$77-\varepsilon 5$ tic $0-92 \Sigma$ $\angle Z-97$. हh $\angle Q-9 \angle \varepsilon$ ¿b80-948

to/2L. $\Omega / 16-\Sigma L \varepsilon$ $10-95$ ThPT-ZLE

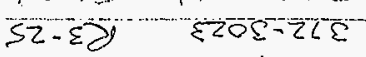

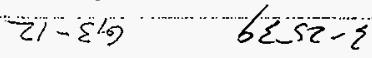

THN

JTN

लnd

751

लमबन

$M N O 1$.

$\rightarrow A T$

MNOS

Tामून

MNQI

nTNE=

mNOJ

गHWज

MNCS

MNOF

$5 \% 3 \mathrm{C}$

मापर

याग/गाw 7

कार-M/

mथन- axy=m2y $4110 \mathrm{M}$

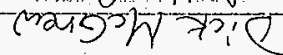

गयनड त्र तण लusron wi?

Towordra cropata

NIUH $=1 N \forall f$ catrso snod

ondzTys



मेंत्nायाओ roy परातल NसाO?

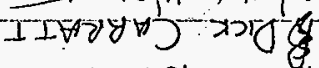

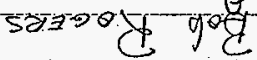

INATHE NOY

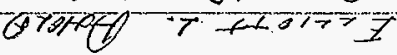

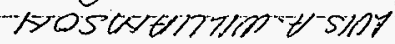
sapurion N N कालm jict 520870.9 Tropsiy rem
.2
-51

की

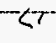

\section{? \\ ST

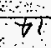

5

212

.ll

i

$-6$

$8-4$ 


\section{REVIEW COMMENT RECORD (RCR)}

1. Oate
$6 / 17 / 97$

3. Project No.

$\mathrm{W}-314$
2. Revien No. 200E/W-001

4. Page

\section{1 of 4}

\begin{tabular}{l|l|l}
\hline 5. Document Number(s)/Title(s) & $\begin{array}{l}\text { 6. Program/Project/ } \\
\text { Building Number } \\
\text { TwRS/Project } \\
\text { W-314 }\end{array}$ & 7. Reviewer \\
Project W-314, Phase 1-200E/W & See below \\
Upgrades P\&IDs: & & \\
H-14-101047, H-14-101048, H-14- & \\
101080, and H-14-101081 & \\
\hline
\end{tabular}

17. Comment Submittal Approval:

10. Agreement with indicated comment disposition(s)

8. Organization/Group
see below

9. Location/Phone
Date

Date

Date

Date

Date

Date

Date

Date
Facitity Design (W-314 Systems Engineering Lead)

Date 


\section{REVIEW COMMENT RECORD (RCR)}

\begin{tabular}{l} 
1. Date $6 / 17 / 97$ \\
\hline $3.9 r o j e c t ~$
\end{tabular}

3. Project No.

$\bar{W}-314$
2. Review No. 200E/W-001

4. Page

2 of 4

\begin{tabular}{|c|c|c|c|c|}
\hline $\begin{array}{l}12 . \\
\text { I tem }\end{array}$ & $\begin{array}{l}\text { 13. Comment(s)/Discrepancy(s) (Provide technical justification for the } \\
\text { comment and detailed recomendation of the action required to correct/ } \\
\text { resolve the discrepancy/problem indicated.) }\end{array}$ & $\begin{array}{l}14 . \\
\text { Hoid } \\
\text { Point }\end{array}$ & 15. Disposition (Provide justification if NOr accepted.) & $\begin{array}{l}16 . \\
\text { Status }\end{array}$ \\
\hline 1 . & $\begin{array}{l}\text { H-14-101047: Delete 2-inch manifold in the 241-AX-B } \\
\text { valve pit as per approved ECN } \# 162730 \text {. (Bowers) }\end{array}$ & & & \\
\hline 2. & $\begin{array}{l}\text { H-14-101047: Evaluate the need for both valves } \\
\text { (\#WT-V-001 and -015) shown in the manifold line } \\
\text { connected to nozzle R1. ( ) }\end{array}$ & & & \\
\hline 3. & $\begin{array}{l}\text { H-14-101047: Add appropriate safety classification } \\
\text { designation and additional notes to the PQID, as } \\
\text { required. This comment applies to all other } W-314 \\
\text { P\&IDs. (Chafin) }\end{array}$ & & & \\
\hline 4. & $\begin{array}{l}\text { H-14-101047: Delete 3-inch manifold connection to } \\
\text { nozzle R19 as per approved ECN \#162730 (manifold } \\
\text { connection to "cross-over line" between AX valve } \\
\text { pits is not needed). (Bowers) }\end{array}$ & & & \\
\hline 5. & $\begin{array}{l}\text { H-14-101047: Evaluate methods to minimize/eliminate } \\
\text { contamination in capped-off piping stub-outs to } \\
\text { facilitate ALARA during future construction tie-ins. } \\
\text { This comment also applies to similar activities } \\
\text { performed in other pits. (Wright) }\end{array}$ & & & \\
\hline 6. & $\begin{array}{l}\text { H-14-101047: Replace generic equipment numbers with } \\
\text { assigned TWRs equipment designations as applicable. } \\
\text { This comment also applies to H-14-101080. } \\
\text { (Rickenbach) }\end{array}$ & & & \\
\hline 7. & $\begin{array}{l}\text { H-14-101047: Consider moving symbol Legend and item } \\
\text { identification legend to the P\&ID misc. details } \\
\text { drawing. Comment also applies to drawing H-14- } \\
\text { 101080. (Bowers) }\end{array}$ & & . & \\
\hline 8. & $\begin{array}{l}\text { H-14-101047: Evaluate methods to eliminate } \\
\text { permanent manifold connection to line SL-502 which } \\
\text { is scheduled for isolation/replacement during phase } \\
2 \text { of the project. (Bowers) }\end{array}$ & & & \\
\hline
\end{tabular}




\section{REVIEW COMMENT RECORD (RCR)}

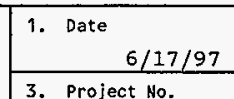

2. Review No.

3. Project No. 200E/W-001

$w-314$

\begin{tabular}{|c|c|c|c|c|}
\hline $\begin{array}{l}12 . \\
\text { Item }\end{array}$ & $\begin{array}{l}\text { 13. Comment(s)/Discrepancy(s) (Provide technical justification for the } \\
\text { conment and detailed recommendation of the action required to correct/ } \\
\text { resolve the discrepancy/problem indicated.) }\end{array}$ & $\begin{array}{l}\text { 14. } \\
\text { Hold } \\
\text { Point }\end{array}$ & 15. Disposition (Provide justification if NOr accepted.) & $\begin{array}{l}16 . \\
\text { Status }\end{array}$ \\
\hline 9. & $\begin{array}{l}\text { H-14-101047: Revise note associated with 241-AX-B } \\
\text { floor drain to identify the drain line destination } \\
\text { as Tank AY-102. (Bowers) }\end{array}$ & & $\because$ & \\
\hline 10. & $\begin{array}{l}\text { H-14-101047: Revise General Note \#2 as needed to } \\
\text { address SL-502 isolation and any other piping } \\
\text { changes reflected on this drawing. (Bowers) }\end{array}$ & $\cdot$ & . & \\
\hline 11 & $\begin{array}{l}\text { H-14-101047: Confirm that existing line } \mathrm{SN}-400 \text { is } \\
\text { actually isolated at } \mathrm{AX}-501 \text { pit as indicated. } \\
\text { (Bowers) }\end{array}$ & & & \\
\hline 12 & $\begin{array}{l}\text { H-14-101047: Delete "HOLD \#2" and insert } \\
\text { appropriate equipment number for hydrotest riser } \\
\text { associated with line SN-632. (Salzano) }\end{array}$ & & & \\
\hline 13 & $\begin{array}{l}\text { H-14-101047: Revise modifications shown for } \\
\text { instrument air purge line associated with line SL- } \\
100 \text { to eliminate cutting and capping of this line -- } \\
\text { no changes to be made to existing purge air line. } \\
\text { (Salzano) }\end{array}$ & & . & \\
\hline 14 & $\begin{array}{l}\text { H-14-101047: Ensure the nomenclature for the drain } \\
\text { line connected to nozzle R1A is consistent with the } \\
\text { same line shown on H-14-101051. (Miller) }\end{array}$ & & & \\
\hline 15. & $\begin{array}{l}\text { H-14-101047: Ensure label associated with the line } \\
\text { connected to nozzle R3 is consistent with the same } \\
\text { line shown on } H-14-101051 \text {. (Miller) }\end{array}$ & & & \\
\hline 16. & $\begin{array}{l}\text { H-14-101048: No comments except as noted above for } \\
\text { generic P\&ID changes. }\end{array}$ & & . & \\
\hline 17. & $\begin{array}{l}\text { H-14-101080: Evaluate methods to eliminate } \\
\text { "permanent" manifold connection to line SN-216 which } \\
\text { goes to the 244-A DCRT. (Bowers) }\end{array}$ & & & \\
\hline 18. & $\begin{array}{l}\text { H-14-101080: Delete 2-inch manifold in the } 241-A-B \\
\text { valve pit as per approved ECN } \# 162730 \text {. (Bowers) }\end{array}$ & & & \\
\hline
\end{tabular}




\section{REVIEW COMMENT RECORD (RCR)}

\begin{tabular}{l} 
1. Date $6 / 17 / 97$ \\
\hline
\end{tabular}

3. Project No.

พ-314
2. Review No. 200E/W-OO1

4. Page

4 of 4

\begin{tabular}{|c|c|c|c|c|}
\hline $\begin{array}{l}12 . \\
\text { Item }\end{array}$ & $\begin{array}{l}\text { 13. Comment(s)/0iscrepancy(s) (Provide technical justification for the } \\
\text { coment and detailed recommendation of the action required to correct/ } \\
\text { resolve the discrepancy/problem indicated.) }\end{array}$ & $\begin{array}{l}14 . \\
\text { Hoid } \\
\text { Point }\end{array}$ & 15. Disposition (Provide justification if NOT accepted.) & $\begin{array}{l}16 . \\
\text { Status }\end{array}$ \\
\hline 19. & $\begin{array}{l}\text { H-14-101080: Revise note associated with } 241-\mathrm{A}-\mathrm{B} \\
\text { floor drain to identify the drain line destination } \\
\text { as Catch Tank } \mathrm{A}-350 \text {. ( ) }\end{array}$ & & & \\
\hline 20 & $\begin{array}{l}\text { E-14-101080: Isolate air purge supply for sN- } \\
200 / 213 \text { line (line taken out of service and isolated } \\
\text { by this design) at its source, and remove associated } \\
\text { pressure switch tie-in to the master pump shutdown } \\
\text { system. () }\end{array}$ & & $\cdot$ & \\
\hline 21. & $\begin{array}{l}\text { H-14-101080: Revise General Note \#2 to address } \\
\text { piping changes reflected on this drawing. (Bowers) }\end{array}$ & & & \\
\hline 22 . & $\begin{array}{l}\text { H-14-101080: Revise note associated with sL-113 } \\
\text { line to indicate its destination as } 242-\mathrm{A} \\
\text { Evaporator. ( ) }\end{array}$ & & & \\
\hline 23. & $\begin{array}{l}\text { H-14-101080: Revise modifications shown for } \\
\text { instrument air purge line associated with line sN- } \\
219 \text { to eliminate cutting and capping of this line -- } \\
\text { no changes to be made to existing purge air line. } \\
\text { (Salzano) }\end{array}$ & & . & \\
\hline 24. & $\begin{array}{l}\text { H-14-101080: Correct typo in last sentence of } \\
\text { General Note \#1 (should be "identification"). } \\
\text { (Boes) }\end{array}$ & & & \\
\hline 25 & $\begin{array}{l}\text { H-14-101080: Ensure label associated with the line } \\
\text { connected to nozzle R1 is consistent with the same } \\
\text { line shown on H-14-101084. (Milier) }\end{array}$ & & & \\
\hline 26. & $\begin{array}{l}\text { H-14-101080: Ensure label associated with the line } \\
\text { connected to nozzle R14 is consistent with the same } \\
\text { line shown on H-14-101084. (Miller) }\end{array}$ & & & \\
\hline 27. & $\begin{array}{l}\text { H-14-101081: No comments except as noted above for } \\
\text { generic P\&ID changes. }\end{array}$ & & & \\
\hline
\end{tabular}




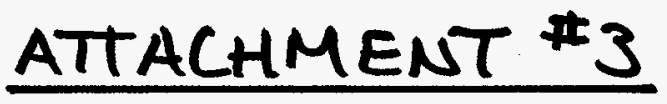

HNF-2375, Rev. 0

ATTACHMENT 2

A2-13

W-314 200E Upgrades - $30 \%$ Design Review Package

\begin{tabular}{|c|c|c|}
\hline Drawing No. & Sheet & Title \\
\hline $\mathrm{H}-14-101047$ & 1 & P\&ID Valve Pit $241-\mathrm{AX}-\mathrm{B}$ \\
\hline $\mathrm{H}-14-101048$ & 1 & P\&ID Misc. Details \\
\hline $\mathrm{H}-14-101079$ & 1 & P\&ID valve Pit $24,1-\mathrm{A}-\mathrm{A}$ \\
\hline $\mathrm{H}-14-101080$ & 1 & P\&ID Valve Pit $241-A-B$ \\
\hline $\mathrm{H}-14-101081$ & 1 & P\&ID Misc. Details \\
\hline$H-14-100999$ & 1 & Structural Cover Blocks 241-AX-B \\
\hline$H-14-101000$ & 1 & Structural Cover Blocks Sections and Details \\
\hline $\mathrm{H}-14-101002$ & 1 & Structural Cover Blocks 241-A-B Plan. \\
\hline $\mathrm{H}-14-101003$ & 1 & Structural 241-AC-B \& 241-A-B Wall Modifications \\
\hline $\mathrm{H}-14-101020$ & $1-3$ & Cathodic Protection Plan Valve Pit 241-AN-B \\
\hline $\mathrm{H}-14-101023$ & 1 & Cathodic Protection Details \\
\hline $\mathrm{H}-14-101023$ & 1 & Cathodic Protection Details \\
\hline $\mathrm{H}-14-101028$ & 1 & Inst. 241-A \& 241-AX Plan \\
\hline $\mathrm{H}-14-101029$ & 1 & Inst. $A X$ Farm Valve Pit Plan 241-AX-A \& 241-AX-B \\
\hline $\mathrm{H}-14-101030$ & 1 & Inst. 241-AX-B Elevation \& Details \\
\hline $\mathrm{H}-14-101031$ & 1 & Inst. A Farm valve Pit Plan 241-A-A \& 241-A-B \\
\hline $\mathrm{H}-14-101032$ & 1 & Inst. 241-A-B Elevation \& Details \\
\hline $\mathrm{H}-14-101051$ & 1 & Jumper Arrangement Valve Pit $241-A X-B$ \\
\hline $\mathrm{H}-14-101052$ & 1 & Jumper Arrangement Valve Pit $241-A X-B$ section \\
\hline $\mathrm{H}-14-101061$ & $1-2$ & Jumper Assembly 241-AX-B R16-R19-R20-(D\&E) \\
\hline $\mathrm{H}-14-101062$ & 1 & Jumper Assembly 241-AX-B \\
\hline $\mathrm{H}-14-101063$ & 1 & Jumper Assembly 241-AX-B Rl-(E) \\
\hline$H-14-101064$ & 1 & Jumper Assembly 241-AX-B R11-R12-(A) \\
\hline $\mathrm{H}-14-101065$ & $1-2$ & Jumper Assembly VP-10OA R3 \\
\hline$H-14-101066$ & 1 & Jumper Assembly 241-AX-B R1A-Drain \\
\hline $\mathrm{H}-14-101067$ & 1 & Jumper Assembly 241-AX-B R3A-Drain \\
\hline $\mathrm{H}-14-101071$ & $1-4$ & Funnel Assembly 2-Way and 3-Way Valves - Details \\
\hline$H-14-101074$ & $1-4$ & Piping valve Pit 241-AX-B Modification Details \\
\hline$H-14-101076$ & 1 & Pit Demolition Jumper Pit $241-\mathrm{AX}-\mathrm{B}$ \\
\hline$H-14-101077$ & 1 & Valve Actuator Details \\
\hline$H-14-101082$ & 1 & Jumper Arrangement Valve Pit $241-A-A$ \\
\hline $\mathrm{H}-14-010184$ & 1 & Jumper Arrangement Valve Pit 241-A-B \\
\hline$H-14-010185$ & 1 & Jumper Arrangement Valve Pit $241-A-B$ Section \\
\hline$H-14-101094$ & $1-2$ & Jumper Assembly 241-A-B R1-R2-(A) \\
\hline $\mathrm{H}-14-101095$ & $1-2$ & Jumper Assembly 241-A-B R15-R16-R19-(B) \\
\hline $\mathrm{H}-14-101096$ & $1-2$ & Jumper Assembly $241-A-B(A)-(B)-(C)$ \\
\hline$H-14-101097$ & $1-2$ & Jumper Assembly 241-A-B R3-R9-(D) \\
\hline $\mathrm{H}-14-101103$ & 1 & Pit Cover Painting Diagram 241-A-B \\
\hline $\mathrm{H}-14-101110$ & $1-3$ & Piping Plan Valve Pit 241-AN-B \\
\hline
\end{tabular}




\begin{tabular}{|c|c|}
\hline 1. Date & $\begin{array}{l}\text { 2. Review No. } \\
\text { 200E/W-001 }\end{array}$ \\
\hline $\begin{array}{c}\text { 3. Project No. } \\
\text { W-314 }\end{array}$ & $\begin{array}{l}\text { 4. Page } \\
1 \text { of } 1\end{array}$ \\
\hline
\end{tabular}

5. Document Number(s)/Title(s)

Project $W-314$, Phase 1 - 200E/W Upgrades P\&IDs:

$\mathrm{H}-14-101047, \mathrm{H}-14-101048, \mathrm{H}-14-$ 101080 , and $\mathrm{H}-14-101081$

17. Comment Submittal approval:

Facility Design (W-318 Engifeering Lead) $9 / 8 / 97$

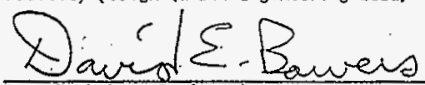

$9 / 9 / 97$ East Tank Farm Engineering

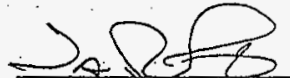

Maintenance Progran Integration

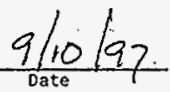

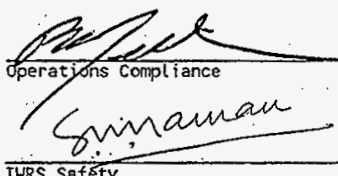

$9 / 9 / 87$

$9 / 10 / 97$

Date

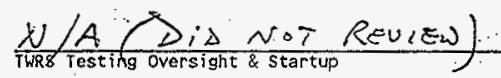

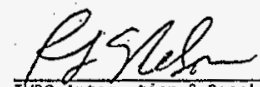

ThRs integration \& gasel ine Management

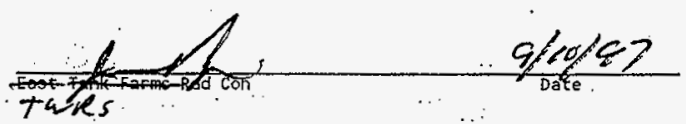

TWRS/Project

W- 314

7. Reviewer
See below

8. Organization/Group

See below

9. Location/Phone

See below

11. CLOSED
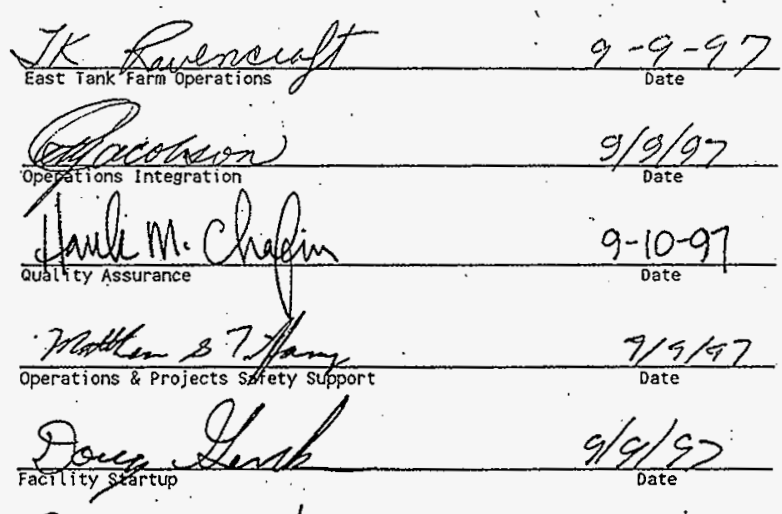

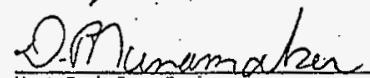

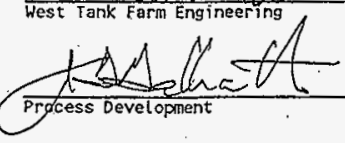

$9 / 9 / 97 \ldots$

(1)

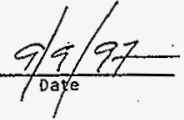

\footnotetext{
A-6600.090.1 (03/92)
} 


\begin{tabular}{|c|c|c|c|c|}
\hline \multirow{2}{*}{\multicolumn{3}{|c|}{ REVIEW COMMENT RECORD (RCR) }} & \multirow{2}{*}{\begin{tabular}{|c|} 
1. Date \\
$6 / 17 / 97$ \\
$\begin{array}{c}\text { 3. Project No. } \\
\text { W-314 }\end{array}$ \\
\end{tabular}} & $\begin{array}{l}\text { 2. Revien Ho. } \\
200 E / \mathrm{W}-00 \mathrm{I}\end{array}$ \\
\hline & & & & \\
\hline $\begin{array}{ll}12 . \\
\text { Item }\end{array}$ & $\begin{array}{l}\text { 13. Corment(s)/pis screpancy(s) (provide technical justification for the } \\
\text { comment and detai led recomendation of the action required to correct/ } \\
\text { resolve the discrepgncyforoblem indicated.) }\end{array}$ & $\begin{array}{l}\text { 1. } \\
\text { Rolo } \\
\text { Point } \\
\end{array}$ & 15. Disposition (Provide justification if NOT accepted.) & $\begin{array}{l}16 . \\
\text { status } \\
\end{array}$ \\
\hline 1. & $\begin{array}{l}\text { H-14-101047: Delete 2-inch manifold in the } 241-A X-B \\
\text { valve pit as per approved ECN \#162730. (Bowers) }\end{array}$ & & Accepted - deleted manifold. & \\
\hline 2. & $\begin{array}{l}\text { H-14-101047: Evaluate the need for both vaives } \\
\text { (\#WT-V-001 and -015) shown in the manifold Tine } \\
\text { connected to nozzle RI. }\end{array}$ & & Accepted - only one valve is required. & \\
\hline 3. & $\begin{array}{l}\text { H-14-101047: Add appropriate safety classification } \\
\text { designation and additional notes to the P\&I0, as } \\
\text { required. This comment applies to a11 other } W-314 \\
\text { P\&IDs. (Chafin) }\end{array}$ & & $\begin{array}{l}\text { Accepted-will identify drawing as "Safety } \\
\text { Class" with a clarifying note as to which } \\
\text { components are SC. }\end{array}$ & . \\
\hline 4. & $\begin{array}{l}\text { H-14-101047: Delete 3-inch manifold connection to } \\
\text { nozzle R19 as per approved ECN "t162730 (manifold } \\
\text { connection to "cross-over line" between AX valve } \\
\text { pits is not needed). (Bowers) }\end{array}$ & & Accepted - deleted manifold. & \\
\hline 5. & $\begin{array}{l}\text { H-14-101047: Evaluate methods to minimize/el iminate } \\
\text { contamination in capped-off piping stub-outs to } \\
\text { facilitate ALARA during future construction tie-ins. } \\
\text { This comment also app Ties to similar activities } \\
\text { performed in other pits. (Wright) }\end{array}$ & & $\begin{array}{l}\text { This is not an issue for the P\&ID to } \\
\text { address; however, the ALARA concern is noted } \\
\text { and this issue will be addressed during } \\
\text { detailed design of the pit modifications. }\end{array}$ & \\
\hline 6. & $\begin{array}{l}\text { H-14-101047: Replace generic equipment numbers with } \\
\text { assigned TWRS equipment designations as applicable. } \\
\text { This comment also app Ties to H-14-101080. } \\
\text { (Rickenbach) }\end{array}$ & & Accepted. & \\
\hline 7 . & $\begin{array}{l}\text { H-14-101047: Consider moving. Symbol Legend and item } \\
\text { identification legend to the P\&ID misc. details } \\
\text { drawing. Comment also appTies to drawing H-I4- } \\
101080 \text {. (Bowers) }\end{array}$ & & $\begin{array}{l}\text { Accepted - wi11 move symbol legend as } \\
\text { suggested. }\end{array}$ & \\
\hline 8. & $\begin{array}{l}\text { H-14-101047: Evaluate methods to el iminate } \\
\text { permanent manifold connection. to Iine } S L-502 \text { which } \\
\text { is scheduled for isolation/replacement during Phase } \\
2 \text { of the project. (Bowers) }\end{array}$ & & $\begin{array}{l}\text { Accepted - a temporary jumper is recommended } \\
\text { for this purpose and wil1 be provided, if } \\
\text { approved by a change request. }\end{array}$ & \\
\hline
\end{tabular}




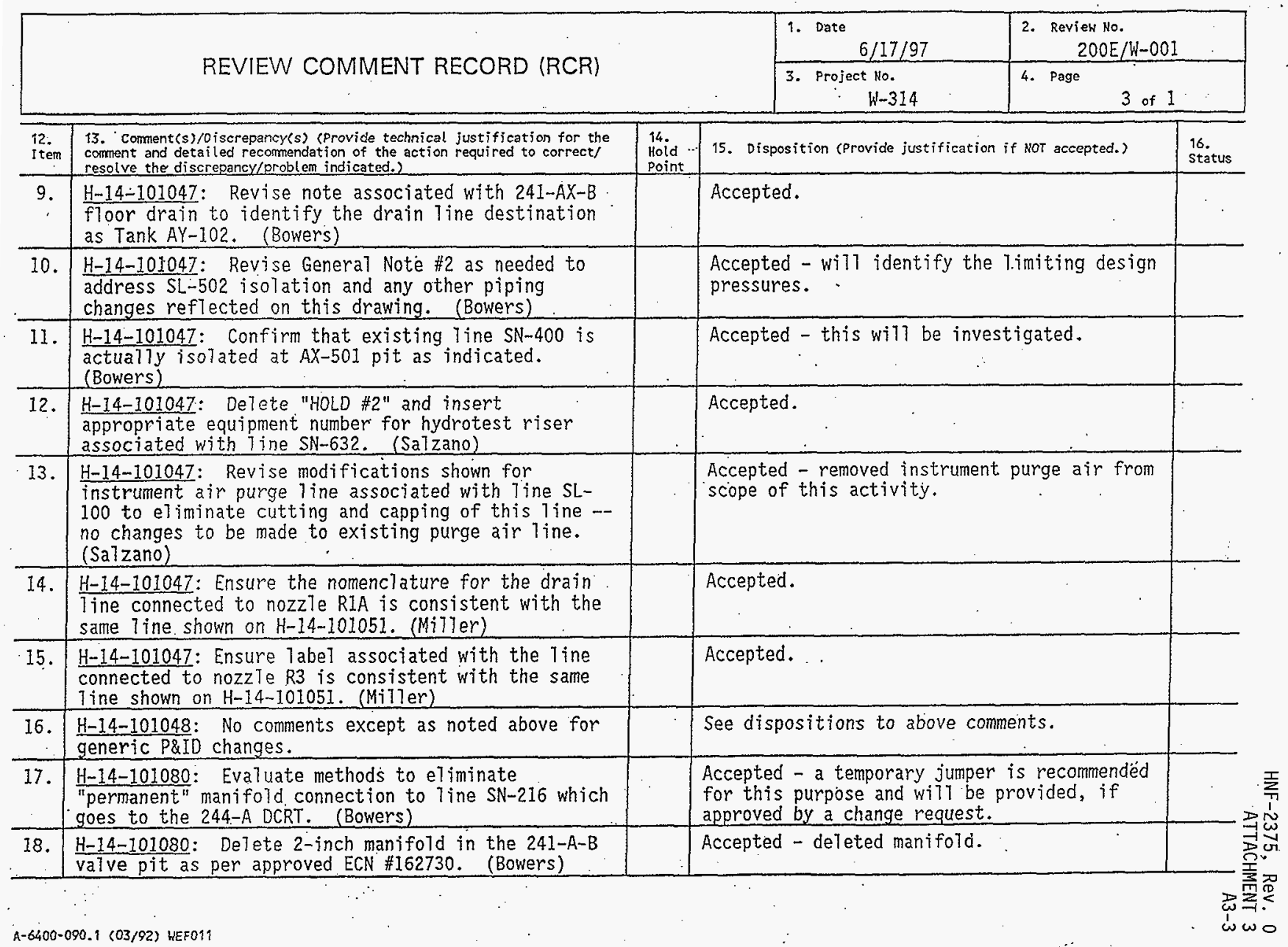




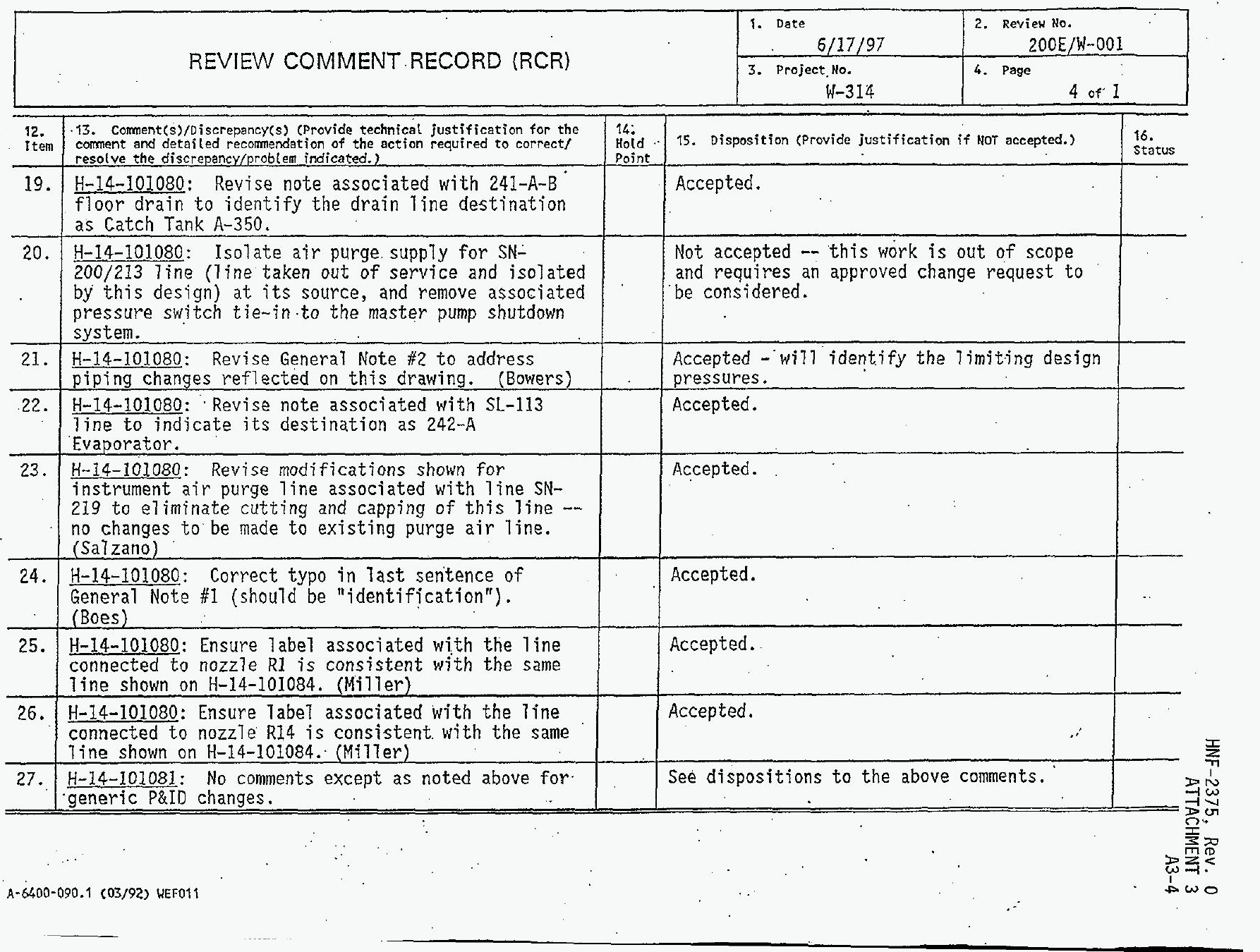




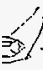

$9 / 30197$

HNF-2375, Rev. 0

ATTACHMENT 4

A4-1

$200 \mathrm{k} / \mathrm{w}$ Design Packeje. Go\% Revinw kackff

Mark Rackenach

Bou thays

Kirk Boes

DICK MCGRE
P.DW

Fonu

NHL/Projects

MHC/Projects.

LMHE. W WTE

LMItCL LORERTIONS 373 -9275

$3.73-2539$

376.0047

$3: 2.3023$

$372-2296$

Dan Nunamaker

Treta Ravencraft zmitclow ops comp

Phel Mellee

EDNW/QA

$376-3387$

HANK Chafin

FDNW

$372-2267$

TOM SALZANO

EDHeW

$373-4540$

RLCHOaE ACKERMBS

FDNCW

$376-0324$

FDNW $\quad 372-3726$

Dick Cannat

EONW

$373-2576$

JOC KOBERG

LMHC

$373-184)$

DAVID Bowers

FON w

373.3329

Shakir Zaman LMHC

Matt Tiffany DESH 
HNF-2375, Rev. 0

ATTACHMENT 4

$10 / 1+\langle q\rangle$

A4-2

$60 \%$ Resign Review Heeting - $200 \mathrm{E} / \mathrm{m}$ Racks

Mark Rickerbach ipuw

$373-2539$

DAj:

$372 \ldots 124$

deEF Whatam

EDNW

$376-6401$

RON ADHILARI

FDNW

$376-0324$

Treta havencraft

LMHC

$373-1275$

tames Pieper

LMAC

$3>64175$

Dan Niwamaker LMHC $\quad 373.9115$

DICK CARRATT EONW $372-3726$

MARKE.DALAS FDNW 376.2949

RICHAWE ACKERMall FDNW $373-4540$

TOM SALZANO

FDNW

$372-2267$

Jim Navarra

RL

$373-4365$

Matthes 5 Tiffany

PESH

$373 \cdot 2148$

Sin lapointo

LMHC

3737244

Ph. hidlen

LMHE

$323-6389$

TOHN KUBERO

FDIVW

$373-2576$

Kirk Boes

NHC

$372-3023$

Hank M. Chafin

FDNW

$376-3387$ 
Subject: FINAL DESIGN REVIEW BRIEFING - W-314 200E UPGRADES

T0: Distribution BUILDING

FRON: Kirk Boes

CHAIRMAN Kirk Boes

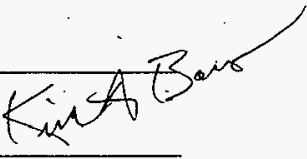

Department-operationComponent

TWRS/Project W-314

Area Shift Date of Meeting $1 / 29 / 98$
Number

Attending

18

\begin{tabular}{ll}
\hline ATTENDEES: & \\
\hline R. A. Ackerman & $G 3-12$ \\
R. Adhikari & G3-12 \\
K. A. Boes & R3-25 \\
D. E. Bowers & $S 5-13$ \\
M. E. Dallas & $\mathrm{G} 3-12$ \\
H. M. Chafin & R3-25 \\
W. J. Eischens & $S 7-73$ \\
W. H. Hays & E6-08 \\
R. W. Jacobson & R1-09 \\
E. H. Leschber & $S 7-73$ \\
P. C. Miller & RI-51 \\
D. L. McGrew & R3-25 \\
J. E. Navarro & $S 7-54$ \\
J. E. Pieper & $S 5-21$ \\
M. D. Rickenbach & G3-12 \\
S. H. Rifaey & RI-56 \\
T. B. Salzano & G3-12 \\
C. C. Scaief & R1-56
\end{tabular}

DISTRIBUTION:

Attendees

D. G. Baide

T. W. Bohan

R. T. Carratt.

R. A. Dodd

J. D. Galbraith

P. A. Haine

J. L. Homan

J. T. Koberg

J. R. LaPointe

S. L. Leckband

R. L. Nelson

D. R. Nunamaker

D. W. Reberger

W. W. Rutherford

T. K. Ravencraft

W. T. Thompson

M. S. Tiffany

S. U. Zaman

Project Files
S5-05

S5-04

G3-12

S5-07

H5-49

R3-47

T4-07

G3-12

R2-88

R3-47

R3-47

T4-07

S5-13

R3-25

S5-04

G3-21

R1-49

S5-12

G3-11

These minutes document the discussions and actions from the final definitive design review kickoff briefing for Project W-314's "200E Upgrades" design package. This package is the second of six planned definitive design packages for Phase 1 of the $W-314$ project to be completed.

Following general introductions, Mark Rickenbach (FDNW Principle Lead Engineer) opened the meeting by presenting an overview of the $200 \mathrm{E}$ Upgrades. The scope of the $200 \mathrm{E}$ Upgrades package consists of modifications to the 200E waste transfer. system in support of TWRS Privatization and tank farm operations, including: upgrades to valve pits 241-AX-B and 241-A-B (i.e., new valve pit manifold assemblies and cover blocks; new pit nozzles; new pit leak detectors; special protective coating for the pits; new encasement line leak detectors; and associated instrumentation/electrical work), and new waste transfer pipelines between the following locations:

- Valve pit 241-AN-B to pump pit 241-AZ-02A

- Pump pit 241-AZ-02A to Valve pit 241-AX-B

- Valve pit $241-A X-B$ to valve pit $241-A-B$

- Valve pit 241-AX-B to pump. pit 241-AY-02A 
Copies of the completed definitive design package, consisting of

drawings (119 sheets) and the W-314-C2 Construction Specification, were distributed to the attendees. Specific deliverables contained within the design package were presented to the assembled Design Review Team by the FDNW design personnel, as follows:

\section{Civil/Structural Drawings - Richard Ackerman}

- Richard Ackerman presented the piping plans and profile drawings, structural coverblock and wall modification drawings for the $A X-B$ and $A-B$ pits, and other miscellaneous civil/structural drawings included in the package (H-14-100994 through H-14-101002).

- Dave Bowers (W-314 Design Authority) indicated that an initial comment concerning the piping $\mathrm{pl}$ ans and profile drawings is that these drawings should include identification of line designations for all existing lines crossed by the new W-314 Tines. Ackerman said that this information is currently shown on the piping layout drawings and can easily be added to the plans and.profiles.

\section{Electrical Drawings - Ron Adhikari}

- Ron Adhikari presented the electrical site plans and installation detail drawings, electrical panelboard schedule, wire run 1 ist, and cathodic protection drawings (H-14-101008 through $\mathrm{H}-14-101024)$.

\section{Instrumentation Drawings - Mark Dallas}

- Mark Dallas described instrumentation and contral drawings associated with the $A X-B$ and $A-B$ valve pits, including the instrumentation plan and elevation drawings, loop diagrams (for valve pit leak detectors, eccasement leak detectors, and vaive position sensors), and instrument lists (H-14-101028 through $\mathrm{H}-14-$ 101039). He noted that the valve actuator/position switch assembly and leak detection system designs are identical to the those presented in W-314's "AN Valve Pit Upgrades" design.

\section{Piping Drawings - Tom Salzano}

- Tom Salzano discussed the piping drawings included in the package, consisting of P\&IDs, jumper arrangement and section drawings, jumper assembly drawings, pit cover painting diagrams, piping layouts and details, and hydraulic diagrams (H-14-101047 through H-14-101121).

- Salzano reminded the reviewers that the 241-AX-B and 241-A-B valve pit P\&IDs $(H-14-101047$ and $H-14-101080)$ have been previously reviewed at the $30 \%$ and $60 \%$ design completion stages, and they are considered ready for approval based on the previously completed formal reviews.

- Dave Bowers asked whether the applicable W-314 drawings reflected the recently installed $W-030$ and $W-320$ installations in the $A Y / A Z$ Tank Farms. Salzano indicated that he would double-check to ensure that the new installations are identified on the W-314 piping layouts. 
- Rickenbach briefiy discussed the C2 Construction Specification that supports the 200E Upgrades constructon activities.

Shafik Rifaey (TWRS Design Authority) indicated that, in addition to the currentiy identified review team, he will provide the design package to Mark Scott for civil/structural review and to Ghurdian Singh and Mazaen Al-Wazani for electrical review.

Chuck Scaief requested that, prior to the final design review meeting, an agenda be published to allow reviewers with a specific area of interest to plan.their attaendance at the review meeting.

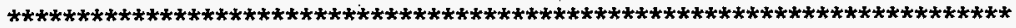

ACTION ITEN: K. A. Boes to issue agenda to review team for the design review meeting. Due date $-2 / 10 / 98$

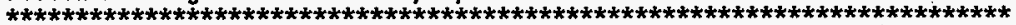

Rickenbach concluded with a discussion of questions/assumptions/issues that FDNW requests customer input on by the final design review meeting in order to finalize the 200E Upgrades design package. These issues are as follows:

1. Based on lack of definitive guidance, it has been assumed that no existing equipment in the $A X-B$ or $A-B$ pits must be retained to support saltwell pumping activities. Confirm this assumption.

2. A number of existing electrical junction boxes associated with inpit heat tracing systems are located around the outside of the A-B valve pit. The new shielding collar to be provided around the pit includes access covers for these boxes. Is this acceptable or do the temperature gages need to be relocated?

3. Are any spare conduits, in addition to those required to support the future $W-314$ Phase 2 work, required as a part of this design package?

4. The design for $A X-B$ and $A-B$ is based on shielding calculations. performed for the AN-A valve pit. Should new calculations specific to these pits be prepared to support the design?

5. The $W-314$ modifications include removal of an existing leak detection pressure switch (PS-SN-213) at the A-B valve pit. What additional modification/demolition work is required at the $242-\mathrm{A}$ Building to meet customer requirements (i.e., complete demo, or simply put into a position to ensure continued MPS system operation)?

Kirk Boes ( $W-314$ Technical Lead) closed the briefing by reminding the assembled reviewers that the technical requirements basis for the design is provided in the $W-314$ Project Development Specifications (PDSs). If any reviewer needs copies of these documents, Boes will provide. The reviewers were encouraged to contact him or any of the FDNW Design Leads if any further explanation or clarification of the design products/philosophy is needed to support their review. 
ACTION ITEM: The W-314 Design Review Team will reconvene at $7: 30$ am in the TCPC Building/4th floor conference room on Thursday, 2/12/98 (and continuing on $2 / 13 / 98$, as needed), to review/disposition the Team's final comments on the 200E Upgrades design package.

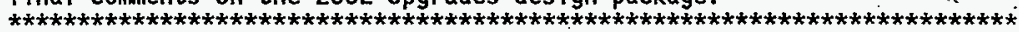

ATTACHMENT 1: 200E Upgrades Definitive Design Package Contents

ATTACHMENT 2: Definitive Design Review Kickoff Briefing Attendance List

$A-3000-480(10 / 94)$ GEF011 
Drawing List

Drawing No.

$H-14-100993$

\section{Civil/Structural}

Drawing No.

H-14-100994

$\mathrm{H}-14-10995$ Sh $1 \& 2$

$\mathrm{H}-14-10996$ Sh 122

$\mathrm{H}-14-100997$

$\mathrm{H}-14-100998$

H-14-100999

$\mathrm{H}-14-101000$

$\mathrm{H}-14-101002$ Sh 1

$\mathrm{H}-14-101002$ Sh 2

H-14-101002 Sh 3

$\mathrm{H}-14-101002 \mathrm{Sh} 4$

\section{PIPING \& VESSELS}

\section{Drawing No.}

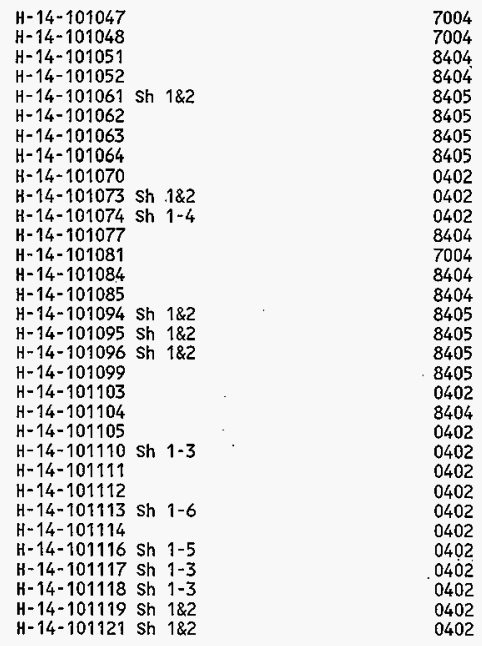

ELECTRICAL

Drawing No.

H-14-101008 Sh $1-3$

H-14-101009

$\mathrm{H}-14-101010$ sh 182

$\mathrm{H}-14-101011$

H-14-101012

H- $14-101016$

H-14-101017 sh $1 \& 2$

0402

0402

7004
7004
8404
8404
8405
8405
8405
8405
0402
0402
0402
8404
7004
8404
8404
8405
8405
8405
8405
0402
8404
0402
0402
0402
0402
0402
0402
0402
0402
0402
0402
0402
Index No. Title

0000/0101 Drawing List - Vicinity Map

\section{Index No. Title}

$0900 / 0901$

$0900 / 0901$

$0900 / 0901$

$0900 / 0901$

$0900 / 0901$

$0900 / 0901$

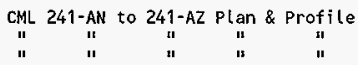

CML 241-AY to 241-AX Plan \& Profile

CML Sections \& Details - Pipe Lines

Structural Cover Blocks 241-AX-B Plan

Structüral Cover Blocks 241-A-B Plan

Structural 241-AX-B \& 241-A-B Wall Modifications

Structural pits 241-AX-B, \& A-B Plans \& Details

Structural Pits 241-AX-B, \& A-B Shielding Collar Details

structural Pits 241-AX-B, \& A-B Missile shield

Index No. Title

P\&ID Valve Pit 241-AX-B

P\&ID Miscellaneous Details

Jumper Arrangement Valve Pit 241-AX-B

Jumper Arrangement Valve $P$ it 241-AX-B section

Jumper Assembly 241-AX-B R1-R20-(O\&E)

Jumper Assembly 241-AX-B R12-(0)

Jumper Assembly 241-AX-B R16-(E)

Jumper Assembly 241-AK-B R1A-Drain

Pit Cover Painting Diagram 241-AX-B

Piping Floor Drain Seal Assembly Valve Pits 241-A-B \& AX-B Piping Valve Pit 241-AX-B Modification Details

Valve Actuator Arrangement Valve Pit 241-AK-B

P\&ID Valve Pit 241-A-B

Jumper Arrangement Valve $P$ it 241-A-B

Jumper Arrangement valve $P$ it 241-A-B section

Jumper Assembly 241-A-B R1-R2-(A)

Jumper Assembly 241-A-B R15-R19-(B)

Jumper Assembly $241-A-B(A)-(B)-(C)$

Jumper Assembly 241-A-B R16-(C)

Pit Cover Painting Diagram 241-A-B

Valve Actuator Arrangement Valve Pit 241-A-B

Piping Valve Pit 241-A-B Modification Details

Piping Plan 241-AN-B to 241-A2-02A

Piping Support Plan 3" SN-630-W9

Piping Details

Piping $P$ lan $241-A Z-02 A$ to $241-A X-B$

Piping support Plan 3" SN-632-W9

Piping Plan 241-AX-B to $241-A-B$

Piping Support Plan 3" SN-634-W9

Piping Plan 241-AX-B to 241-AY-02A

Piping support $P($ an 311 SN-633-69

Piping Hydraulic Diagram AN to A
Index No. Title

7901

7301

7301

7304

7905

7301

7301
Electrical site Plan and Details A/AN/AX/AY/AZ Tank Farms Electrical $P$ lan and Details Valve Pit 241-AX-B

Electrical Installation Details Valve Pit 241-AX-B

Electrical Panelboard Schedule DP-AX-006

Electrical Wire Run List Valve Pit 241-AX-B

Electrical Power and Control Plan 241-A Tank farm

Electrical Installation Details valve Pit $241-\mathrm{A}-\mathrm{B}$ 


\section{ELECTRICAL (continued)}

\section{Drawing No.}

\section{H-14-101018}

$\mathrm{H}-14-101019$

$H-14-101020$ sh $1-3$

H-14-101021 Sh $1-6$

H-14-101022 Sh 1-5

H-14-101023 Sh $1-3$

H-14-101024 Sh $1 \& 2$

\section{INSTRUMENTATION}

\section{Drawing No.}

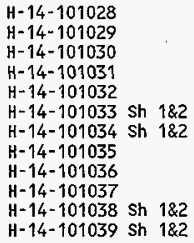

H-14-101036

$\mathrm{H}-14-101037$

H-14-101038 Sh 182

H-14-101039 Sh 182

\section{INSTRUMENTATION}

\section{Drawing No.}

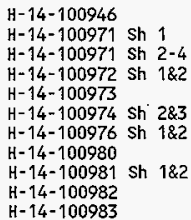

\section{CONSTRUCTION SPECIFICATION}

\section{Drawing No.}

w-314-c2

\section{Index No. Title}

7304

7905

7801

7801

7801

7801

7801

\section{Index No. Title}

5901

5901

5901

5903

5903

5903

5900

5901

5901

5901

5903

5900

\section{Index No. Title}

8404
0403
0403
0403
8405
0402
0402
590174
590174
590174
590174

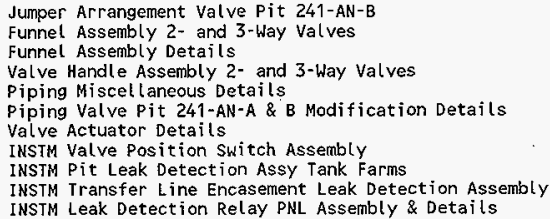

\author{
INSTM Valve Pit Plan $241-A X-8$ \\ INSTM $241-A X-B$ Valve Pit Elevation
INSTM Valve Pit Leak Detection Loop Diagram \\ ENC Low Pt Leak Det Loop Diagram \\ INSTM Valve Position Sensor Loop Diagram \\ INSTM 241-A-B Valve Pit Elevation \\ INSTM Instrument List
}

Index No. Title

Construction Specification 
HNF-2375, Rev. 0

ATTACHMENT 5

PAGE 1 of 1

ATtACHMENT ${ }^{*}$

A5-7.

KICK-OFF MEETING FOR FINAL REVIEW 1/29/98. W-314"ZOOE/W UPGRADES"

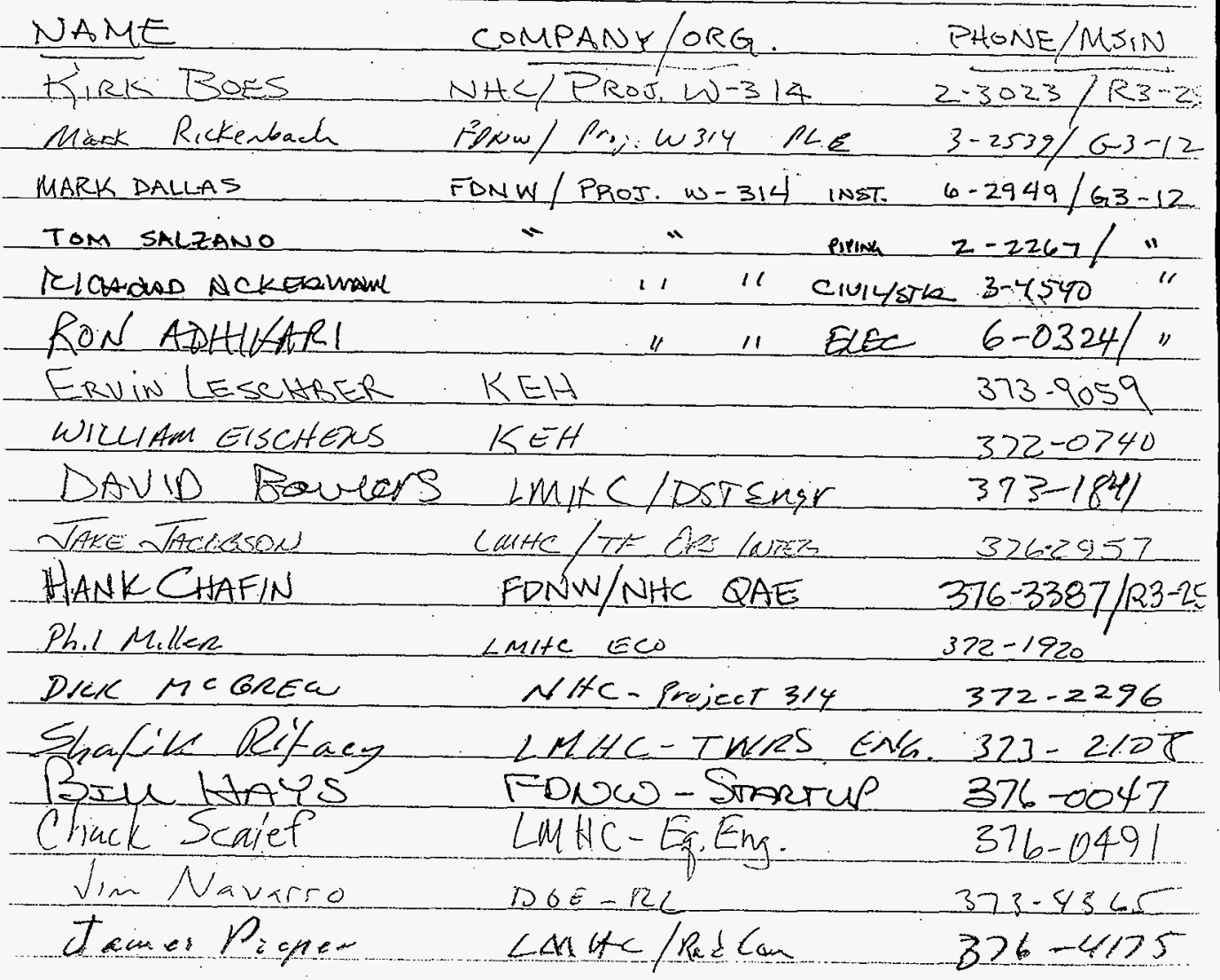


Subject: FINAL DESIGN REVIEW MEETING - $W-314$ 200E UPGRADES

T0: Distribution

FROM: Kirk Boes

\section{BUILDING}

CHAIRMAN Kirk Boes

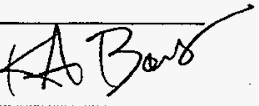

Department-0perationComponent

TWRS/Project $W-314$
Area
Shift Date of Heeting
$2 / 12 / 98-2 / 13 / 98$

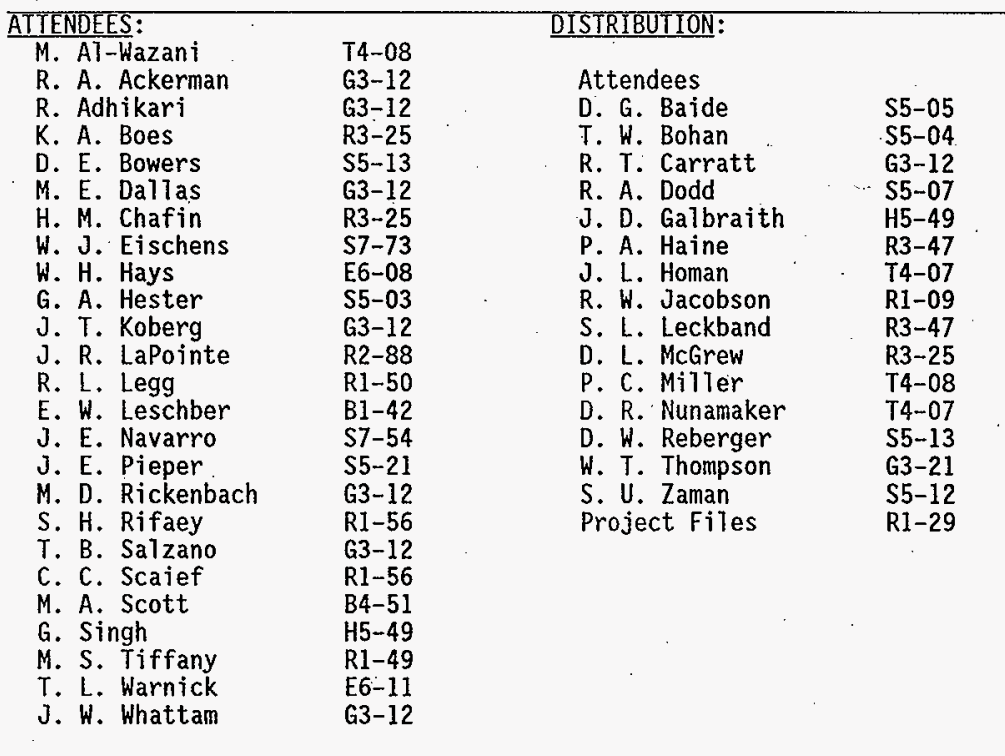

These minutes document the discussions and actions from the final definitive design review meeting for Project W-314's "200E Upgrades" design package. Copies of the design package (consisting of 136 drawings and the $\mathrm{C}-2$ construction specification) and specific instructions for the final review were provided to the $\mathrm{W}-314$ Design Review Team (DRT) at the final design review kick-off briefing conducted on $1 / 29 / 98$. Participants during the two-day review are identified in the attachment.

The assembled personne] reviewed each of the design drawings one by one. Any comments or questions related to a particular drawing were discussed and, in most cases, a disposition was assigned to the comment during the meeting (e.g., comment accepted, comment not accepted, etc.). Comments that were dispositioned as "accepted" or which were determined to need further evaluation by the Design Team were recorded on a consolidated Review Comment Record (RCR) form. This RCR (designated as RCR \#200E002) represents the formal comments of the $\mathrm{H}-314$ DRT. Likewise, the $\mathrm{C}-2$ 
construction specification was also reviewed page by page and the team's ATTACHMENT 5

comments were dispositioned and recorded accordingly. Closure of theRCR

(indicated by approval signatures from each of the pareticipating review organizations) will signify the DRT's acceptance of the stated comment dispositions, and provides the basis for final approval of the design package.

Decisions regarding several key questions/issues raised by the FDNW Design Team during the $1 / 29 / 98$ briefing are identified below:

1. Based on lack of definitive guidance, it has been assumed that no existing equipment in the $A X-B$ or $A-B$ pits must be retained to support saltwell pumping activities. Confirm this assumption.

Decision: All existing jumpers in the valve pits will be removed and disposed of by the project.

2. A number of existing electrical junction boxes associated with inpit heat tracing systems are located around the outside of the A-B valve pit. The new shielding collar to be provided around the pit includes access covers for these boxes. Is this acceptable or do the temperature gages need to be relocated?

Decision: The project will reattach the loose $j$-boxes to the outside of the shiled collar during construction. No further modifications to these items is planned.

3. Are any spare conduits, in addition to those required to support the future $W-314$ Phase 2 work, required as a part of this design package?

Decision: No spare conduits, besides those required for $\mathrm{W}-314-$ related activities, will be provided.

4. The design for $A X-B$ and $A-B$ is based on shielding calculations performed for the AN-A valve pit. Should new calculations specific to these pits be prepared to support the design?

Decision: New shielding calculations should be provided to substantiate the need for the collars.

5. The $\mathrm{H}-314$ modifications include removal of an existing leak detection pressure switch (PS-SN-213) at the A-B valve pit. What additional modification/demolition work is required at the $242-A$ Building to meet customer requirements (i.e., complete demo, or simply put into a position to ensure continued MPS system operation)?

Decision: Isolation of the existing pressure switch for the SN$200 / 213$ encasement leak detector at the 242-A facility should not be included in the 200E Upgrades design package (per Dave Bowers). The work at 242-A needs to be performed via ECN against the existing 242-A Evaporator drawings. The basic. work needing to be done involves isolating the instrument air supply to the pressure switch/transfer line encasement and electrically disconnecting or bypassing the switch from the shutdown/alarm relays. Software changes in the Evaporator MCS will also be required. Tom Galioto is the POC for coordinating Evaporator facility changes, and Mac Teats is the POC for software changes. 
The W-314 Technical Lead (Kirk Boes) will follow up with each review team member as needed to complete the formal comment resolution and

close-out process. Comments will be incorporated into the $200 \mathrm{E}$ Upgrades design media by FDNW's Design Team as indicated on the official RCR. Upon completion of this activity and verification that all comments were properiy incorporated, the 200E Upgrades design package will be approved and released for construction. Anyone needing further information regarding this process should contact Kirk Boes at 372-3023.

In addition to the W-314 DRT, the U.S. Department of Energy, Richland operations office (RL) participated in the review of the 200E Upgrades design package. Comments from the RL independent reviewers (Erv Leschber and Bill Eischens) are included in the official RCR along with the comments of the DRT.

ATTACHMENT: Attendance 7 ists from $2 / 12$ and $2 / 13$ review meetings 
$2112 \times 198$

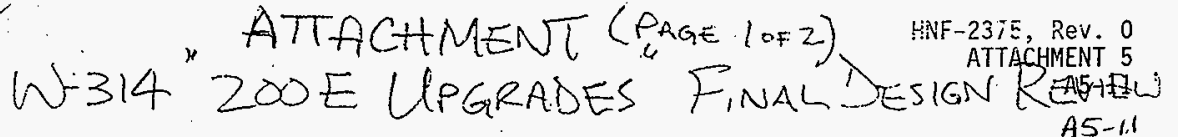

NAME

COMPANY JorG.

MSIN/PHONE

KIRK BOES NHS/PRJ W-314

Mark Rickerbach Finw/ w3/4

$R 3-25 / 372-3023$

Jorty Koiserg Fan /PM

$0-3-12 / 373-2579$

Chuak Scaief

LMHC/Eg. Eng:

$-6-3-12 / 373-2576$

Terry le 2arixck FDNW/startup

$R 1-56,376-0491$

Treta Ravencraft imAC/OE. S5-04/323-9275

Row Ley

LmHClossing

$329-4123$

Ihere tyester kinte/Pic

$323-3236$

RLCAND ACHERman

Foric

$373-4540$

Dick CarratT

FDNW

$372-3726 / 63-12$

mark E Dallas

FDNW

$376-2949 / 63-12$

Jeff Whattem

Fond

$336-6401 / 63-12$

GURDHIAN SINGGA

NitC

RON ADHULAAI

EDNW

376.3129

ERV Laschber KEH $373-9059$

TOM SALZANO FDNW 372-2267

BILL EISCHLAS KEH $\quad 372-0740$

HANKCHAFIN

FDNW

$R 3-25 / 376-338.67$

DAvID Bowers

LMSE

$55-13 / 3.73-184)$

Hether S Totong.

DESH

$R /-49 / 373-2148$

mazen Aluaremi

LMHC

$24-07,1372-3569$

Shafik Rifacy

LMAC

$21-56 / 373-2108$

HAMESR. LA Ponve

Lattc

$R 2-88+373-7244$

Tamer Preper LAHC S5-21 3764175

Maxk Soti SEsc BA-51/376-5152 
$2 / 13198$

ATTACHMENT (PAGE Z OF 2) HNF-2375, Rev. 0

ZOOE UPGradeES FINAL. DesIGN ReVIEW

A5-12

NAME

COMPANY/ORQ

MSIN/PHONE

Kirk Boes

DAUID Bowers

Join KoBrose

Hank Corafin

BILL EISCHEOS

GURDHION SINGH

Jim Navarro

ERV LESCHBER

Terry Uarnick

Richors devermonu

RON ADHIKAR.

Jerf Whattam

Shafik Rifacy

Kow hegs

QHFEN AESTER

R.T. Cancit

MARK E. DALLAS

TOM SALZANO

Sim LApointe ume cuc

prark Reckestach Fprow

EDNW/PM

FDNW/QAE

$k \in H$

NEIC

DUE-RL

KEH

Fones

CDNW

EONW

$\angle M A C$

$\operatorname{Lin} A C$

EONG

FDNW

“
NHS/Proj.w-314

LMHE/DSTEng

FDNW/Stantup

$\operatorname{Lm}(x$

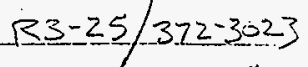

$55=13 / 373-644$

$63-12 / 373-2576$

$R 3-25 / 376-3307$

$3>2-0740$

$376-3129$

$323-4365$

373.9059

$376-2365$

$373-4540$

$326-0324$

$03-12 / 376-6901$

$373-208$

$373-4223$

$373-323$

$372-3726$

$376-2949$

$372-2267$

$22-88 \quad 373-2744$

$63-12 / 3-2539$ 


\begin{tabular}{|c|c|}
\hline $\begin{array}{l}\text { 1. Date } \\
2 / 12 / 98 \\
\end{array}$ & $\begin{array}{r}\text { 2. Review No. } \\
200 E-002 \\
\end{array}$ \\
\hline $\begin{array}{r}\text { 3. Project No. } \\
\mathrm{W}-314\end{array}$ & 4. Page \\
\hline
\end{tabular}

\section{Document Number (s)/Title (s) \\ Project W-314 - Phase 1, "200E Upgrades"} Definitive Design Drawings and Specifications 10. Agreement with indicateg comment disposition(s)

$$
\text { Project Developpent (W-314 Engineering Lead) }
$$

K) and 5 Somers

DST Engineering (H-314 Design Authority)

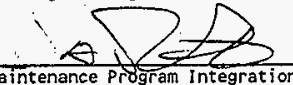

Maintenance Program Integration

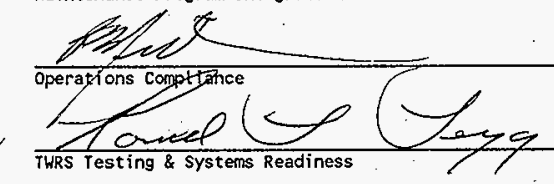

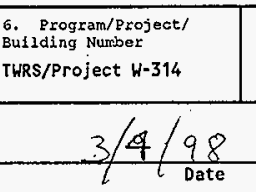

7. Revlewer

See belon

$3 / 9 / 98$

$3 / 9 / 98$

$3 / 5 / 98$

Date

TWRS Testing \& Systems Readiness

8. Organization/Group
See below

9. Iocation/Phone

Gimanarer

TWRS Safety

Safety

$\frac{1}{\text { Process Development }}$

Rarf Engineering/lecknical support

Hoinm

$\checkmark$ Project Developme flectrica!)

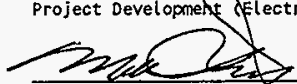

SESC Mechanical/civil

L

RL Independent Review

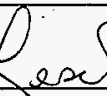

$\operatorname{coc} x$

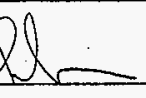

$\frac{7-9-98}{\text { Date }}$

$\frac{3-9-98^{2}}{\text { Date }}$

$3-10-48$

$3 / 9 / 58$

$3 / 9 / 98$

$3 / 13 / 98$

$3 / 10 / 0.8$ siit $K$ Pn the

siit $K$ ph th

Project Development (H-314 Systems Engineering Lead)

$3 \cdot 9-98$

Aleme Afestes

operations \& Project Support

$\frac{3-10-98}{\text { Date }}$

teflection

operztions integration

$\frac{23.3 / 0 / 98}{\text { Date }}$

Haukm. Chabin

adalijes Assurance i

$c 2 \mathrm{~A}=\mathrm{a}$

startup (R-314 Startup Lead

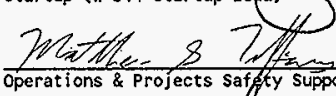

$3 / 10 / 98$

Date

$3-9.98$

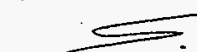

Equipnent Engineering ?

$\frac{\text { C.C. Scunf II }}{\text { Equipment Engineer ing }}$

$3-10-98$

SST Engineering

Date

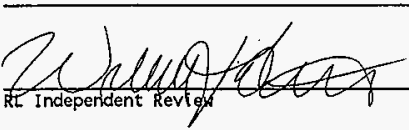

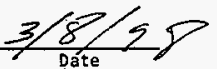

$3 / 10 / 90$ Date? 


\begin{tabular}{|l|l|}
\hline $\begin{array}{l}\text { 1. Date } \\
2 / 12 / 98\end{array}$ & $\begin{array}{l}\text { 2. Review No. } \\
200 \mathrm{E}-002\end{array}$ \\
\hline $\begin{array}{l}\text { 3. Project No. } \\
\text { W-314 }\end{array}$ & 4. Page \\
\hline
\end{tabular}

\begin{tabular}{|c|c|c|c|c|}
\hline $\begin{array}{l}12 . \\
\text { Item }\end{array}$ & $\begin{array}{l}\text { 13. Coment(s)/Discrepancy(s) (Provide technical justification for the comment } \\
\text { and detailed recommendation of the action required to correct/ resolve the } \\
\text { discrepancy/problem indicated.) }\end{array}$ & $\begin{array}{l}14 . \\
\text { Hoid } \\
\text { Point }\end{array}$ & 15. Disposition (Provide justification if NOT accepted.) & $\begin{array}{l}16 . \\
\text { Status }\end{array}$ \\
\hline 1 & $\begin{array}{l}\text { H-14-101008, sht. } 1 \text { : Delete spare (non-W-314) conduits - } \\
\text { there is no requirement to provide these items. (Boes) }\end{array}$ & & $\begin{array}{l}\text { (cp) Accepted. All spare conduit (Non-W-314) } \\
\text { deleted. }\end{array}$ & \\
\hline 2 & $\begin{array}{l}\text { H-14-101008. sht. 1: Field routed conduits will need to be } \\
\text { as-built on final project drawings. (Bowers) }\end{array}$ & & Noted. & \\
\hline 3 & $\begin{array}{l}\mathrm{H}-14-101008 \text {, sht. } 1 \text { : Specify (in C-spec) sealing material } \\
\text { referred to in Note } 9 \text {. (Singh) }\end{array}$ & & $\begin{array}{l}\text { Accepted. Note revised to cap conduits instead of } \\
\text { sealing. }\end{array}$ & \\
\hline 4 & H-14-101008, sht. 2 - no comments & & N/A & \\
\hline 5 & $\begin{array}{l}\mathrm{H}-14-101008 \text {, sht. } 3 \text { - no comments (sheet maybe deleted based } \\
\text { on comment } 1 \text { ) }\end{array}$ & & N/A & . \\
\hline 6 & $\begin{array}{l}\text { H-14-101009: Reorientate Section A shown in Detail I to match } \\
\text { the Elevation A drawing. (Singh) }\end{array}$ & & Accepted. Re-orientated section A. & \\
\hline 7 & $\begin{array}{l}\text { H-14-101009: Correct mislabeling of conduits } \mathrm{C} 3 \text { and } \mathrm{C} 8 \\
\text { (Singh) }\end{array}$ & & Accepted. Switched labeling of $\mathrm{C} 3$ and $\mathrm{C} 8$. & . \\
\hline 8 & $\begin{array}{l}\text { H-14-101009: De lete conduit shown extending north from } \\
\text { TBX101. (Singh) }\end{array}$ & & Accepted. Deleted conduit. & \\
\hline 9 & $\begin{array}{l}\text { H-14-101009: Clarify tag numbers shown on leak detection } \\
\text { relay cabinets (complete tag numbers). (Singh) }\end{array}$ & & (cp) Accepted. Put in whole tag number. & \\
\hline 10 & $\begin{array}{l}\text { H-14-101010, sht. 1: Correct sizing of penetration show for } \\
\text { gas sampling port. (Boes) }\end{array}$ & & $\begin{array}{l}\text { Accepted. Gas sampling port size reduced to reflect } \\
1 \text {-inch penetration. }\end{array}$ & \\
\hline 11 & $\begin{array}{l}\text { H-14-101010, sht. 1: Check reference drawing identified for } \\
\text { anchoring in Elevations B and C. (Chafin) }\end{array}$ & & Accepted. Changed reference to $\mathrm{H}-14-101002$. & \\
\hline 12 & $\begin{array}{l}\text { H-14-101010, sht. } 2: \text { In detai] shown for cordset schedule. } \\
\text { clarify that note referencing " } 90 \text { degrees" is for temperature } \\
\text { (Centigrade). (Singh) }\end{array}$ & & $\begin{array}{l}\text { Accepted. Added " } \mathrm{C} \text { " to the note to indicate degrees } \\
\text { centigrade. }\end{array}$ & \\
\hline 13 & $\begin{array}{l}\text { H-14-101010, sht. 2: Evaluate need for shielded wire. } \\
\text { (Singh) }\end{array}$ & & $\begin{array}{l}\text { (cp) Accepted. Will use shield for whole run. Cord } \\
\text { set changed to include shield. }\end{array}$ & \\
\hline \multicolumn{5}{|c|}{ A-6400-090.1 (03/92) WEF011 } \\
\hline
\end{tabular}




\section{REVIEW COMMENT RECORD (RCR)}

\begin{tabular}{|c|l|}
\hline $\begin{array}{l}\text { 1. Date } \\
2 / 12 / 98\end{array}$ & $\begin{array}{l}\text { 2. Review No. } \\
200 \mathrm{E}-002\end{array}$ \\
\hline $\begin{array}{c}\text { 3. Project No. } \\
\text { W-314 }\end{array}$ & 4. Page \\
\hline
\end{tabular}

\begin{tabular}{|c|c|c|c|c|}
\hline $\begin{array}{l}12 . \\
\text { Item }\end{array}$ & $\begin{array}{l}\text { 13. Corment(s)/0iscrepancy(s) (Provide technical justification for the comment } \\
\text { and detailed recommendation of the action required to correct/ resolve the } \\
\text { discrepancy/problem indicated.) }\end{array}$ & $\begin{array}{l}14 . \\
\text { Hoid } \\
\text { Point }\end{array}$ & 15. Disposition (Provide justification if NOT accepted.) & $\begin{array}{l}16 . \\
\text { Status }\end{array}$ \\
\hline 14 & $\begin{array}{l}\text { H-14-101010, sht. 2: Clarify pin numbering matchups on } \\
\text { cordset connections (additional details and/or notes). } \\
\text { (Singh) }\end{array}$ & & $\begin{array}{l}\text { (cp) Accepted: Revised notes } 12 \text { and } 13 \text { to clarify } \\
\text { pin matchups. }\end{array}$ & \\
\hline 15 & $\begin{array}{l}\text { General comment: Need to obtain vendor submittal data for all } \\
\text { procured items. (Bowers) }\end{array}$ & & $\begin{array}{l}\text { D. Bowers will identify what items require vendor } \\
\text { info. }\end{array}$ & \\
\hline 16 & H-14-101011 - no comments & & N/A & \\
\hline 17 & $\begin{array}{l}\text { H-14-101012: Verify conduit routings shown for wire run - see } \\
\text { comment \#7. (A7-Wazani) }\end{array}$ & & Verified. Wire run list is correct as shown. & \\
\hline 18 & $\begin{array}{l}\text { H-14-101009, : Add TBX numbers (consistent with wire run } \\
\text { list) to enlarged plan for clarification. (Al-Wazani) }\end{array}$ & & (cp) Accepted. Added TBX numbers. & . \\
\hline 19 & $\begin{array}{l}\text { H-14-101012: Evaluate using terminal strips for wire runs } 4 \text {, } \\
5 \text { and } 6 . \text { (Singh) }\end{array}$ & & $\begin{array}{l}\text { (cp) Not accepted. No splicing involved with signal } \\
\text { wire (continuous wire) so there is no need for } \\
\text { terminal strips. }\end{array}$ & \\
\hline 20 & $\begin{array}{l}\text { H-14-101012: Add note to clarify "WT" prefixes to tag } \\
\text { numbers. (A7-Wazani) }\end{array}$ & & $\begin{array}{l}\text { (cp) Accepted for LDE only. Others are explained in } \\
\text { Note } 3 \text {. }\end{array}$ & \\
\hline 21 & $\begin{array}{l}\text { H-14-101016: In Detail 2, correct mislabeling of conduits } \\
\text { (one conduit labeled as both C3 and C41). (Al-Wazani) }\end{array}$ & & Accepted. Additional conduit label added. & \\
\hline 22 & $\begin{array}{l}\text { H-14-101010; sht. 1: Reorientate Section A line to match } \\
\text { elevation A view. }\end{array}$ & & Accepted. Section "A" re-orientated. & \\
\hline 23 & H-14-101016: Delete Note 4.B. (Chafin) & & Accepted. Note 4.B deleted. & \\
\hline 24 & $\begin{array}{l}\text { H-14-101016: Identify conduit CA1, as appropriate, on } \\
\text { Elevation A. }\end{array}$ & & Accepted. Identified conduit C41. & \\
\hline 25 & $\begin{array}{l}\text { H-14-101017. sht. 1: Correct Section A line shown on Plan } \\
\text { View to be consistent with section view. }\end{array}$ & & Accepted. Corrected section A lines. & \\
\hline 26 & $\begin{array}{l}\text { H-14-101017, sht. 2: Incorporate applicable comments from } \mathrm{H} \text { - } \\
\text { 14-101010, sht. 2. }\end{array}$ & & Accepted. See items 10 through 14 , and 22 . & \\
\hline 27 & Not used. & & N/A & \\
\hline 28 & H-14-101018: No comments & & $\mathrm{N} / \mathrm{A}$ & \\
\hline
\end{tabular}




\begin{tabular}{|c|l|}
\hline $\begin{array}{l}\text { 1. Date } \\
2 / 12 / 98\end{array}$ & $\begin{array}{l}\text { 2. } \\
200 \mathrm{E}-002\end{array}$ \\
\hline $\begin{array}{c}\text { 3. Project No. } \\
\text { W-314 }\end{array}$ & $\begin{array}{l}\text { 4. Page } \\
\text { of } 33\end{array}$ \\
\hline
\end{tabular}

\begin{tabular}{|c|c|c|c|c|}
\hline $\begin{array}{l}12 . \\
\text { Item }\end{array}$ & $\begin{array}{l}\text { 13. Comment(s)/Discrepancy(s) (Provide technical justification for the corment } \\
\text { and detailed recomendation of the action required to correct/ resolve the } \\
\text { discrepancy/problem indicated.) }\end{array}$ & $\begin{array}{l}14 . \\
\text { Hold } \\
\text { Point }\end{array}$ & 15. Disposition (Provide justification if NOT accepted.) & $\begin{array}{l}16 . \\
\text { Status }\end{array}$ \\
\hline 29 & H-14-101019: same comments as for H-14-101012. & & (cp) Accepted. See items 17, 19, and 20. & \\
\hline 30 & $\begin{array}{l}\text { H-14-101020. sht. 1: Concerning Note } 3 \text {, ensure that any } \\
\text { anodes are as-built within plus/minus one foot. (Bowers) }\end{array}$ & & $\begin{array}{l}\text { (cp) Accepted. Revised Note } 3 \text { to àdd "Record actual } \\
\text { installed locations if other than shown". }\end{array}$ & \\
\hline 31 & $\begin{array}{l}\text { H-14-101020, sht. } 1 \text { : Concerning Note } 11 \text {, verify acceptability } \\
\text { of abandoning anodes in place. In addition, final as-builting } \\
\text { sha } 11 \text { reflect any existing anodes which are removed. (Bowers) }\end{array}$ & & $\begin{array}{l}\text { Checked with LMHC's Operations Compliance } \\
\text { organization (Phil Miller) and it is acceptable to } \\
\text { abandon them. } \\
\text { Noted that final as-built drawings should reflect } \\
\text { anodes which are removed. }\end{array}$ & \\
\hline 32 & $\begin{array}{l}H-14-101020 \text {, sht. 1: Revise jumper shown connecting SN-630 } \\
\text { and SN-260 lines to indicate "existing" (to be installed as } \\
\text { part of AN Valve Pit Upgrades construction package). (Boes) }\end{array}$ & & $\begin{array}{l}\text { (cp) Accepted. Revised connections to display as } \\
\text { "existing". }\end{array}$ & \\
\hline 33 & $\begin{array}{l}\text { H-14-101020, sht. I: Clarify method of connection of } \mathrm{CP} \text { to } \\
\text { pipe encasement. (Singh) }\end{array}$ & & $\begin{array}{l}\text { (cp) Accepted. Revised drawing to show line } \\
\text { representing the negative return cable connecting to } \\
\text { encasement symbol. }\end{array}$ & \\
\hline 34 & H-14-101020, sht. 2 - no comment & & N/A & \\
\hline 35 & $\begin{array}{l}\mathrm{H}-14-101020 \text {, sht. } 3 \text { : Existing test station } T(11-203) \text { is to be } \\
\text { relocated. Clarify where existing station is moved from } \\
\text { (typical for all relocated test stations). (Bowers) }\end{array}$ & & $\begin{array}{l}\text { (cp) Not accepted. Existing test station locations } \\
\text { and relocation, as necessary, are shown on civil } \\
\text { drawings. This drawing shows connections to the pipe } \\
\text { end only. }\end{array}$ & \\
\hline 36 & $\begin{array}{l}\text { H-14-101020, sht. 3, editorial - clean up labeling in zone D4. } \\
\text { (Chafin) }\end{array}$ & & Accepted. Made lâbeling more readable. & \\
\hline 37 & $\begin{array}{l}\text { H-14-101021, sht I: See comments concerning general notes ( } 30 \\
\text { and } 31 \text { ). }\end{array}$ & & Noted. & \\
\hline 38 & $\begin{array}{l}\text { H-14-101021, sht. 1: Verify that test station number T(11- } \\
\text { 206) has not already been used. (Scaief/Bowman) }\end{array}$ & & $\begin{array}{l}\text { Accepted. New number assigned. } T(11-208) \text { and shown } \\
\text { on the drawing. }\end{array}$ & \\
\hline 39 & $\begin{array}{l}\text { H-14-101021, sht. 2: Add labels for } 2 \text { lines covered by test } \\
\text { station T97-62). (Scaief/Bowman) }\end{array}$ & & Accepted. Added line numbers (PW-4805 and PW-4507). & \\
\hline 40 & H-14-101021, shts, $3,4,5$, and 6 - no comment & & N/A & \\
\hline
\end{tabular}




\section{REVIEW COMMENT RECORD (RCR)}

\begin{tabular}{|c|c|}
\hline $\begin{array}{l}\text { 1. Date } \\
2 / 12 / 98\end{array}$ & $\begin{array}{l}\text { 2: Review No. } \\
200 \mathrm{E}-002\end{array}$ \\
\hline $\begin{array}{r}\text { 3. Project } \text { No. } \\
\text { W-314 }\end{array}$ & 4. Page \\
\hline
\end{tabular}

\begin{tabular}{|c|c|c|c|c|}
\hline $\begin{array}{l}12 . \\
\text { Item }\end{array}$ & $\begin{array}{l}\text { 13. Comment(s)/Discrepancy(s) (Provide technical justification for the comment } \\
\text { and detailed recommendation of the action required to correct/ resolve the } \\
\text { discrepancy/problem indicated.) }\end{array}$ & $\begin{array}{l}\text { 14. } \\
\text { Hold } \\
\text { Point }\end{array}$ & 15. Disposition (Provide justification if NOT accepted.) & $\begin{array}{l}16 . \\
\text { Status }\end{array}$ \\
\hline 41 & $\begin{array}{l}\text { H-14-101022, sht. 1: Clean up labeling associated with new } \\
\text { test stations. Same comment for sht. } 4 \text { and } 5 \text {. (Boes) }\end{array}$ & & Accepted. Labeling made more readable. & \\
\hline 42 & $\begin{array}{l}\text { H-14-101022, sht. I: Apply any applicable comments for } \\
\text { previous CP drawings. (Bowers) }\end{array}$ & & Accepted. & \\
\hline 43 & $\begin{array}{l}\text { H-14-101022, sht. 2: Verify correct labeling of anodes and } \\
\text { confirm consistency. between plan and table. (Scaief/Bowman) }\end{array}$ & & $\begin{array}{l}\text { Verified. Changed anode numbers } A(14-50) \text { ( } 3 \text { places) } \\
\text { to } A(14-56) \text {. } A(14-57) \text {, and } A(14-58) \text { and corrected } \\
\text { anode location table. }\end{array}$ & \\
\hline 44 & H-14-101022, sht. 3 - no comment & & N/A & \\
\hline 45 & $\begin{array}{l}\text { H-14-101022, sht. 4: Verify sufficient spare space is } \\
\text { available for } W-314 \text { 's use in existing conduit for ADB(15-2). } \\
\text { Evaluate need for new ADB. (Scaief/Bowman) }\end{array}$ & & $\begin{array}{l}\text { Verified that enough space is available. Conduit run } \\
\text { is short (less than } 3 \mathrm{ft} \text {.). }\end{array}$ & \\
\hline 46 & H-14-101022, sht. 5 - no comment & & $\mathrm{N} / \mathrm{A}$ & \\
\hline 47 & $\begin{array}{l}\text { H-14-101023, sht 1: See comments concerning general notes for } \\
\text { previous CP drawings. }\end{array}$ & & Accepted. & \\
\hline 48 & $\begin{array}{l}\text { General comment: Verify that recent } \mathrm{CP} \text { installations } \\
\text { associated with other projects have been considered in this } \\
\text { design. (Bowers). }\end{array}$ & & $\begin{array}{l}\text { (cp) Accepted. Evaluated and design has no adverse } \\
\text { effects on CP System in field. }\end{array}$ & \\
\hline 49 & $\begin{array}{l}\text { H-14-101023, sht. I: Add appropriate north coordinates to } \\
\text { this drawing for reference (Al-wazani) }\end{array}$ & & (cp) Accepted. Added north coordinate to pit AX-B. & \\
\hline 50 & $\mathrm{H}-14-101023$. sht 2 and 3 - no comment & & $\mathrm{N} / \mathrm{A}$ & \\
\hline 51 & H-14-101024, sht: 1 and 2 - no comment & & N/A & \\
\hline 52 & H-14-101028 - no comment & & N/A & \\
\hline 53 & $\begin{array}{l}\text { H-14-101029: Add triangle symbol (see Note 3) to Legend at } \\
\text { top of drawing. (Bowers) }\end{array}$ & & $\begin{array}{l}\text { (cp) Not accepted. Definition of this symbol is } \\
\text { covered by Note } 3 .\end{array}$ & \\
\hline 54 & $\begin{array}{l}\mathrm{H}-14-101029: \text { Verify correct labeling of } \mathrm{HH}-01 \text { in Zone } \mathrm{F} 6 . \\
\text { (Singh) }\end{array}$ & & Verified, correct as shown. & \\
\hline-55 & $\begin{array}{l}\text { H-14-101029: Evaluate providing installation details for } \\
\text { terminal box (see Note 4). (Leschber) }\end{array}$ & & $\begin{array}{l}\text { (cp) Accepted. See installation details on drawing } \\
\mathrm{H}-14-101009 \text {, note } 4 \text {. }\end{array}$ & \\
\hline
\end{tabular}




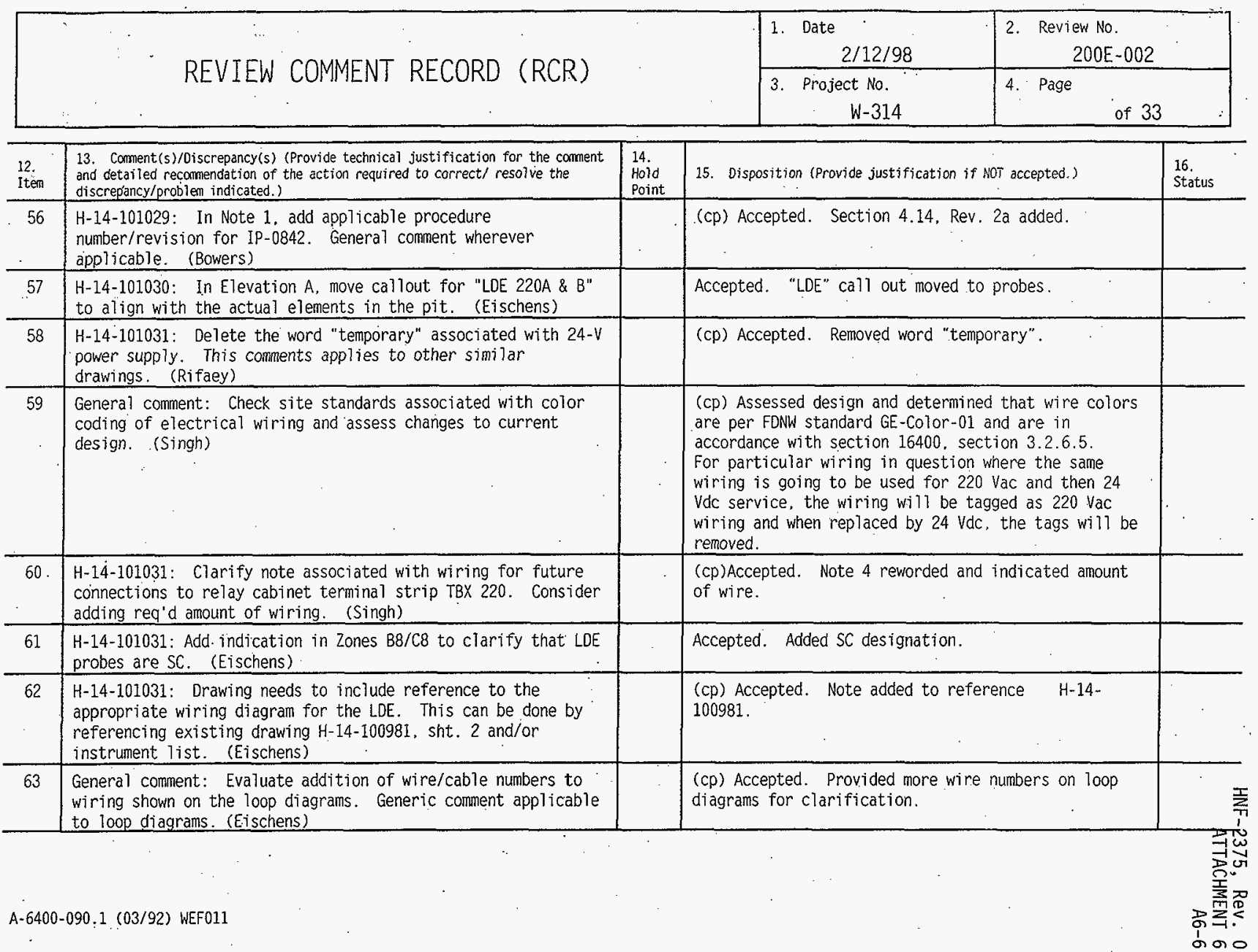




\section{REVIEW COMMENT RECORD (RCR)}

\begin{tabular}{|l|l|}
\hline $\begin{array}{l}\text { 1. Date } \\
2 / 12 / 98\end{array}$ & $\begin{array}{l}\text { 2. Review No. } \\
200 \mathrm{E}-002\end{array}$ \\
\hline $\begin{array}{c}\text { 3. Project No. } \\
\text { W-314 }\end{array}$ & 4. Page \\
\hline
\end{tabular}

\begin{tabular}{|c|c|c|c|c|}
\hline $\begin{array}{l}12 . \\
\text { Item } \\
\end{array}$ & $\begin{array}{l}\text { 13. Comment(s)/Discrepancy(s) (Provide technical justification for the comnent } \\
\text { and detailed recommendation of the action required to correct/ resolve the } \\
\text { discrepancy/problem indicated.) }\end{array}$ & $\begin{array}{l}\text { 14. } \\
\text { Hold } \\
\text { Point }\end{array}$ & 15. Disposition (Provide justification if NOT accepted.) & $\begin{array}{l}16 . \\
\text { Status }\end{array}$ \\
\hline 64 & $\begin{array}{l}\text { General comment: Evaluate adding wiring diagrams for all } \\
\text { electrical installations. (Leschber) }\end{array}$ & & $\begin{array}{l}\text { (cp) Accepted. Added wiring diagrams for AX241-WT- } \\
\text { TBX-101, -PNL-222, -PNL-220, }- \text {-W314-07, -W314- } \\
\text { 03. A241-WT-TBX-101, - } \\
\text { W314-05, -W314-03, -W314-02 as shown on drawings } \\
\text { H-14-101040. }\end{array}$ & \\
\hline 65 & $\begin{array}{l}\text { H-14-101032: Incorporate any applicable comments from drawing } \\
\text { 31. (Bowers) }\end{array}$ & & (сp) Accepted. See items 58, 60, 61, and 62 . & \\
\hline 66 & $\begin{array}{l}\text { H-14-101032; Add reference for method of accomplishing the } \\
\text { encasement LDE electrical ground. (Singh) }\end{array}$ & & $\begin{array}{l}\text { (cp) Accepted. Added new notes for reference drawing } \\
\mathrm{H}-14-100982 \text {. }\end{array}$ & \\
\hline 67 & $\begin{array}{l}\text { General comment: Evaluate adding terminal strip numbers to } \\
\text { loop diagrams. (Singh) }\end{array}$ & & $\begin{array}{l}\text { (cp) Accepted. Added TB-G number on } \mathrm{H}-14- \\
101033 \text { and }-101038 \text {. Other loops are sufficient as } \\
\text { is. }\end{array}$ & \\
\hline 68 & Not used & & $N / A$ & \\
\hline 69 & $\begin{array}{l}\text { H-101033: Change "triangle" symbol to another symbol to avoid } \\
\text { confusion with the symbol used to designate SC on other } \\
\text { drawings. (Bowers) }\end{array}$ & & $\begin{array}{l}\text { (cp) Accepted. Triangle changed to a different } \\
\text { symbol. }\end{array}$ & \\
\hline 70 . & $\begin{array}{l}\text { H-14-101034, sht. } 1 \text { and 2: Repiace "*" in table with } \\
\text { appropriate drawing reference. (Bowers) }\end{array}$ & & (cp) Accepted. 'Drawing references placed in table. & \\
\hline 71. & $\begin{array}{l}\text { H-14-101035: No comments other than already discussed for } \mathrm{H} \text { - } \\
\text { 14-101029. }\end{array}$ & & Accepted. See items $53-56$. & \\
\hline 72 & H-14-101036: See comments for H-14-101030. & & Accepted. See item 57. & \\
\hline 73 & $\begin{array}{l}\text { H-14-101037: Add references for existing wire runs shown in } \\
\text { Zone D2. (Bowers) }\end{array}$ & & $\begin{array}{l}\text { (cp) Accepted. Added reference to Spec. B-102- } \\
\text { C1. }\end{array}$ & \\
\hline 74 & H-14-101038: See comments for H-14-101033. & & (cp) Accepted. See item 69. & \\
\hline 75 & H-14-101039: See comments for H-14-101034. & & (cp) Accepted. See item 70 . & 䒺 \\
\hline 76 & $\begin{array}{l}\mathrm{H}-14-100993 \text { : Correct title shown on drawing list for } \mathrm{H}-14 \text { - } \\
\text { 100997. (Chafin). }\end{array}$ & & Accepted. Drawing title corrected on drawing list. & \\
\hline
\end{tabular}




\section{REVIEW COMMENT RECORD (RCR)}

\begin{tabular}{|l|l|}
\hline $\begin{array}{l}\text { 1. Date } \\
2 / 12 / 98\end{array}$ & $\begin{array}{r}\text { 2. } \begin{array}{r}\text { Review No. } \\
200 \mathrm{E}-002 .\end{array} \\
\hline \begin{array}{c}\text { Project No. } \\
\text { W-314 }\end{array}\end{array}$ \\
\hline
\end{tabular}

\begin{tabular}{|c|c|c|c|c|}
\hline $\begin{array}{l}12 . \\
\text { Item }\end{array}$ & $\begin{array}{l}\text { 13. Comment(s)/Oiscrepancy(s) (Provide technical justification for the corment } \\
\text { and detailed recomendation of the action required to correct/ resolve the } \\
\text { discrepancy/problem indicated.) }\end{array}$ & $\begin{array}{l}14 . \\
\text { Hoid } \\
\text { Point }\end{array}$ & 15. Disposition (Provide justification if NOr accepted.) & $\begin{array}{l}16 . \\
\text { Status } \\
\end{array}$ \\
\hline 77 & $\begin{array}{l}\text { H-14-100994: Deiete "SLOPE TBD" note on portion of new line } \\
\text { to be completed during AZ design package. (Bowers). }\end{array}$ & & (cp) Accepted. Deleted "Slope TBD". & \\
\hline 78 & $\begin{array}{l}\text { General comment: (1) On the civil plans/profile drawings, } \\
\text { evaluate adding identification of all existing underground } \\
\text { lines known to cross the new transfer lines. (2) Clarify in } \\
\text { notes that the profiles only address underground piping. } \\
\text { ductbanks. etc. (i.e.. no electrical runs, etc.). (Bowers) }\end{array}$ & & $\begin{array}{l}\text { (cp)(1) Not accepted. Type of lines identified on } \\
\text { civil drawings and actual line numbers can be found } \\
\text { on piping drawings. } \\
\text { (2)Accepted. Put a clarification note on drawing } \mathrm{H}- \\
\text { 14-100998. }\end{array}$ & \\
\hline 79 & $\begin{array}{l}\text { General comment: Evaluate altering slope of new pipeline } \\
\text { shown on civil piping plans/profiles to minimize need for } \\
\text { shoring during construction. (Boes) }\end{array}$ & & (cp) Accepted. Changed some slopes. & \\
\hline 80 & H-14-100995, sht. 1: no comment. & & N/A & \\
\hline 81 & H-14-100995, sht. 2: no comment. & & N/A & \\
\hline 82 & $\begin{array}{l}\text { H-14-100998, sht. 1: Add symbol for steam pipe supports to } \\
\text { Legend (used on 996, sh. 1). (Boes) }\end{array}$ & & Accepted. Symbol added. & \\
\hline $83^{\circ}$ & $\begin{array}{l}\text { General comment: Where practical, keep pipe slope to no } \\
\text { greater than } 10 \% \text {. (Rifaey) }\end{array}$ & & $\begin{array}{l}\text { (cp) Accepted. Changed slope on various sections } \\
\text { where possible. }\end{array}$ & \\
\hline 84 & H-14-100996, sht. 1 - no comment. & & $\mathrm{N} / \mathrm{A}$ & \\
\hline 85 & H-14-100996, sht. 2 - no comment. & & $\mathrm{N} / \mathrm{A}$ & \\
\hline 86 & $\begin{array}{l}\text { General comment: Check with Operations Compliance on } \\
\text { acceptability with permanently taking out of service existing } \\
\text { waste transfer lines. (Bowers) }\end{array}$ & & $\begin{array}{l}K \text {. Boes checked with } P \text {. Miller and he states it is } \\
\text { acceptable to cut, cap, and abandon the transfer } \\
\text { lines. }\end{array}$ & \\
\hline 87 & $\begin{array}{l}\text { General comment: Recommend developing contingency planning } \\
\text { prior to pipeline construction to deal with unforeseen } \\
\text { underground interferences/conditions (high rad). (Bowers) }\end{array}$ & & (cp) Noted. & \\
\hline $88^{\circ}$ & H-14-100997 - no comments. & & $\mathrm{N} / \mathrm{A}$ & $\underline{\bar{\Sigma}}$ \\
\hline 89 & $\begin{array}{l}\text { H-14-100998: In Note 2. Clarify that reference measurements } \\
\text { are from centerline of 3-inch pipe. (Bowers) }\end{array}$ & & (cp) Accepted. Clarified in Note 2. & 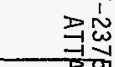 \\
\hline & $0.1(03 / 92)$ WEF011 & & & 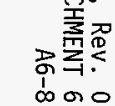 \\
\hline
\end{tabular}




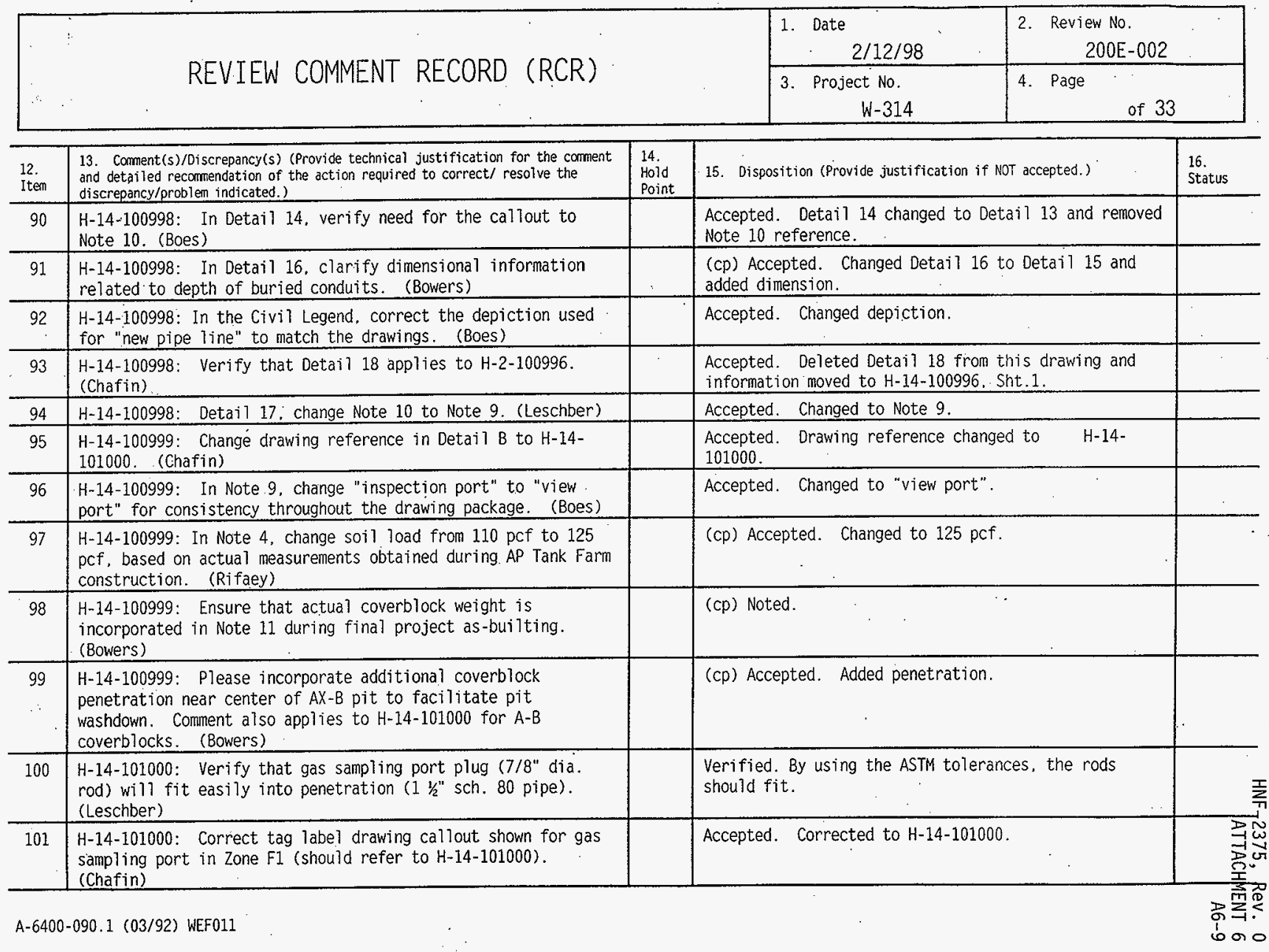




\section{REVIEW COMMENT RECORD (RCR)}

\begin{tabular}{|l|l|}
\hline $\begin{array}{l}\text { 1. Date } \\
2 / 12 / 98\end{array}$ & $\begin{array}{l}\text { 2. Review No. } \\
200 \mathrm{E}-002\end{array}$ \\
\hline $\begin{array}{c}\text { 3. Project No. } \\
W-314\end{array}$ & 4. Page $\quad$ of 33 \\
\hline
\end{tabular}

\begin{tabular}{|c|c|c|c|c|}
\hline $\begin{array}{l}12 . \\
\text { Item }\end{array}$ & $\begin{array}{l}\text { 13. Comment(s)/Discrepancy(s) (Provide technical justification for the corment } \\
\text { and detailed recomendation of the action required to correct/ resolve the } \\
\text { discrepancy/problem indicated.) }\end{array}$ & $\begin{array}{l}14 . \\
\text { Hold } \\
\text { Point } \\
\end{array}$ & 15. Disposition (Provide justification if NOT accepted.) & $\begin{array}{l}16 . \\
\text { Status }\end{array}$ \\
\hline 102 & $\begin{array}{l}\text { H-14-101000: Evaluate repositioning coverblock bails to } \\
\text { ensure safe lifting. (Scott) }\end{array}$ & & (cp) Accepted. See item 103. & \\
\hline 103 & $\begin{array}{l}\text { H-14-101001: Consider use of commercially available lifting } \\
\text { bails in place of specially fabricated bails for the new } \\
\text { coverblocks. if cost effective. (Scott) }\end{array}$ & & $\begin{array}{l}\text { (cp) Accepted. Changed to a commerically available } \\
\text { socket and hook. }\end{array}$ & \\
\hline 104 & H-14-101001: Delete Note 3. (Boes) & & Accepted. Note 3 deleted. & \\
\hline 105 & $\begin{array}{l}\text { H-14-101001: Clarify required pipe grades for carbon steel } \\
\text { and stainless steel, as appropriate, for coverblock } \\
\text { penetration piping. (Leschber) }\end{array}$ & & $\begin{array}{l}\text { (cp) Accepted. Added pipe grades to c-spec, section } \\
05500 \text {. para. } 2.2 .3 \text {. }\end{array}$ & \\
\hline 106 & $\begin{array}{l}\text { H-14-101001: Revise weld symbol shown in Detail } 4 \text { for angle } \\
\text { joint. (Scott) }\end{array}$ & & Accepted. Revised weld symbol. & \\
\hline 107 & H-14-101002, sht. 1 and 2 - no comments. & & N/A & \\
\hline 108 & $\begin{array}{l}\text { General comment: Any existing loose electrical boxes adjacent } \\
\text { to pit wall should be reattached to outside of new shielding } \\
\text { collar. (Bowers) }\end{array}$ & . & (cp) Accepted. - & \\
\hline 109 & $\begin{array}{l}\text { H-14-101002, sht. 3: In Elevation D. change callout for } \\
\text { Detail J to Detail B. (Rickenbach) }\end{array}$ & & Accepted. Changed to "B". & \\
\hline 110 & $\begin{array}{l}\text { H-14-101002. sht. } 3 \text { : In Detail B for the pit shielding } \\
\text { collar, change materials used for railing post sockets to } \\
\text { galvanized to minimize deterioration from rusting (sockets } \\
\text { will collect rain water). (Scott) }\end{array}$ & & (cp) Accepted. Changed to "galvanized" & \\
\hline 111 & H-14-101002, sht. 4 - no comments. & & $N / A$ & \\
\hline 112 & Not used. & & N/A & \\
\hline 113 & $\begin{array}{l}\text { C2. section 15493: Provide appropriate requirements for } \\
\text { controlling storage and pre-heating of weld rod materials to } \\
\text { assure that welds are sound. (Rifaey) }\end{array}$ & & $\begin{array}{l}\text { (cp) Welding filler material control is covered in } \\
\text { the genera] procedures of the FDNW Welding Manual } \\
\text { (FONW-W5-8). }\end{array}$ & $\sum_{\Sigma}^{I}$ \\
\hline \multicolumn{5}{|c|}{ 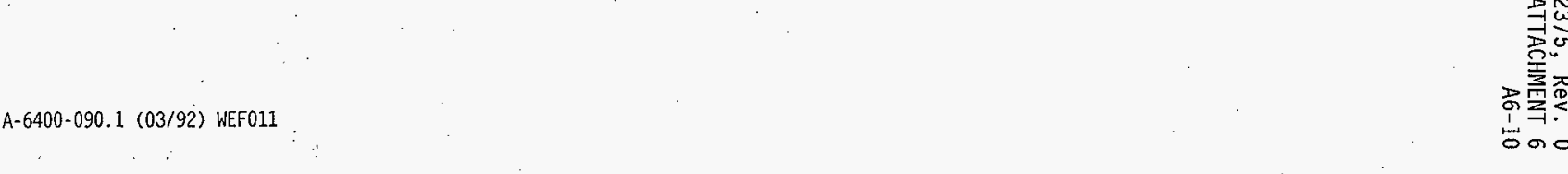 } \\
\hline
\end{tabular}




\begin{tabular}{|c|c|c|}
\hline & $\begin{array}{l}\text { 1. Date } \\
2 / 12 / 98\end{array}$ & $\begin{array}{l}\text { 2. Review No. } \\
200 \mathrm{E}-002\end{array}$ \\
\hline$\therefore \quad$ REVIEW CC & $\begin{array}{r}\text { 3. Project No. } \\
\text { W-314 }\end{array}$ & $\begin{array}{l}\text { 4. Page } \\
\text { of } 33\end{array}$ \\
\hline
\end{tabular}

\begin{tabular}{|c|c|c|c|c|}
\hline $\begin{array}{l}12 . \\
\text { Iten }\end{array}$ & $\begin{array}{l}\text { 13. Comment(s)/Discrepancy(s) (Provide technical justification for the comment } \\
\text { and detailed recommendation of the action required to correct/ resolve the } \\
\text { discrepancy/problem indicated.) }\end{array}$ & $\begin{array}{l}14 . \\
\text { Hold } \\
\text { Point }\end{array}$ & 15. Disposition (Provide justification if NOT accepted.) & $\begin{array}{l}16 . \\
\text { Status }\end{array}$ \\
\hline 114 & $\begin{array}{l}\text { C2. section 15493: If pipe bending less. than 50. provide } \\
\text { measurement of pipe wall thickness. (Rifaey) }\end{array}$ & & $\begin{array}{l}\text { (cp) Noted. The code requires the minimum thickness } \\
\text { for the design pressure be met ( } 0.06 \text { " for primary and } \\
0.08 \text { " for secondary). There are two bends that are } \\
\text { less than } 50 \text { (they are approximately } 4.50) \text {. Since our } \\
\text { starting thickness is much greater than the required } \\
\text { thickness for the pressure (approximately } 0.2^{\prime \prime} \text { ) and } \\
\text { since the bends are not extreme, it is the opinion of } \\
\text { the design engineer that thickness measurements are } \\
\text { not required. }\end{array}$ & \\
\hline 115 & $\begin{array}{l}\text { C2, section 15493, M9 table: For } 3 \text {-inch and smaller piping. } \\
\text { specify butt welding. (Rifaey) }\end{array}$ & & (CD) Accepted. Pipe will be buttwelded. & \\
\hline 116 & $\begin{array}{l}\text { C2, section 15493: Specify weld end details on drawings, and } \\
\text { consider use of pre-prepped fittings to minimize construction } \\
\text { costs. (Rifaey) }\end{array}$ & & $\begin{array}{l}\text { (cp) Accepted. Pipe will be purchased with end } \\
\text { prepped and fittings are butt welded in accordance } \\
\text { with ASME B16.9. . Will add requirement for end prep } \\
\text { to be plain bevel. }\end{array}$ & \\
\hline 117 & $\begin{array}{l}\text { C2, section 15493, para. 3.2.1.3: Specify "normal service". } \\
\text { (Rifaey) }\end{array}$ & & $\begin{array}{l}\text { (cp) Accepted. "Normal service" added to section } \\
3.2 .1 .4 \text { (added new } 3.2 .1 .3 \text { ). }\end{array}$ & \\
\hline 118 & $\begin{array}{l}\text { H-14-101047: Delete temporary jumper between Nozzle R16 and } \\
\text { Nozzle E. (not required due to acceleration of W-314's new } \\
\text { transfer line replacing SL-502). Provide a process blank on } \\
\text { Nozzle E. (Bowers) }\end{array}$ & & (cp) Accepted. Deleted jumper. & \\
\hline 119 & $\mathrm{H}-14-101048$ - no comments. & & $\mathrm{N} / \mathrm{A}$ & \\
\hline 120 & $\begin{array}{l}\text { H-14-101051: Delete jumper "R16-(E)" in the pit jumper } \\
\text { arrangement drawing, as discussed above for the P\&ID. } \\
\text { (Bowers) }\end{array}$ & & (cp) Accepted. See item 118. & . \\
\hline 121 & $\begin{array}{l}\text { H-14-101051: In Note } 6 \text {, clarify that the new encasement pipes } \\
\text { for } 3^{\prime \prime} \text { SN-633 and } 3^{\prime \prime} \text { SN- } 632 \text { are also Safety Class, and that } \\
\text { all other systems, structures, and components depicted on this } \\
\text { drawing are general service. (Leschber) }\end{array}$ & & $\begin{array}{l}\text { (Cp) Accepted. Added SN-633 \& } 632 \text { as SC, left rest } \\
\text { of note same. }\end{array}$ & $\underset{⿱ 亠 䒑}{1}$ \\
\hline \multicolumn{5}{|c|}{ 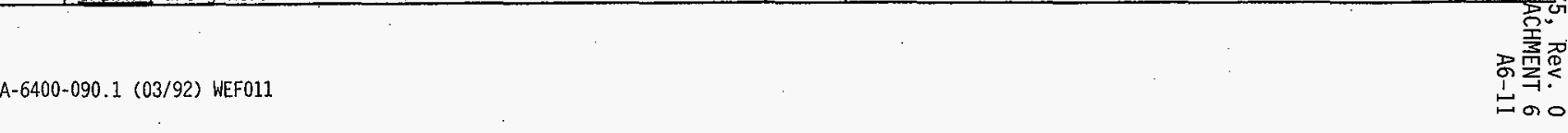 } \\
\hline
\end{tabular}




\begin{tabular}{|c|l|l|}
\hline REVIEW COMMENT RECORD (RCR) & $\begin{array}{l}\text { 1. Date } \\
2 / 12 / 98\end{array}$ & $\begin{array}{r}\text { Review No. } \\
200 \mathrm{E}-002\end{array}$ \\
\cline { 2 - 3 } & $\begin{array}{c}\text { 3. Project No. } \\
\text { W-314 }\end{array}$ & $\begin{array}{l}\text { P. Page } \\
\text { of } 33\end{array}$ \\
\hline
\end{tabular}

\begin{tabular}{|c|c|c|c|c|}
\hline $\begin{array}{l}12 . \\
\text { Item }\end{array}$ & $\begin{array}{l}\text { 13. Comment(s)/oiscrepancy(s) (Provide technical justification for the coment } \\
\text { and detailed recommendation of the action required to correct/ resolve the } \\
\text { discrepancy/problem indicated.) }\end{array}$ & $\begin{array}{l}14 . \\
\text { hoid } \\
\text { Point } \\
\end{array}$ & 15. Disposition (Provide justification if Nor accepted.) & $\begin{array}{l}16 . \\
\text { Status }\end{array}$ \\
\hline 122 & $\begin{array}{l}\text { H-14-101052: Verify whether "existing support structure" } \\
\text { identified on pit floor and to be removed by this project is } \\
\text { actually present. Existing video footage should be checked. } \\
\text { (Bowers) }\end{array}$ & & Verified existing structures still in place. & \\
\hline 123 & $\begin{array}{l}\text { General comment: Consider alternatives (which would } \\
\text { facilitate jumper installation) to the current support } \\
\text { footings provided on } W-314 \text { jumpers. (Pieper) }\end{array}$ & & $\begin{array}{l}\text { (cp) Accepted. Reversed the bolt so that adjustments } \\
\text { can be made from top. This was done based on input } \\
\text { from construction on Project } W-320 \text {. The only concern } \\
\text { was to not have to touch the part of the bolt that } \\
\text { came in contact with the pit. }\end{array}$ & \\
\hline 124 & $\mathrm{H}-14-101061$, shts. 1 and 2 - no comments. & & N/A & \\
\hline 125 & $\mathrm{H}-14-101062$ - no comments. & & N/A & \\
\hline 126 & $\begin{array}{l}\text { H-14-101063: Based on comment above, recommend finalizing } \\
\text { this drawing of the "R16-(E)" jumper for future use, if } \\
\text { needed, but do not include as a part of the W- } 314 \\
\text { construction package. (Bowers) }\end{array}$ & & $\begin{array}{ccc}\text { (cp) Not accepted. } & \text { Drawing removed from package. } \\
\cdot & \ddots & \\
\end{array}$ & \\
\hline 127 & $\begin{array}{l}\text { H-14-101064: Verify that drain valve can be operated using } \\
\text { the T-handle style actuator. (Bowers) }\end{array}$ & & $\begin{array}{l}\text { Verified. Received vendor fax on } 2 / 24 / 98 \text { stating the } \\
\text { torque on a } 2 " \text { MP ball valve with Kynar seats will be } \\
70 \mathrm{ft} \text { - lbs. The T-handle should be adequate. }\end{array}$ & \\
\hline 128 & $\begin{array}{l}\text { H-14-101070: Although actual coverblock weights will be } \\
\text { verified, painted on coverblocks after fabrication, and } \\
\text { included in final as-built drawings, please make the } \\
\text { approximate coverblock weight shown on this painting diagram } \\
\text { consistent with the weight listed on the structural coverblock } \\
\text { drawing (or vice-versa). (Boes) }\end{array}$ & & Accepted. Modified to match structural drawings. & \\
\hline 129 & $\begin{array}{l}\text { General comment: Verify that stop pins on procured valves are } \\
\text { solid and "operator-proof." (Bowers) }\end{array}$ & & $\begin{array}{l}\text { (cP) Verified. Stop pins are solid pins from } 5 / 16^{\prime \prime} \\
\text { to } 1 / 2^{\prime \prime} \text { in diameter. }\end{array}$ & \\
\hline 130 & $\begin{array}{l}\text { H-14-101070: Delete routing for jumper "R16-(E)" from the AX- } \\
\text { B coverblock painting diagram, based on comment above. } \\
\text { (Bowers) }\end{array}$ & & (cp) Accepted. Deleted routing. & \\
\hline
\end{tabular}




\section{REVIEW COMMENT RECORD (RCR)}

\section{Date \\ 2/12/98}

3. Project No.

W-314
2. Review No.

\section{E-002}

4. Page

of 33

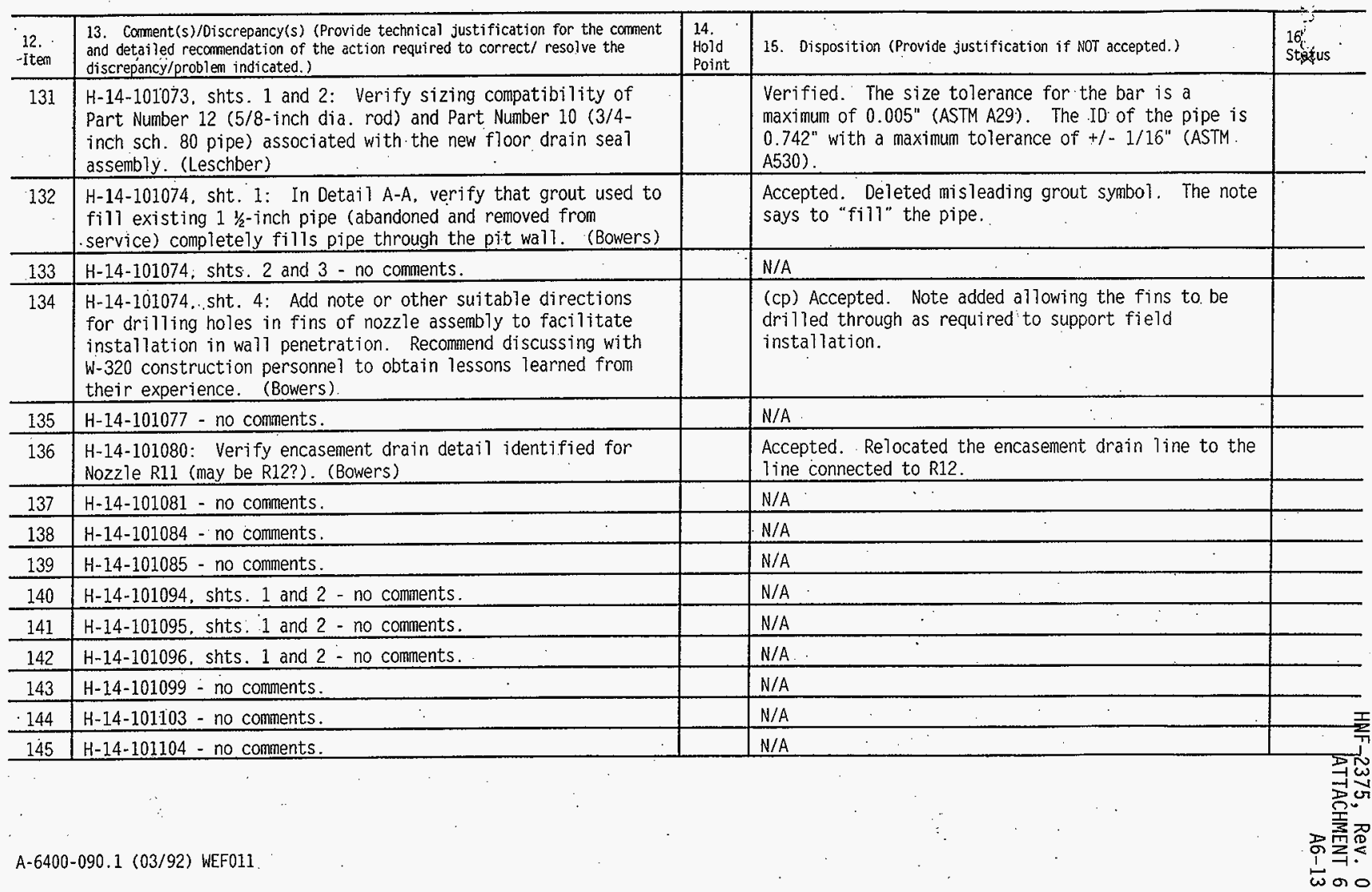


$\begin{array}{r}\text { 1. Date } \\ 2 / 12 / 98 \\ \hline\end{array}$

3. Project No.

W-314
2. Review No. 200E-002

of 33

\begin{tabular}{|c|c|c|c|c|}
\hline 12. & $\begin{array}{l}\text { 13. Comment(s)/Discrepancy(s) (Provide technical justification for the comment } \\
\text { and detailed recommendation of the action required to correct/ resolve the } \\
\text { discrepancy/problem indicated.) }\end{array}$ & $\begin{array}{l}\text { 14. : } \\
\text { Hoid } \\
\text { Point } \\
\end{array}$ & 15. Disposition (Provide justification if NOT accepted.) & $\begin{array}{l}16 . \\
\text { Status }\end{array}$ \\
\hline 146 & $\begin{array}{l}\mathrm{H}-14-101105 \text { : Evaluate method for hydro testing the high point } \\
\text { vent cap, if possible. (Bowers) }\end{array}$ & & $\begin{array}{l}\text { (cp) Accepted. The integrity of the cap will be } \\
\text { verified during the initial hydro test. Re- } \\
\text { verification after draining is not possible. }\end{array}$ & \\
\hline 147 & $\begin{array}{l}\text { General comment: Evaluate potential CDF adhesion to pipeline } \\
\text { and restricting thermal growth. (Bowers) }\end{array}$ & & $\begin{array}{l}\text { (cp) Accepted. Wrapping the pipe with polyethylene } \\
\text { sheeting will be added to structural drawing, H-14- } \\
100998 \text {. }\end{array}$ & \\
\hline 148 & H-14-101110, shts: 1,2 , and 3 - no comments. & & $\mathrm{N} / \mathrm{A}$ & \\
\hline 149 & H-14-101111 - no comments. & & N/A & \\
\hline 150 & $\mathrm{H}-14-101112$ - no comments. & & N/A & \\
\hline 151 & $\mathrm{H}=14-101113$, shts. $1,2,3,4,5$, and 6 - no comments. & & $N / A$ & \\
\hline 152 & $\mathrm{H}-14-101114$ - no comments. & & N/A & \\
\hline 153 & H-14-101116, shts, $1,2,3,4$, and 5 - no comments. & & N/A & \\
\hline 154 & H-14-101117, shts, 1,2 , and 3 - no comments. & & N/A & \\
\hline 155 & H-14-101118, shts. 1 and 2 - no comments. & & $N / A$ & \\
\hline 156 & $\mathrm{H}=14-101118$. sht. 3: Identify partial plan area. (Boes) & & Accepted. Added callout to refer to Detajl 1. & \\
\hline 157 & H-14-101119, shts. 1 and 2 - no comments. & & N/A & \\
\hline 158 & $\begin{array}{l}\text { H-14-101121, shts. } 1 \text { and } 2 \text { : Recommend adding reference tank } \\
\text { elevations to support hydraulic calculations. Also recommend } \\
\text { not issuing these drawings until after completion of remaining } \\
\text { pump pit tie-ins. (Bowers) }\end{array}$ & & $\begin{array}{l}\text { (cP) Accepted. Will add reference information during } \\
\text { later design package }(\mathrm{AZ}) \text {. }\end{array}$ & \\
\hline 159 & $\begin{array}{l}\text { C2, page v: Add hydraulic calculation for line loss to the } \\
\text { listing of calculations. (Leschber) }\end{array}$ & & (cp) Accepted. Calculation number W314-P-101 added. & \\
\hline 160 & $\begin{array}{l}\text { C2, section } 01010-1 \text {, section } 1.2 .1 .1 \text { : Clarify that the major } \\
\text { work to be completed is applicable to pits } A X-B \text { and } A-B \text {, } \\
\text { (Warnick) }\end{array}$ & & Accepted. Clarified scope description. & $\frac{1}{T}$ \\
\hline 161 & $\begin{array}{l}\text { C2. General comment: Specify the date(s) for codes of record } \\
\text { used to support the design. (Leschber) }\end{array}$ & & $\begin{array}{l}\text { (cp) Accepted. Add reference to October } 1997 \text { on all } \\
\text { applicable sections. }\end{array}$ & 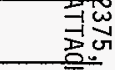 \\
\hline
\end{tabular}




\begin{tabular}{|c|l|l|l|}
\hline \multirow{2}{*}{ REVIEW COMMENT RECORD (RCR) } & $\begin{array}{l}\text { 1. Date } \\
2 / 12 / 98\end{array}$ & $\begin{array}{c}\text { Review No. } \\
200 \mathrm{E}-002\end{array}$ \\
\cline { 2 - 4 } & $\begin{array}{l}\text { 3. Project No. } \\
\text { W-314 }\end{array}$ & $\begin{array}{l}\text { 4. Page } \\
\text { of } 33\end{array}$ \\
\hline
\end{tabular}

\begin{tabular}{|c|c|c|c|c|}
\hline $\begin{array}{l}12 . \\
\text { Item }\end{array}$ & $\begin{array}{l}\text { 13. Comment(s)/Discrepancy(s) (Provide technical justification for the comment } \\
\text { and detailed recormendation of the action required to correct/ resolve the - } \\
\text { discrepancy/problem indicated.) }\end{array}$ & $\begin{array}{l}14 . \\
\text { Hoid } \\
\text { Point }\end{array}$ & 15. Disposition (Provide justification if NOT accepted.) & $\begin{array}{l}16 . \\
\text { Status }\end{array}$ \\
\hline 162 & $\begin{array}{l}\text { C2, } 02237-2 \text {, section 1.2.1: Verify that Section } 2.10 \text { of the } \\
\text { TWRS PPM is a valid reference. (Warnick) }\end{array}$ & & $\begin{array}{l}\text { Verified. This is appropriate until a project } \\
\text { specific procedure for submittal processing is } \\
\text { approved. }\end{array}$ & \\
\hline 163 & $\begin{array}{l}\text { C2. General comment: Ensure that procurement documentation } \\
\text { requires MSOS's for all procured jtems. (Bowers) }\end{array}$ & & Noted. Part of FDNW's standard procurement process. & \\
\hline 164 & $\begin{array}{l}\text { C2, } 03300-3 \text {, section } 1.5 .1 \text { : Evaluate rewording requirement } \\
\text { concerning qualified suppliers meeting NQA-1 program } \\
\text { requirements. Clarify that suppliers don't need to have an } \\
\text { NQA-1 program. (Leschber) }\end{array}$ & & $\begin{array}{l}\text { Not accepted. Wording does not state supplier needs } \\
\text { to have a NQA-1 program but a program that meets the } \\
\text { applicable requirements of NQA-1. }\end{array}$ & \\
\hline 165 & $\begin{array}{l}\text { C2, } 03300-4 \text {. section 1.5.2.2.a: Clarify that testing agency } \\
\text { activities are limited to non-field activities. (Warnick) }\end{array}$ & & Not accepted. Acceptable as worded. & \\
\hline 166 & $\begin{array}{l}\text { C2. 05055-2, section 3.3.1: How will construction personnel } \\
\text { account for rebar encountered during drilling/installation of } \\
\text { anchors? (Leschber) }\end{array}$ & & $\begin{array}{l}\text { FDNW Practice } 134.500 .8209 \text {, as noted in section. } \\
3.3 .1 \text {, spells out what they will do. }\end{array}$ & \\
\hline 167 & $\begin{array}{l}\text { C2, } 05500-3 \text {, section 1.3.2.2.b: Verify whether ASTM A568 is } \\
\text { correct, or should it be A569. (Leschber) }\end{array}$ & & Verified A568 correct. & \\
\hline 168 & $\begin{array}{l}\text { C2. } 05500-4 \text {, section 2.2.3: Add applicable requirements for } \\
\text { stainless steel pipe. (Leschber) }\end{array}$ & & $\begin{array}{l}\text { (CD) Accepted. SST requirement added (ASTM A312. } \\
\text { Type 304C). }\end{array}$ & \\
\hline 169 & $\begin{array}{l}\text { C2. } 05500-5 \text {, section } 2.3 .2: \text { Add a reference in this paragraph } \\
\text { to the coating section of this specification. (Leschber) }\end{array}$ & . & $\begin{array}{l}\text { (cp) Accepted. Reference to coating section } \\
\text { included. }\end{array}$ & \\
\hline 170 & $\begin{array}{l}\text { C2. General comment: Review welding procedures to determine } \\
\text { how they address weld strikes. (Rifaey) }\end{array}$ & & $\begin{array}{l}\text { (cp) Accepted. Added a new requirement (para. } \\
3.1 .3 .7 \text { ) requiring arc strikes to be examined by } \\
\text { liquid. penetrant exam. }\end{array}$ & \\
\hline 171 & $\begin{array}{l}\text { C2, Section } 05500 \text {, General comment: Required tolerances do } \\
\text { not appear. to be identified in the } \mathrm{C} 2 \mathrm{spec} \text { or the drawings for } \\
\text { meta? fabrications. Revise as needed. (Leschber) }\end{array}$ & & $\begin{array}{l}\text { (cp) Accepted. Tolerances }(+/-1 / 8 \text { in.) called out } \\
\text { on drawing H-14-100999. General note.11. }\end{array}$ & \\
\hline 172 & $\begin{array}{l}\text { C2, } 09855-5 \text {, section } 2.2 .3 .8 \text { : Evaluate clarification that } 12- \\
\text { yr design life for coating system is applicable to interior of } \\
\text { pit. or consider revising requirement. (Leschber) }\end{array}$ & & $\begin{array}{l}\text { (CP) Accepted. Added additional requirement for SPC } \\
\text { exposure to solar uv radiation. }\end{array}$ & 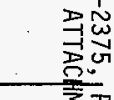 \\
\hline
\end{tabular}




\begin{tabular}{|c|l|l|}
\hline \multirow{2}{*}{ REVIEW COMMENT RECORD (RCR) } & $\begin{array}{l}\text { 1. Date } \\
2 / 12 / 98\end{array}$ & $\begin{array}{r}\text { 2. Review No. } \\
200 \mathrm{e}-002\end{array}$ \\
\cline { 2 - 4 } & $\begin{array}{c}\text { 3. Project No. } \\
\text { W-314 }\end{array}$ & $\begin{array}{c}4 . \quad \text { Page } \\
\text { of } 33\end{array}$ \\
\hline
\end{tabular}

\begin{tabular}{|c|c|c|c|c|}
\hline $\begin{array}{l}\text { 12: } \\
\text { Item }\end{array}$ & $\begin{array}{l}\text { 13. Comment(s)/Discrepancy(s) (Provide technical justification for the corment } \\
\text { and detailed recomendation of the action required to correct/ resolve the } \\
\text { discrepancy/problem indicated.) }\end{array}$ & $\begin{array}{l}\text { 14. } \\
\text { Hoid } \\
\text { Point }\end{array}$ & 15. Disposition (Provide justification if NOT accepted.) & $\begin{array}{l}16 . \\
\text { Status }\end{array}$ \\
\hline 173 & $\begin{array}{l}\text { C2. 09855. General comment: Evaluate need to add requirement } \\
\text { for spark testing coated surfaces of pit for holidays. } \\
\text { (Rifaey) }\end{array}$ & & $\begin{array}{l}\text { (cp) Evaluated. With at least } 3 \text { coats and total } \\
\text { thickness of } 15 \text { mils; holidays should not be thru the } \\
\text { multiple coats to the substrate. Additionally, spark } \\
\text { tests to a concrete surface with embedded steel may } \\
\text { not be accurate and high voltage required may damage } \\
\text { embedded steel. }\end{array}$ & \\
\hline 174 & $\begin{array}{l}\text { C2. 09855-8, section 3.2.2.4: Consider alternatives to using } \\
\text { muriatic acid for cleaning concrete due to possible damage to } \\
\text { metallic items. If used, specify precautions to protect } \\
\text { metallic items. (Bowers) }\end{array}$ & & $\begin{array}{l}\text { (cp) Accepted. Added note to mask exposed metal for } \\
\text { protection from muriatic acid solutions. }\end{array}$ & \\
\hline 175 & $\begin{array}{l}\text { C2. } 09855-10 \text {. section 3.3.6: Correct drawing references } \\
\text { (should be } \mathrm{H}-14-101070 \text { and } \mathrm{H}-14-101103 \text { ). (Leschber) }\end{array}$ & & Accepted. Corrected drawing numbers. & \\
\hline 176 & C2, 09855-11, section 3.4.2: Specify tests required. (Singh) & & $\begin{array}{l}\text { (cp) Accepted. Specified } Q C \text { inspection requirements } \\
\text { for SPC. Deleted reference to any specific tests. }\end{array}$ & \\
\hline 177 & $\begin{array}{l}\text { C2. } 13440-4 \text {, section } 2.2 .2 \text { : Verify specified temperature } \\
\text { range limits. Appears to be a typo error. (Eischens) }\end{array}$ & & Accepted. Changed to " $-50 "$. & \\
\hline 178 & $\begin{array}{l}\text { C2, } 13440-4 \text {, section 2.2.5: Change NEMA } 4 \text { to NEMA } 4 X \text {, as } \\
\text { appropriate. (Eischens) }\end{array}$ & & Accepted. Made a 11 references to NEMA $4 X$. & \\
\hline 179 & $\begin{array}{l}\text { C2, } 13440-4 \text {, section 2.3.2: Consider rewording to allow } \\
\text { latitude in qualifying CGI equipment. (Ri faey) }\end{array}$ & & $\begin{array}{l}\text { (cp) Accepted. Reworded to allow testing by } \\
\text { qualified personnel. }\end{array}$ & \\
\hline 180 & $\begin{array}{l}\text { C2. } 13440-4 \text {, section } 2.3 .1 \text { : Recommend deleting last sentence } \\
\text { of this section dealing with reference to Table } 1 \text { in data } \\
\text { sheets. (Eischens) }\end{array}$ & & $\begin{array}{l}\text { (cp) Accepted. Deleted last part of last sentence at } \\
\text { "... or on..". }\end{array}$ & \\
\hline 181 & $\begin{array}{l}\text { C2. General comment: Review specification and the drawings to } \\
\text { assure consistency in SC equipment/component callouts. } \\
\text { (Eischens) }\end{array}$ & & (cp) Accepted. Verified consistency of SC callouts & \\
\hline 182 & $\begin{array}{l}\text { C2, 13440-4, section 2.3.2.1.b: Revise statement to say that } \\
\text { input voltage sha11 be verified as } 120 \mathrm{~V} \text {. Also delete "1.2V" } \\
\text { and "0.1A." (Eischens) }\end{array}$ & & $\begin{array}{l}\text { Accepted. Completely revised section with new test } \\
\text { procedures in accordance with Design Authority } \\
\text { (Bowers/Scaief) direction. }\end{array}$ & \\
\hline
\end{tabular}




\begin{tabular}{|c|l|l|}
\hline \multirow{2}{*}{ REVIEW COMMENT RECORD (RCR) } & $\begin{array}{l}\text { 1. Date } \\
2 / 12 / 98\end{array}$ & $\begin{array}{c}\text { 2. Review No. } \\
200 \mathrm{E}-002\end{array}$ \\
\cline { 2 - 4 } & $\begin{array}{c}\text { 3. Project No. } \\
\text { W-314 }\end{array}$ & $\begin{array}{l}\text { 4. Page } \\
\text { of } 33\end{array}$ \\
\hline
\end{tabular}

\begin{tabular}{|c|c|c|c|c|}
\hline $\begin{array}{l}12 . \\
\text { Item }\end{array}$ & $\begin{array}{l}\text { 13. Comment(s)/Discrepancy(s) (Provide technical justification for the comment } \\
\text { and detailed recormendation of the action required to correct/ resolve the } \\
\text { discrepancy/problem indicated.) }\end{array}$ & $\begin{array}{l}14 . \\
\text { Hoid } \\
\text { Point } \\
\end{array}$ & 15. Disposition (Provide justification if NOT accepted.) & $\begin{array}{l}16 . \\
\text { Status }\end{array}$ \\
\hline 183 & $\begin{array}{l}\text { C2, 13440-4. section } 2.3 .2 .1 . \mathrm{b} \text { : In first bullet, ensure } \\
\text { associated data sheet calls for } 2 \text { form C contacts. (Singh) }\end{array}$ & & $\begin{array}{l}\text { Accepted. Completely revised section with test } \\
\text { procedures. }\end{array}$ & \\
\hline 184 & $\begin{array}{l}\text { C2. } 13440-4 \text {, section } 2.3 .2 .1 . a: \text { Check negative percentage } \\
\text { range on } 10 \mathrm{~K} \text { resistor. (Singh) }\end{array}$ & & $\begin{array}{l}\text { (cp) Accepted. Completed revised section with test } \\
\text { procedures. }\end{array}$ & \\
\hline 185 & $\begin{array}{l}\text { C2, } 13440-5 \text {, section } 2.3 .2 .1 \text { b: Verify need for relay to } \\
\text { function in an envïronment of } 0-100 \% \text { relative humidity. }\end{array}$ & & $\begin{array}{l}\text { Accepted. Deleted humidity requirement as a critical } \\
\text { charateristic. Completely revised section with test } \\
\text { procedures. }\end{array}$ & \\
\hline 186 & $\begin{array}{l}\text { C2. Evaluate need for adding a temperature alarm in the relay } \\
\text { cabinet. (Singh) [If determined to need temperature alarm, } \\
\text { notify J.R. LaPointe - TWRS Maintenance] }\end{array}$ & & $\begin{array}{l}\text { (cp) Not accepted. Temperature alarm is not needed } \\
\text { or desired. }\end{array}$ & \\
\hline 187 & $\begin{array}{l}\text { C2, } 13440-11 \text { : Add missing label ("Dimensional Drawings") to } \\
\text { first column in vendor information list. (Bowers) }\end{array}$ & & Accepted. Corrected. & \\
\hline 188 & $\begin{array}{l}\text { C2, 13440-13: Delete encasement leak detection relay from } \\
\text { Table } 1 \text { (it does not need to be qualified). (Eischens) } \\
\end{array}$ & & $\begin{array}{l}\text { (cp) Not accepted. Changed tables to distinguish SC. } \\
\text { items. }\end{array}$ & . \\
\hline 189 & $\begin{array}{l}\text { C2, } 13440 \text {, General comment: Ensure that vendor information is } \\
\text { provided for all procured items. (Bowers) }\end{array}$ & & $(c p)$ Noted. & \\
\hline 190 & $\begin{array}{l}\text { C2, 13440-12: In item } 7 \text { of the data sheet, add " } 60 \text { Hertz" for } \\
\text { consistency with other data sheets (this is also a general } \\
\text { comment for all data sheets, as applicable). Also, in item } \\
12 \text {, specify } 120 \mathrm{~V} \text { ac rating. as applicable. (Singh) } \\
\end{array}$ & & $\begin{array}{l}\text { Accepted. Added "60 Hertz". Also added } 250 \mathrm{~V} \text { ac } \\
\text { rating. }\end{array}$ & \\
\hline 191 & $\begin{array}{l}\text { C2, } 13440-14: \text { In item } 5 \text { of the data sheet, verify correct } \\
\text { model number (Singh) }\end{array}$ & & Accepted. Corrected model number. & \\
\hline 192 & $\begin{array}{l}\text { C2. 13440-14: In item } 16 \text { of the data sheet, add mode] number. } \\
\text { (Eischens) }\end{array}$ & & Accepted. Added model number. & \\
\hline 193 & $\begin{array}{l}\text { C2, 13440, General comment: For a11 data sheets, check } \\
\text { contact type. (Singh) }\end{array}$ & & $\begin{array}{l}\text { Accepted. Verified and corrected as required all } \\
\text { contact types. }\end{array}$ & $\sum_{\text {而 }}$ \\
\hline 194 & $\begin{array}{l}\text { C2. } 13440-17: \text { Add table to the data sheet for leak detection } \\
\text { probes. (Eischens) }\end{array}$ & & (cp) Accepted. Added table. & 국 \\
\hline
\end{tabular}




\begin{tabular}{|c|l|l|l|}
\hline REVIEW COMMENT RECORD (RCR) & $\begin{array}{l}\text { Date } \\
2 / 12 / 98\end{array}$ & $\begin{array}{c}\text { Review No. } \\
200 \mathrm{E}-002\end{array}$ \\
\cline { 2 - 4 } & $3 . \quad \begin{array}{c}\text { Project No. } \\
\text { W-314 }\end{array}$ & $\begin{array}{l}\text { 4. Page } \\
\text { of } 33\end{array}$ \\
\hline
\end{tabular}

\begin{tabular}{|c|c|c|c|c|}
\hline $\begin{array}{l}12 . \\
\text { Item }\end{array}$ & $\begin{array}{l}\text { 13. Comment(s)/Discrepancy(s) (Provide technical justification for the comment } \\
\text { and detailed recommendation of the action required to correct/ resolve the } \\
\text { discrepancy/problen indicated.) }\end{array}$ & $\begin{array}{l}14 . \\
\text { Hold } \\
\text { Point }\end{array}$ & 15. Disposition (Provide justification if NOT accepted.) & $\begin{array}{l}16 . \\
\text { Status }\end{array}$ \\
\hline 195 & $\begin{array}{l}\text { C2, } 13440 \text {. General comment: Verify field temperature for } \\
\text { relay cabinets. (Singh) }\end{array}$ & & (cp) Accepted. Revised temperature to 140 degrees F. & \\
\hline 196 & $\begin{array}{l}\text { C2, 13440-18: Add appropriate requirements for thermostatic } \\
\text { control. (Singh) }\end{array}$ & & Accepted. Added requirement to sheet. & \\
\hline 197 & C2, 22 Add appropriate VA rating to this data sheet. (Singh) & & (cp) Accepted. Added VA requirement. & \\
\hline 198 & $\begin{array}{l}\text { General comment: Specify on drawings where to land the leads } \\
\text { for a specified voltage. (Singh) }\end{array}$ & & (cp) Accepted. Added to drawings. & \\
\hline 199 & $\begin{array}{l}\text { C2, 15493-5, 2.2.3.1: Verify use of teflon paste - consistent } \\
\text { with AN's C1 spec? (Leschber) }\end{array}$ & & $\begin{array}{l}\text { Verified teflon paste acceptable, but added } \\
\text { requi rement for use of pipe joint compound } L \dot{A}-C D-T-0- \\
T \text { in flammable gas areas. }\end{array}$ & \\
\hline 200 & $\begin{array}{l}\text { C2, } 15493-8,3.1 .3 .5: \text { Verify that TIG is used for first pass } \\
\text { on pipe welds. as appropriate. (Bowers) }\end{array}$ & & $\begin{array}{l}\text { (cp) Accepted. Added requirement to TIG all welds } \\
\text { where weld splatter is a concern. }\end{array}$ & \\
\hline 201 & $\begin{array}{l}\text { C2, }-10: 3.2 .2 \text { and } 3.2 .3: \text { Evaluate whether water quality } \\
\text { needs to. be specified for flushing and cleaning of piping. } \\
\text { (Leschber) }\end{array}$ & & $\begin{array}{l}\text { (cp) Evaluated. No changes reuired. Raw water used } \\
\text { for flushing al ready has a chloride. content of less } \\
\text { than } 10 \mathrm{ppm} \text {. }\end{array}$ & \\
\hline 202 & $\begin{array}{l}\text { C2, } 15493-10 \text {, section 3.2.3.2: Specify cleanliness standard } \\
\text { required for constructed piping. (LaPointe) }\end{array}$ & & $\begin{array}{l}\text { (cp) Accepted. Added requirement that no "visible" } \\
\text { dirt. scale, or debris is present. }\end{array}$ & \\
\hline 203 & $\begin{array}{l}\text { C2, 15493, General comment: Ensure that appropriate } \\
\text { requirements are specified to control chloride/fluoride } \\
\text { exposure to stainless steel piping. (Bowers) }\end{array}$ & & $\begin{array}{l}\text { (cp) Accepted. Added requirement for marking pens in } \\
\text { paragraph 3.1.4.3. Tape already covered in paragraph } \\
2.2 .4 .1 \text {. Flush water addressed in item } 201 \text { and } \\
\text { tooling covered in paragraph } 3.1 .1 .5 \text {. }\end{array}$ & \\
\hline 204 & $\begin{array}{l}\text { C2. } 15493-13 \text {, section } 3.3 .1 \text { : Evaluate suitability/benefit of } \\
\text { additional inspection requirements (beyond B31.3 code } \\
\text { requirements) for SC piping. (Bowers) }\end{array}$ & & $\begin{array}{l}\text { (cp) Not accepted. Code requirements provide } \\
\text { necessary requirements for safe design and } \\
\text { construction of pressure piping. }\end{array}$ & \\
\hline 205 & $\begin{array}{l}\text { C2. 15493-16: Add " } x \text { " in column for circuit diagrams for } \\
\text { position indicators. (Bowers) }\end{array}$ & & (cp) Accepted. Added " $x$ "'s. & 폴 \\
\hline 206 & $\begin{array}{l}\text { C2. 15493-A-4, section 3.1.1: Verify correct spelling of } \\
\text { "Amerlock". (Leschber) }\end{array}$ & & Spelling verified to be correct as shown. & \\
\hline \multicolumn{3}{|c|}{ A-6400-090.1 (03/92) WEF011 } & . & 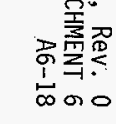 \\
\hline
\end{tabular}




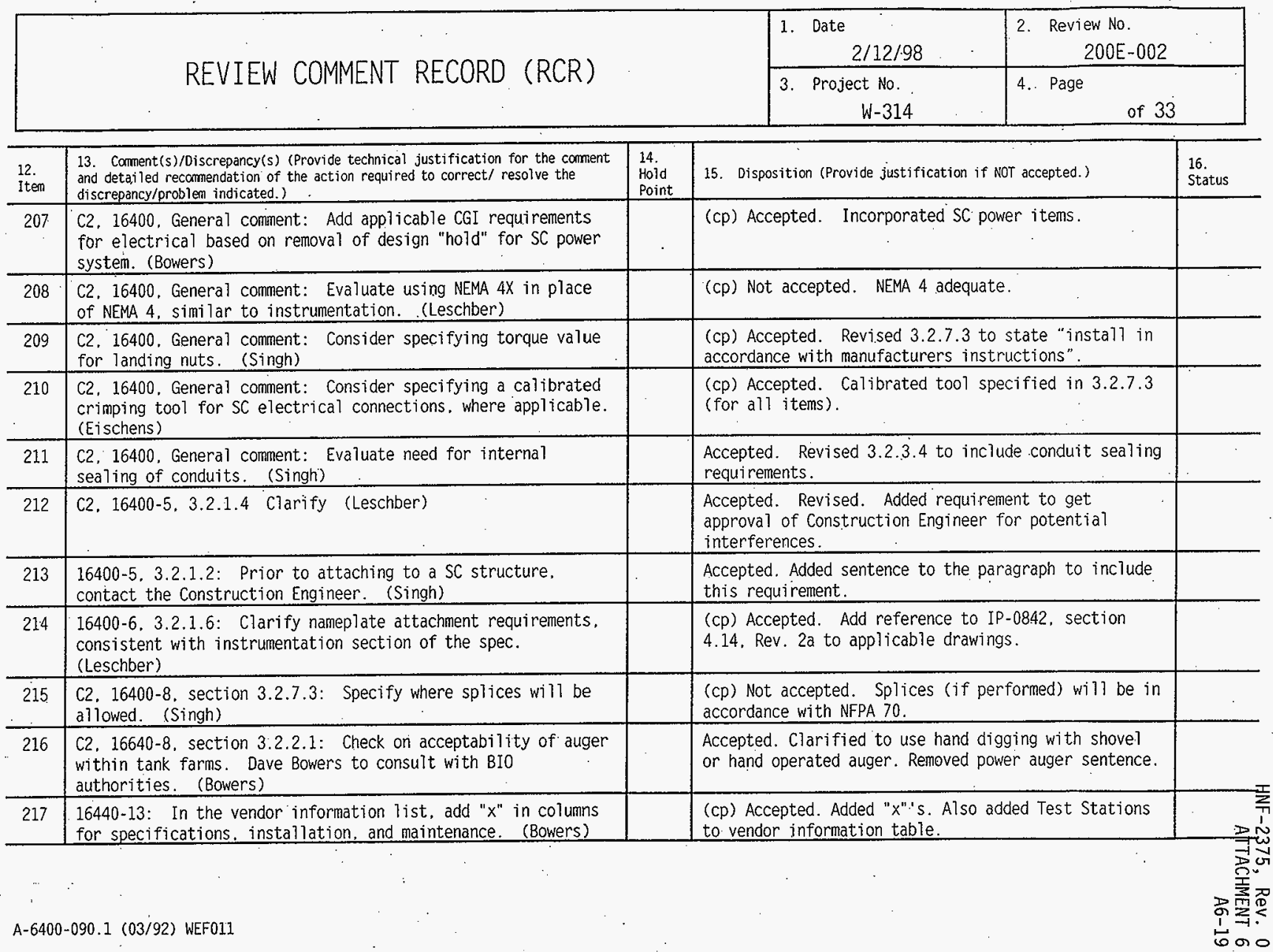




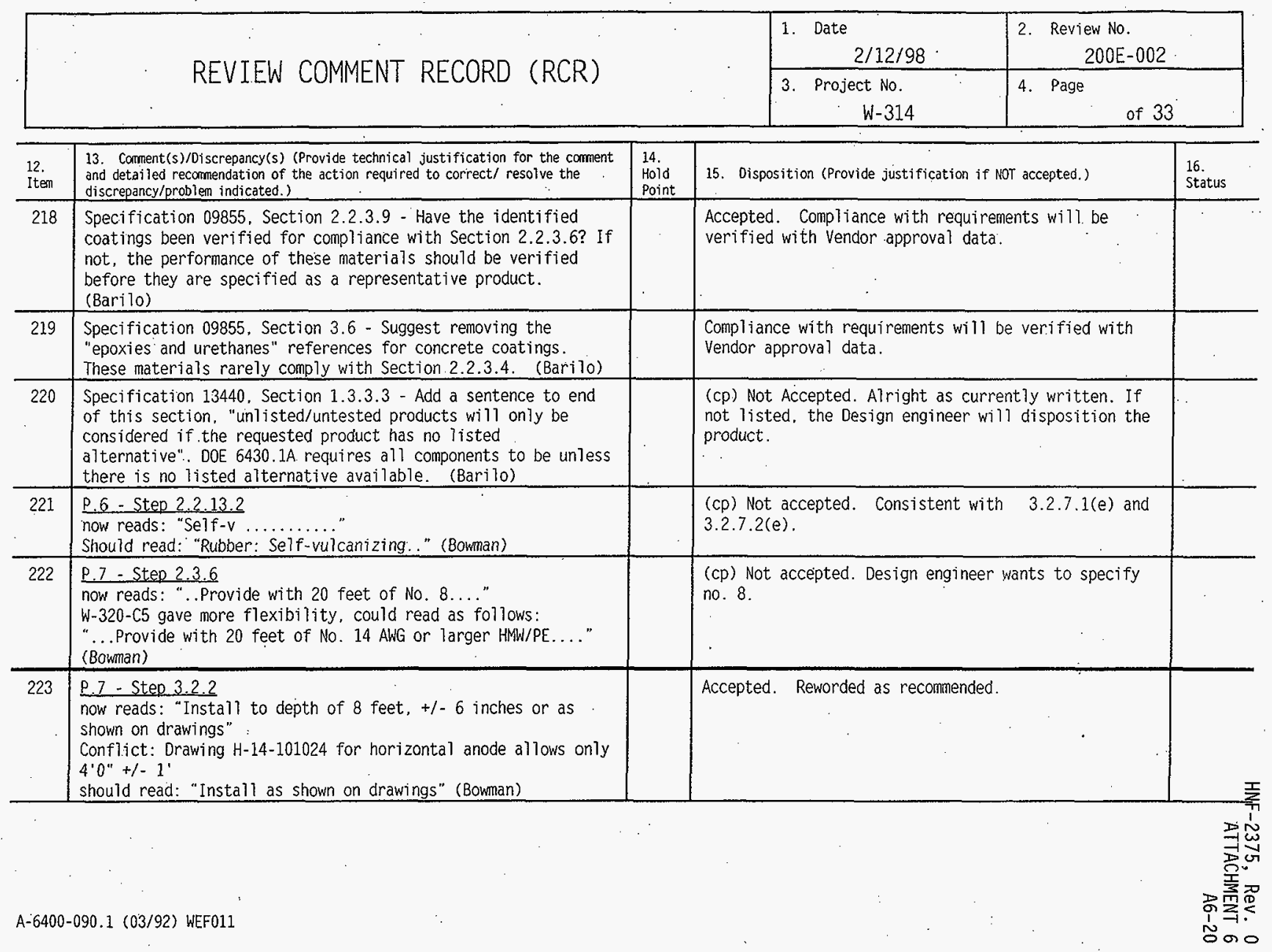




\begin{tabular}{|l|l|l|}
\hline \multirow{2}{*}{ REVIEW COMMENT RECORD (RCR) } & $\begin{array}{l}\text { 1. Date } \\
2 / 12 / 98\end{array}$ & $\begin{array}{c}\text { Review No. } \\
200 \mathrm{E}-002\end{array}$ \\
\cline { 2 - 4 } & $\begin{array}{l}\text { 3. Project No. } \\
\text { W-314 }\end{array}$ & $\begin{array}{l}\text { 4. Page } \\
\text { of } 33\end{array}$ \\
\hline
\end{tabular}

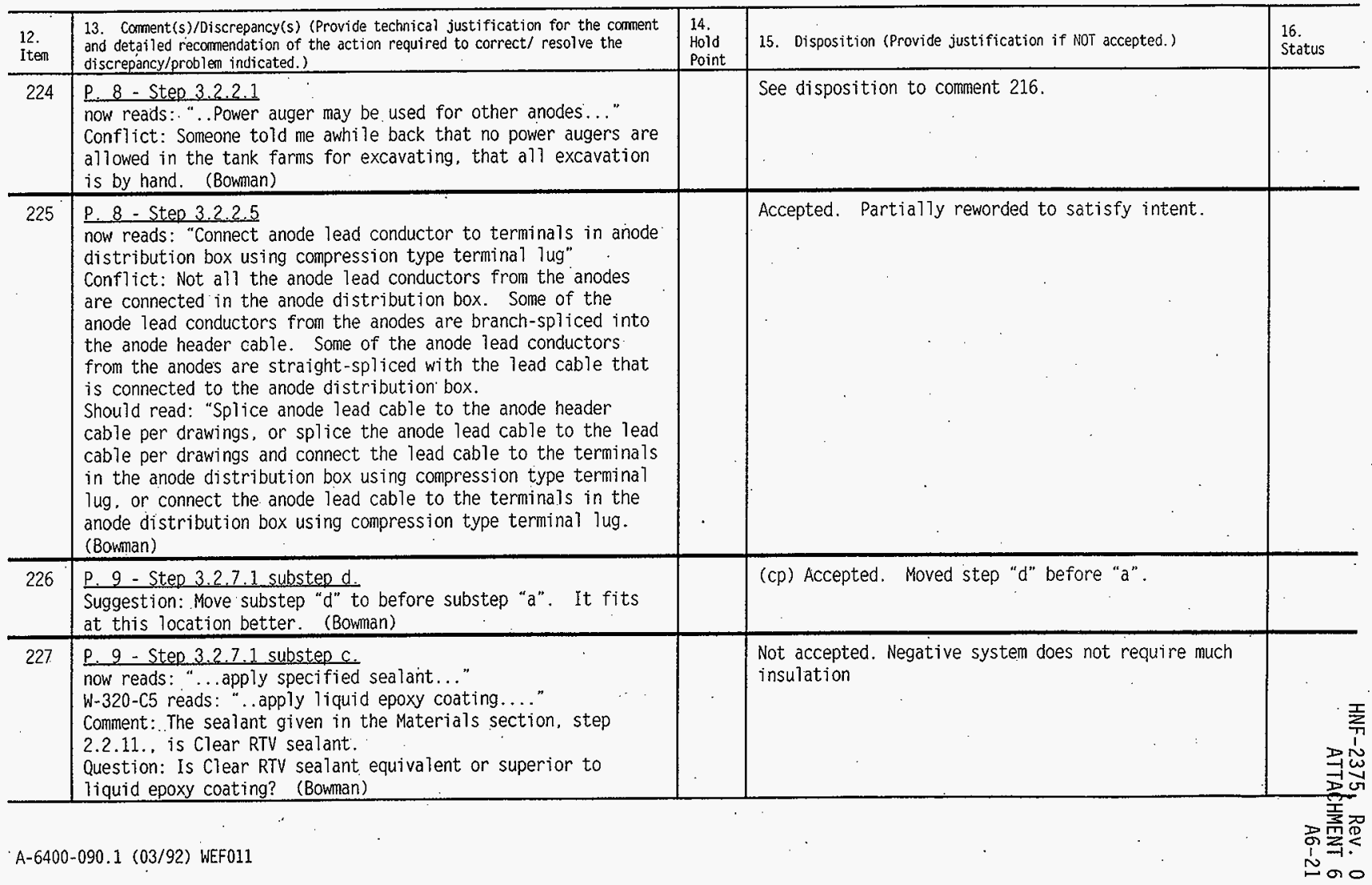




\section{REVIEW COMMENT RECORD (RCR)}

\begin{tabular}{|l|l|}
\hline $\begin{array}{l}\text { 1. Date } \\
2 / 12 / 98\end{array}$ & $\begin{array}{l}\text { 2. Review No. } \\
200 E-002\end{array}$ \\
\hline $\begin{array}{c}\text { 3. Project No. } \\
\text { W-314 }\end{array}$ & 4. Page: \\
\hline
\end{tabular}

\begin{tabular}{|c|c|c|c|c|}
\hline $\begin{array}{l}12 . \\
\text { Item }\end{array}$ & $\begin{array}{l}\text { 13. Corment(s)/oiscrepancy(s) (Provide technical justification for the comment } \\
\text { and detailed recommendation of the action required to correct/ resolve the } \\
\text { discrepancy/problem indicated.) }\end{array}$ & \begin{tabular}{|l|}
14. \\
Hold \\
Point
\end{tabular} & 15. Disposition (Provide justification if NOT accepted.) & $\begin{array}{l}16 . \\
\text { Status }\end{array}$ \\
\hline 228 & $\begin{array}{l}\text { P. } 9 \text { - Step } 3.2 .7 .1 \text { substep } f \text {. substep } 3 \\
\text { Question: Is the plastic sheet material of this substep } \\
\text { "Bubblewrap"? (Bowman) }\end{array}$ & & $\begin{array}{l}\text { Unknown. The section was clarified to repair to } \\
\text { whatever the original material is. }\end{array}$ & \\
\hline 229 & $\begin{array}{l}\text { P.10 - Step } 3.2 .7 .2 \text { substep } d \\
\text { Suggestion: Move substep " } d \text { " to before substep "a". It fits } \\
\text { at this location better. (Bowman) }\end{array}$ & - & (cp) Accepted. Moved step " $\mathrm{d}$ " before "a". & \\
\hline 230 & $\begin{array}{l}\text { P. } 10 \text { - Step } 3.2 .7 .2 \text { substep } c \\
\text { now reads: "Apply clear RTV si7icon sealant..." } \\
\text { W-320-C5 reads: ".. apply liquid epoxy coating..." } \\
\text { Question: Is Clear RTV sealant equivalent or superior to } \\
\text { liquid epoxy coating? (Bowman) }\end{array}$ & & See disposition to comment 227. & \\
\hline 231 & $\begin{array}{l}\frac{\text { p. } 10-\text { Step } 3.2 .8}{\text { now reads: "Instal1 } 1 \text { anode feeder, anode loop, anode lead, and }} \\
\text { negative return cables as follows." } \\
\text { should read: "Install anode header, anode loop, lead, anode } \\
\text { lead, and negative return cables as follows." (Bowman) }\end{array}$ & & Accepted. Reworded as recommended. & \\
\hline
\end{tabular}




\begin{tabular}{|c|c|c|c|c|c|c|}
\hline & \multirow{2}{*}{ REVIEW COMMENT RECORD（RCR） } & & & $\begin{array}{l}\text { 1. Date } \\
2 / 12 / 98 \\
\end{array}$ & \multicolumn{2}{|c|}{$\begin{array}{l}\text { 2. Review No. } \\
200 \mathrm{E}-002\end{array}$} \\
\hline & & & & $\begin{array}{r}\text { 3. Project No. } \\
\text { W-314 }\end{array}$ & 4. Page & \\
\hline $\begin{array}{l}12 . \\
\text { Item } \\
\end{array}$ & $\begin{array}{l}\text { 13. Comment(s)/Discrepancy(s) (Provide technical justification for the comment } \\
\text { and detailed recomendation of the action required to correct/ resolve the } \\
\text { discrepancy/problem indicated.) }\end{array}$ & $\begin{array}{l}14 . \\
\text { Hold } \\
\text { Point }\end{array}$ & 15. Dispos & ition (Provide justifica & accepted.) & $\begin{array}{l}16: \\
\text { Status } \\
\end{array}$ \\
\hline 232 & $\begin{array}{l}\text { P. } 10 \text { - Step } 3.2 .8 .3 \\
\text { now reads: "Terminate anode feeder and anode loop cable at } \\
\text { junction box or splice points..." } \\
\text { Comment: feeder should be header, a rectifier and distribution } \\
\text { boxes are on the drawings and also receive these cables, and } \\
\text { the header and loop cable terminations are only spliced in } \\
\text { junction boxes. } \\
\text { should read: "Terminate anode header and anode loop cables at } \\
\text { junction boxes. or distribution boxes, or at the rectifier per } \\
\text { drawings..." } \\
\text { now reads: "...Terminate negative return cable at splice point } \\
\text { and on pipe where shown on Drawings.". } \\
\text { Comment: the only negative return cable of W-314 on drawing } \\
\text { H-14-101020 goes from rectifier } 12 \text { to pipe SN-630 } \\
\text { should read: ".. Terminate negative return cable at rectifier } \\
\text { and on pipe where shown on Drawings." } \\
\text { now reads: "... Use terminal lugs for conductor terminations } \\
\text { at anode junction boxes..." } \\
\text { Comment: Anode junction boxes, at step } 3.2 .5 \text { of this } \\
\text { construction specification, says "Join conductors. with } \\
\text { compression connectors or split bolts, and insulate with } \\
\text { electrical tape or insulating boot". The newly installed } \\
\text { anode junction boxes are empty and have no existing terminal } \\
\text { jugs. There are }\end{array}$ & & $\begin{array}{l}\text { Accepted. } \\
\text { Accepted. }\end{array}$ & $\begin{array}{l}\text { Reworded as state } \\
\text { Accepted. Reword } \\
\text { Reworded as state }\end{array}$ & ated. & \\
\hline
\end{tabular}




\begin{tabular}{|l|l|l|l|}
\hline \multirow{2}{*}{ REVIEW COMMENT RECORD (RCR) } & $\begin{array}{l}\text { 1. Date } \\
2 / 12 / 98\end{array}$ & $\begin{array}{c}\text { Review No. } \\
200 E-002\end{array}$ \\
\cline { 2 - 4 } & $\begin{array}{l}\text { 3. Project No. } \\
\text { W-314 }\end{array}$ & $\begin{array}{l}4 . \quad \text { Page } \\
\text { of } 33\end{array}$ \\
\hline
\end{tabular}

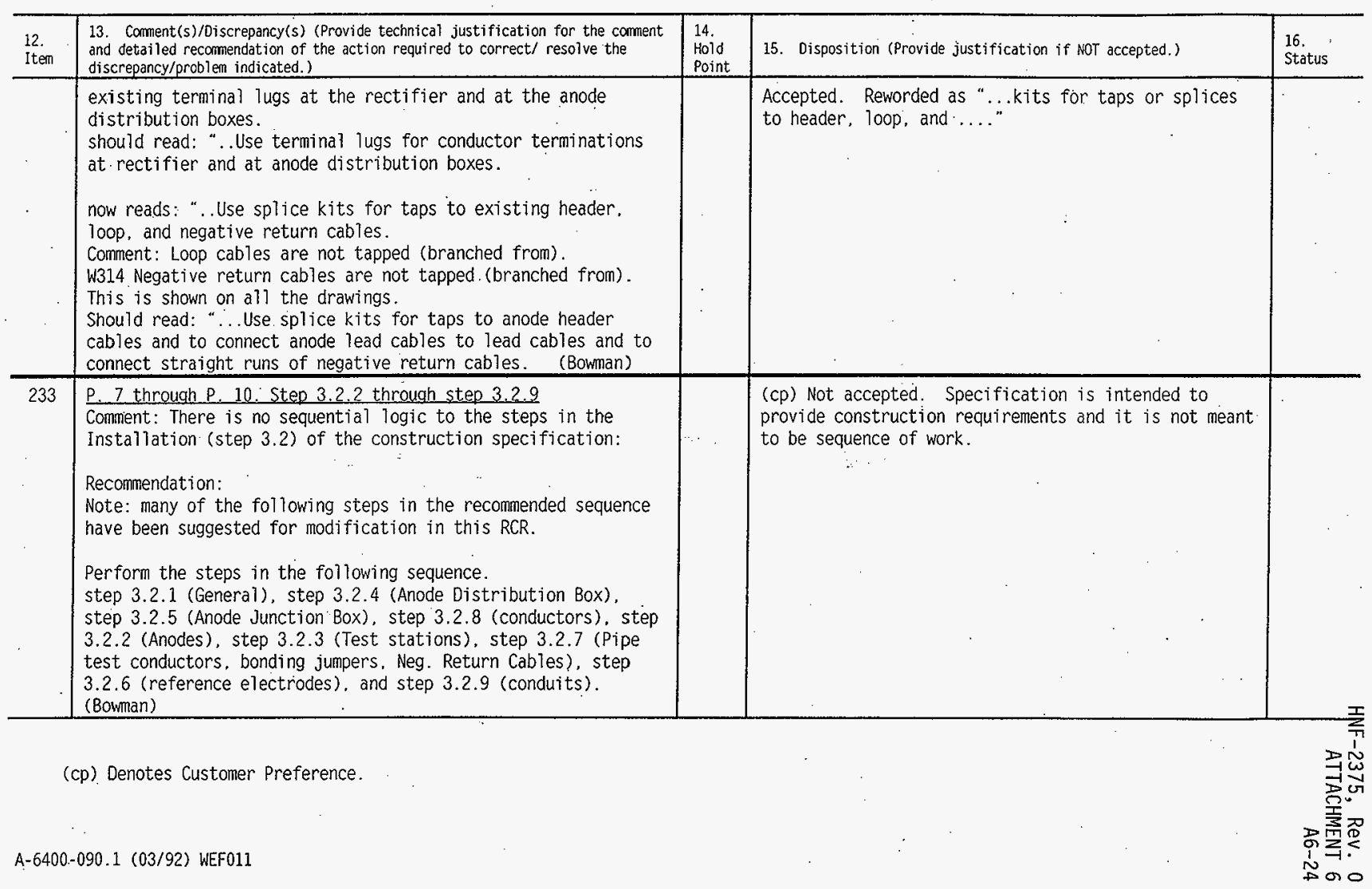




\section{anization)}

2. TO: CReceivin
DISTRIBUTION

Project $W-314 /$ TWRS

8. Originator Remarks:

This EDT releases for construction the approved design media (i.e., drawings and construction specification) associated with Project W-314's "200 East Upgrades" package. Design verification for these items is documented in the associated Design Review Report, HNF-2375.

11. Receiver Remarks: 11A. Design Baseline Document? [X] Yes [] No
3. From: (originating organization)
Tank Farm Restoration \& Safe Operations
6. Design Authority/ Design Agent/Cog. Engr.:
D. E. Bowers

5. Proj./Prog./Dept./Div.:

617948

\begin{tabular}{|c|}
\hline 4. Related EDr No.: \\
N/A
\end{tabular}

\begin{tabular}{|c|c|c|c|c|c|c|c|c|c|c|}
\hline 15. & \multicolumn{6}{|c|}{ DATA TRANSMITTED } & \multirow[b]{2}{*}{$\begin{array}{c}\text { (F) } \\
\text { Approval } \\
\text { Desig- } \\
\text { nator }\end{array}$} & \multirow[b]{2}{*}{$\begin{array}{c}\text { (G) } \\
\text { Reason } \\
\text { for } \\
\text { Trans- } \\
\text { mittal }\end{array}$} & \multirow[b]{2}{*}{$\begin{array}{c}\text { (H) } \\
\text { Origi- } \\
\text { nator } \\
\text { Dispo- } \\
\text { sition }\end{array}$} & \multirow[b]{2}{*}{$\begin{array}{l}\text { (1) } \\
\text { Receiv } \\
\text { or } \\
\text { Dispo- } \\
\text { sition }\end{array}$} \\
\hline $\begin{array}{l}\text { (A) } \\
\text { them } \\
\text { No. }\end{array}$ & \multicolumn{2}{|c|}{ (B) Document/Drawing No. } & $\begin{array}{c}\text { (C) } \\
\text { Sheet } \\
\text { No. }\end{array}$ & $\begin{array}{l}\text { (D) } \\
\text { Rev. } \\
\text { No. }\end{array}$ & \multicolumn{2}{|c|}{$\begin{array}{l}\text { (E) Title or Dascription of Data } \\
\text { Transmitted }\end{array}$} & & & & \\
\hline 1 & \multicolumn{2}{|l|}{$\mathrm{H}-14-100993$} & 1 & 0 & \multicolumn{2}{|c|}{$\begin{array}{l}\text { Drawing List - } \\
\text { Vicinity Map }\end{array}$} & ESQ & 2 & 1 & \\
\hline 2 & \multicolumn{2}{|l|}{$\mathrm{H}-14-100994$} & 1 & 0 & \multicolumn{2}{|c|}{$\begin{array}{l}\text { Civil Plan \& Profile } \\
-241-A N \text { to } 241-A Z\end{array}$} & ESQ & 2 & 1 & \\
\hline \multicolumn{11}{|l|}{16.} \\
\hline \multicolumn{2}{|c|}{ Approval Designator (F) } & \multicolumn{4}{|c|}{ Reason for Transmitral (G) } & \multicolumn{5}{|c|}{ Disposition (H) \& (I) } \\
\hline \multicolumn{2}{|c|}{$\begin{array}{l}E, S, 0, D \text { or } N / A \\
\text { (see WHC-CM-3-5, } \\
\text { Sec. 12.7) }\end{array}$} & $\begin{array}{l}\text { 1. Appr } \\
\text { 2. Roloe } \\
\text { 3. Inforr }\end{array}$ & \multicolumn{2}{|c|}{$\begin{array}{l}\text { 4. Reviow } \\
\text { 5. Post-Feview } \\
\text { 6. Dist. RReceipt }\end{array}$} & row. Required\} & \multicolumn{2}{|c|}{$\begin{array}{l}\text { 1. Approved } \\
\text { 2. Approved w/comment } \\
\text { 3. Disapproved w/comment }\end{array}$} & \multicolumn{3}{|c|}{$\begin{array}{l}\text { 4. Reviewed no/comment } \\
\text { 5. Reviewed w/comment } \\
\text { 6. Recoipt acknowledged }\end{array}$} \\
\hline
\end{tabular}

17. SIGNATUFE/DISTRIBUTION

(See Approval Designator for required signatures)

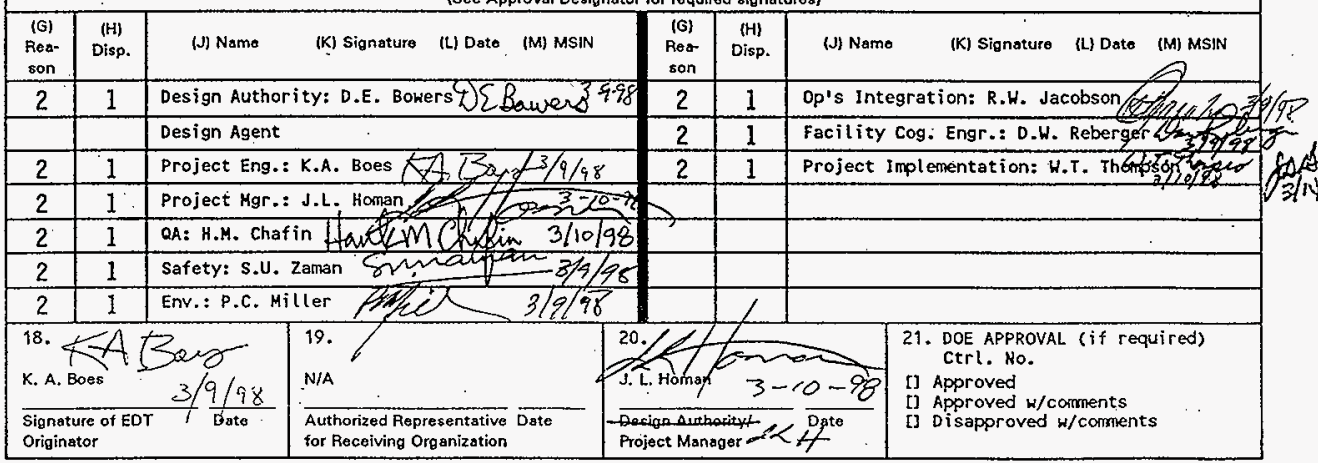

BD-7400-172-2 (05/96) GEFO97 
HNF-2375, Rev. 0, ATTACHMENT 7, A7-02

\begin{tabular}{|c|c|c|c|c|c|c|c|c|}
\hline \multicolumn{9}{|c|}{$\begin{array}{l}\text { ENGINEERING DATA TRANSMITTAL } \\
\text { (CONIINUATION PAGE) }\end{array}$} \\
\hline \multicolumn{2}{|c|}{$\begin{array}{l}\text { 5. Proj./Prog./Dept./Div.: } \\
\text { Project W-314 }\end{array}$} & & \multicolumn{2}{|c|}{$\begin{array}{l}\text { 6. Cog. Eng. } \\
\text { D. E. Bowers }\end{array}$} & $\begin{array}{l}\text { 1. } \\
\text { EOT } \\
617949 \\
\end{array}$ & & \multicolumn{2}{|c|}{ Page 2 of 8} \\
\hline 15. & & DAT & TRANSMIT & & (F) & (G) & (H) & (I) \\
\hline $\begin{array}{l}\text { (A) } \\
\text { Itom } \\
\text { No. }\end{array}$ & (B) Document/Drawing No. & $\begin{array}{l}\text { (C) } \\
\text { Sheot } \\
\text { No. }\end{array}$ & $\begin{array}{l}\text { (O) } \\
\text { Rov. } \\
\text { No. }\end{array}$ & $\begin{array}{l}\text { (E) Titio or Description of Data } \\
\text { Tranemitted }\end{array}$ & $\begin{array}{l}\text { Approvial } \\
\text { Dosig- } \\
\text { nator }\end{array}$ & $\begin{array}{c}\text { Reason } \\
\text { for } \\
\text { Trans- } \\
\text { mittal }\end{array}$ & $\begin{array}{l}\text { Origi- } \\
\text { nator } \\
\text { Dispo- } \\
\text { sition }\end{array}$ & $\begin{array}{l}\text { Receiv- } \\
\text { or } \\
\text { Dispo- } \\
\text { sition }\end{array}$ \\
\hline 3 & $\mathrm{H}-14-100995$ & 1 & 0 & $\begin{array}{l}\text { Civil Plan \& Profile } \\
241-A Z \text { to } 241-A X\end{array}$ & ESQ & 2 & 1 & \\
\hline 4 & $\mathrm{H}-14-100995$ & 2 & 0 & $\begin{array}{l}\text { Civil Plan \& Profile } \\
241-A Z \text { to } 241-A X\end{array}$ & ESQ & 2 & 1 & \\
\hline 5 & $H-14-100996$ & 1 & 0 & $\begin{array}{l}\text { Civil Plan \& Profile } \\
\text { 241-AX to } 241-A\end{array}$ & ESQ & 2 & 1 & \\
\hline 6 & $H-14-100996$ & 2 & 0 & $\begin{array}{l}\text { Civil Plan \& Profile } \\
241-A x \text { to } 241-A\end{array}$ & ESQ & 2 & 1 & \\
\hline 7 & $\mathrm{H}-14-100997$ & 1 & 0 & $\begin{array}{l}\text { Civil Plan \& Profile } \\
241-A X \text { to } 241-A Y\end{array}$ & ESQ & 2 & 1 & \\
\hline 8 & H-14-100998 & 1 & 0 & $\begin{array}{l}\text { Civil General Notes, } \\
\text { Legend and Typical } \\
\text { Sections }\end{array}$ & ESQ & 2 & 1 & $\overline{5}$ \\
\hline 9 & $\mathrm{H}-14-100999$ & 1 & 0 & $\begin{array}{l}\text { Structural Cover } \\
\text { Blocks 241-AX-B Plan }\end{array}$ & ESQ & 2 & 1 & \\
\hline 10 & $\mathrm{H}-14-101000$ & 1 & 0 & $\begin{array}{l}\text { Structural Cover } \\
\text { Blocks } 241-\mathrm{A}-\mathrm{B} \text { Plan }\end{array}$ & ESQ & 2 & 1 & \\
\hline 11 & $\mathrm{H}-14-101001$ & 1 & 0 & $\begin{array}{l}\text { Structural Cover } \\
\text { Blocks 24I-A \& AX } \\
\text { Sections and Details }\end{array}$ & ESQ & 2 & 1 & \\
\hline 12 & $\mathrm{H}-14-101002$ & 1 & 0 & $\begin{array}{l}\text { Structural } 241-A X-B \text { \& } \\
241-A-B \text { Wal1 } \\
\text { Modifications }\end{array}$ & ESQ & 2 & 1 & \\
\hline 13 & $H-14-101002$ & 2 & 0 & $\begin{array}{l}\text { Structural Pits 241- } \\
A X-B, \& \text { A-B Shield } \\
\text { Collar Plans }\end{array}$ & ESQ & 2 & 1 & \\
\hline 14 & $H-14-101002$ & 3 & 0 & $\begin{array}{l}\text { Structural Pits 241- } \\
A X-B, \& \text { A-B Shielding } \\
\text { Collar Details }\end{array}$ & ESQ & 2 & 1 & \\
\hline 15 & $\mathrm{H}-14-101002$ & 4 & 0 & $\begin{array}{l}\text { Structural Pits 241- } \\
\text { AX-B \& 24I-A-B Missie } \\
\text { Shield }\end{array}$ & ESQ & 2 & 1 & \\
\hline 16 & $\mathrm{H}-14-101008$ & 1 & 0 & $\begin{array}{l}\text { Electrical Site Plan } \\
\text { and Details } \\
\text { A/AN/AX/AY/AZ Tank } \\
\text { Farms }\end{array}$ & ESQ & 2 & 1 & \\
\hline 17 & $\begin{array}{c}\mathrm{H}-14-101008 \\
\vdots\end{array}$ & 2 & 0 & $\begin{array}{l}\text { Electrical Site Plan } \\
\text { and Details } \\
\text { A/AN/AX/AY/AZ Tank } \\
\text { Farms }\end{array}$ & ESQ & 2 & 1 & \\
\hline 18 & $\mathrm{H}-14-101009$ & 1 & 0 & $\begin{array}{l}\text { Electrical Power and } \\
\text { Control Plan Valve } \\
\text { Pit } 241-A X-B\end{array}$ & ESQ & 2 & 1 & \\
\hline
\end{tabular}


HNF-2375, Rev. 0, ATTACHMENT 7, A7-03

\begin{tabular}{|c|c|c|c|c|c|c|c|c|c|}
\hline \multicolumn{10}{|c|}{$\begin{array}{l}\text { ENGINEERING DATA TRANSMITTAL } \\
\text { (CONTINUATION PAGE) }\end{array}$} \\
\hline 5. $\mathrm{Pr}$ & $\begin{array}{l}\text { j./Pros./Dept./Div.: } \\
\text { Project H-314 }\end{array}$ & & $\begin{array}{r}\text { 6. Cog. En } \\
\text { D. }\end{array}$ & E. Bowers & 617949 & & Page 3 & of & \\
\hline 19 & $\mathrm{H}-14-101010$ & 1 & 0 & $\begin{array}{l}\text { Electrical } \\
\text { Installation Details } \\
\text { Valve Pit } 241-A X-B\end{array}$ & ESQ & 2 & 1 & & \\
\hline 20 & $\mathrm{H}-14-101010$ & 2 & 0 & $\begin{array}{l}\text { Electrical } \\
\text { Installation Details } \\
\text { Valve Pit 241-AX-B. }\end{array}$ & ESQ & 2 & 1 & & \\
\hline 21 & $\mathrm{H}-14-101011$ & 1 & 0 & $\begin{array}{l}\text { Electrical Panelboard } \\
\text { Schedule DP-AX-006 }\end{array}$ & ESQ & 2 & 1 & & \\
\hline 22 & H-14-101012 & 1 & 0 & $\begin{array}{l}\text { Electrical Wire Run } \\
\text { List Valve Pit 241- } \\
A X-B\end{array}$ & $E S Q$ & 2 & 1 & & \\
\hline 23 & $\mathrm{H}-14-101016$ & 1 & 0 & $\begin{array}{l}\text { Electrical Power and } \\
\text { Control Plan Valve } \\
\text { Pit } 241-A-B \\
\end{array}$ & ESQ & 2 & 1 & & \\
\hline 24 & $\mathrm{H}-14-101017$ & 1 & 0 & $\begin{array}{l}\text { Electrical } \\
\text { Installation Details } \\
\text { Valve Pit 241-A-B }\end{array}$ & ESQ & 2 & 1 & & \\
\hline 25 & $\mathrm{H}-14-101017$ & 2 & 0 & $\begin{array}{l}\text { Electrical. } \\
\text { Installation Details } \\
\text { Valve Pit } 241-A-B\end{array}$ & ESQ & 2 & 1 & & \\
\hline 26 & $\mathrm{H}-14-101018$ & 1 & 0 & $\begin{array}{l}\text { Electrical Panelboard } \\
\text { Schedule DP-A-015 }\end{array}$ & ESQ & 2 & 1 & & \\
\hline 27 & $\mathrm{H}-14-101019$ & 1 & 0 & $\begin{array}{l}\text { Electrical Hire Run } \\
\text { List Valve Pit } \\
241-A-B\end{array}$ & ESQ & 2 & 1 & & \\
\hline 28 & $\mathrm{H}-14-101020$ & 1 & 0 & $\begin{array}{l}\text { Cathodic Protection } \\
\text { Plan 241-AN-B to } 241- \\
\text { AZ-02A }\end{array}$ & ESQ & 2 & 1 & & \\
\hline 29 & $H-14-101020$ & 2 & 0 & $\begin{array}{l}\text { Cathodic Protection } \\
\text { Plan 241-AN-B to } 241- \\
\text { AZ-02A }\end{array}$ & ESQ & 2 & 1 & & \\
\hline 30 & $\mathrm{H}-14-101020$ & 3 & 0 & $\begin{array}{l}\text { Cathodic Protection } \\
\text { Plan } 241-A N-B \text { to } 241- \\
\text { AZ-O2A }\end{array}$ & ESQ & 2 & 1 & & \\
\hline 31 & $\begin{array}{c}H-14-101021 \\
\end{array}$ & 1 & 0 & $\begin{array}{l}\text { Cathodic Protection } \\
\text { Plan 241-AZ-02A to } \\
241-A X-B\end{array}$ & ESQ & .2 & 1 & & \\
\hline 32 & $\mathrm{H}-14-101021$ & 2 & 0 & $\begin{array}{l}\text { Cathodic Protection } \\
\text { Plan 24I-AZ-02A to } \\
241-A X-B\end{array}$ & ESQ & 2 & 1 & & \\
\hline 33 & $\mathrm{H}-14-101021$ & 3 & 0 & $\begin{array}{l}\text { Cathodic Protection } \\
\text { Plan 241-AZ-02A to } \\
241-A X-B\end{array}$ & ESQ & 2 & 1 & & \\
\hline 34 & $\mathrm{H}-14-101021$ & 4 & 0 & $\begin{array}{l}\text { Cathodic Protection } \\
\text { Plan } 241-A Z-02 A \text { to } \\
241-A X-B\end{array}$ & ESQ & 2 & 1 & & \\
\hline
\end{tabular}


HNF-2375, Rev. 0, ATTACHMENT 7, A7-04

\begin{tabular}{|c|c|c|c|c|c|c|c|c|c|}
\hline \multicolumn{10}{|c|}{$\begin{array}{l}\text { ENGINEERING DATA TRANSMITTAL } \\
\text { (CONTINUATION PAGE) }\end{array}$} \\
\hline \multicolumn{2}{|c|}{$\begin{array}{r}\text { 5. Proj./Prog./Dept./Div.: } \\
\text { Project W-314 }\end{array}$} & & \multicolumn{2}{|c|}{$\begin{array}{l}\text { 6. cog. Eng. } \\
\text { D. E. Bowers }\end{array}$} & $\begin{array}{r}\text { i. EDT } \\
617949 \\
\end{array}$ & \multicolumn{4}{|c|}{ Page 4 of $\frac{8}{7}$} \\
\hline 35 & $\mathrm{H}-14-101021$ & 5 & 0 & $\begin{array}{l}\text { Cathodic Protection } \\
\text { Plan } 241-A Z-02 A \text { to } \\
241-A X-B\end{array}$ & ESQ & 2 & 1 & & \\
\hline 36 & $\mathrm{H}-14-101021$ & 6 & 0 & $\begin{array}{l}\text { Cathodic Protection } \\
\text { PIan 241-AZ-02A to } \\
241-A X-B\end{array}$ & ESQ & 2 & 1 & & \\
\hline 37 & $\mathrm{H}-14-101022$ & 1 & 0 & $\begin{array}{l}\text { Cathodic Protection } \\
\text { Plan 241-AX-B to 241- } \\
A-B\end{array}$ & ESQ & 2 & 1 & & \\
\hline 38 & $\mathrm{H}-14-101022$ & 2 & 0 & $\begin{array}{l}\text { Cathodic Protection } \\
\text { Plan } 241-A X-B \text { to } 241- \\
A-B\end{array}$ & ESQ & 2 & 1 & & . \\
\hline 39 & $\mathrm{H}-14-101022$ & 3 & 0 & $\begin{array}{l}\text { Cathodic Protection } \\
\text { Plan 241-AX-B to } 241- \\
\text { A-B }\end{array}$ & ESQ & 2 & 1 & & \\
\hline 40 & H-14-101022 & 4 & 0 & $\begin{array}{l}\text { Cathodic Protection } \\
\text { Plan } 241-A X-B \text { to } 241- \\
A-B\end{array}$ & ESQ & 2 & 1 & & \\
\hline 41 & $\mathrm{H}-14-101022$ & 5 & 0 & $\begin{array}{l}\text { Cathodic Protection } \\
\text { Plan } 241-A X-B \text { to } 241- \\
A-B\end{array}$ & ESQ & 2 & 1 & & \\
\hline 42 & H-14-101023 & $I$ & 0 & $\begin{array}{l}\text { Cathodic Protection } \\
\text { Plan 241-AX-B to 241- } \\
\text { AY }-02 A\end{array}$ & $\mathrm{ESQ}$ & 2 & 1 & & \\
\hline 43 & $\mathrm{H}-14-101023$ & 2 & 0 & $\begin{array}{l}\text { Cathodic Protection } \\
\text { Plan } 241-A X-B \text { to } 241- \\
\text { AY }-02 A\end{array}$ & ESQ & 2 & 1 & & \\
\hline 44 & $\mathrm{H}-14-101023$ & 3 & 0 & $\begin{array}{l}\text { Cathodic Protection } \\
\text { Plan } 241-A X-B \text { to } 241- \\
\text { AY-02A }\end{array}$ & ESQ. & 2 & 1 & & \\
\hline 45 & $H-14-101024$ & 1 & 0 & $\begin{array}{l}\text { Cathodic Protection } \\
\text { Details }\end{array}$ & ESQ & 2 & 1 & & \\
\hline 46 & $\mathrm{H}-14-101024$ & 2 & 0 & $\begin{array}{l}\text { Cathodic Protection } \\
\text { Details }\end{array}$ & ESQ & 2 & 1 & & \\
\hline 47 & H-14-101028 & 1 & 0 & $\begin{array}{l}\text { INSTM Valve Pit } \\
\text { Upgrade Plan }\end{array}$ & ESQ & .2 & 1 & & \\
\hline 48 & H-14-101029 & 1 & 0 & $\begin{array}{l}\text { INSTM Valve Pit Plan } \\
241-A X-B\end{array}$ & ESQ & 2 & 1 & & \\
\hline 49 & $\mathrm{H}-14-101030$ & 1 & 0 & $\begin{array}{l}\text { INSTM } 241-A X-B \text { Valve } \\
\text { Pit Elevation }\end{array}$ & ESQ & 2 & 1 & & \\
\hline 50 & $\mathrm{H}-14-101031$ & 1 & 0 & $\begin{array}{l}\text { INSTM } 241-A X-B \text { Valve } \\
\text { Pit Leak Detection } \\
\text { Loop Diagram }\end{array}$ & $\mathrm{ESQ}$ & 2 & 1 & & \\
\hline 51 & H-14-101032 & 1 & 0 & $\begin{array}{l}\text { INSTM 24I-AX-B Enc } \\
\text { Low Pt. Leak Det Loop } \\
\text { Diagram }\end{array}$ & ESQ & 2 & 1 & & \\
\hline
\end{tabular}


HNF-2375, Rev. 0, ATTACHMENT 7, A7-05

ENGINEERING DATA TRANSMITTAL.

(CONTINUATION PAGE)

\begin{tabular}{|c|r|r|r|}
\hline $\begin{array}{c}\text { 5. Proj./Prog./Dept./Div.: } \\
\text { Project H-314 }\end{array}$ & $\begin{array}{r}\text { 6. Cog. Eng. } \\
\text { D. E. Bowers }\end{array}$ & $\begin{array}{r}\text { 1. EDT } \\
617949\end{array}$ & $\begin{array}{l}8 \\
\text { Page } 5 \text { of } 7\end{array}$ \\
\hline
\end{tabular}

\begin{tabular}{|c|c|c|c|c|c|c|c|c|}
\hline 52 & $H-14 \div 101033$ & 1 & 0 & $\begin{array}{l}\text { INSTM 241-AX-B Valve } \\
\text { Position Sensor Loop } \\
\text { Diagram }\end{array}$ & ESQ & -2 & 1 & \\
\hline 53 & $H-14-101033$ & 2 & 0 & $\begin{array}{l}\text { INSTM } 241-A X-B \text { Valve } \\
\text { Position Sensor Loop } \\
\text { Diagram }\end{array}$ & ESQ & 2 & 1 & \\
\hline 54 & $\mathrm{H}-14-101034$ & 1 & 0 & $\begin{array}{l}\text { INSTM 241-AX-B } \\
\text { Instrument List }\end{array}$ & ESQ & 2 & 1 & \\
\hline 55 & $H-14-101034$ & 2 & 0 & $\begin{array}{l}\text { INSTM } 241-A X-B \\
\text { Instrument List }\end{array}$ & ESQ & 2 & 1 & \\
\hline 56 & $\mathrm{H}-14-101035$ & 1 & 0 & $\begin{array}{l}\text { INSTM Valve Pit Plan } \\
241-A-B \text {. }\end{array}$ & ESQ & 2 & 1 & \\
\hline 57 & $\mathrm{H}-14-101036$ & $I$ & 0 & $\begin{array}{l}\text { INSTM 241-A-B Vajve } \\
\text { Pit Elevation }\end{array}$ & ESQ & 2 & 1 & \\
\hline 58 & H-14-101037 & 1 & 0 & $\begin{array}{l}\text { INSTM 241-A-B Valve } \\
\text { Pit Leak Detection } \\
\text { Loop Diagram }\end{array}$ & ESQ & 2 & 1 & \\
\hline 59 & $H-14-101038$ & 1 & 0 & $\begin{array}{l}\text { INSTM Valve Position } \\
\text { Sensor Loop Diagram }\end{array}$ & ESQ & 2 & 1 & \\
\hline 60 & $H-14-101038$ & 2 & 0 & $\begin{array}{l}\text { INSTM Valve Position } \\
\text { Sensor Loop Diagram }\end{array}$ & ESQ & 2. & 1 & \\
\hline 61 & $\mathrm{H}-14-101039$ & 1 & 0 & INSTM Instrument List & ESQ & 2 & 1 & \\
\hline 62 & $H-14-101039$ & 2 & 0 & INSTM Instrument List & ESQ & 2 & 1 & \\
\hline 63 & $H-14-101040$ & 1 & 0 & $\begin{array}{l}\text { INSTM A-Farm Field } \\
\text { Terminal Box } \\
\text { Interconnection } \\
\text { Diagram } \\
\end{array}$ & ESQ & 2 & 1 & \\
\hline 64 & $\mathrm{H}-14-101041$ & 1 & 0 & $\begin{array}{l}\text { INSTM AX-Farm FieId } \\
\text { Terminal Box } \\
\text { Interconnection } \\
\text { Diagram }\end{array}$ & ESQ & 2 & 1 & \\
\hline 65 & $\mathrm{H}-14-101047$ & 1 & 0 & $\begin{array}{l}\text { P\&ID Va]ve Pit 241- } \\
\text { AX-B }\end{array}$ & ESQ & 2 & 1 & \\
\hline 66 & $\mathrm{H}-14-101048$ & 1 & 0 & $\begin{array}{l}\text { P\&ID Miscellaneous } \\
\text { Details }\end{array}$ & ESQ & 2 & 1 & \\
\hline 67 & $\mathrm{H}-14-101051$ & 1 & 0 & $\begin{array}{l}\text { Jumper Arrangement } \\
\text { Vaive Pit } 241-A X-B\end{array}$ & ESQ & 2 & 1 & \\
\hline 68 & $\mathrm{H}-14-101052$ & 1 & 0 & $\begin{array}{l}\text { Jumper Arrangement } \\
\text { Valve Pit } 241-A X-B \\
\text { Section }\end{array}$ & ESQ & 2 & 1 & \\
\hline 69 & H-14-101061 & 1 & 0 & $\begin{array}{l}\text { Jumper Assembly 241- } \\
A X-B \text { RI-R20-(D\&E) }\end{array}$ & ESQ & 2 & 1 & \\
\hline 70 & $\mathrm{H}-14-101061$ & 2 & 0 & $\begin{array}{l}\text { Jumper Assembly } 241- \\
A X-B \text { R1-R20-(D\&E) }\end{array}$ & ESQ & 2 & 1 & \\
\hline 71 & $\mathrm{H}-14-101062$ & 1 & 0 & $\begin{array}{l}\text { Jumper Assembly 241- } \\
A X-B \text { R12-(D) }\end{array}$ & $\mathrm{ESQ}$ & 2 & 1 & \\
\hline 72 & H-14-101064 & 1 & 0 & $\begin{array}{l}\text { Jumper Assembly 241- } \\
\text { AX-B RlA-Drain }\end{array}$ & ESQ & 2 & 1 & \\
\hline
\end{tabular}


HNF-2375, Rev. 0, ATTACHMENT 7, A7-06

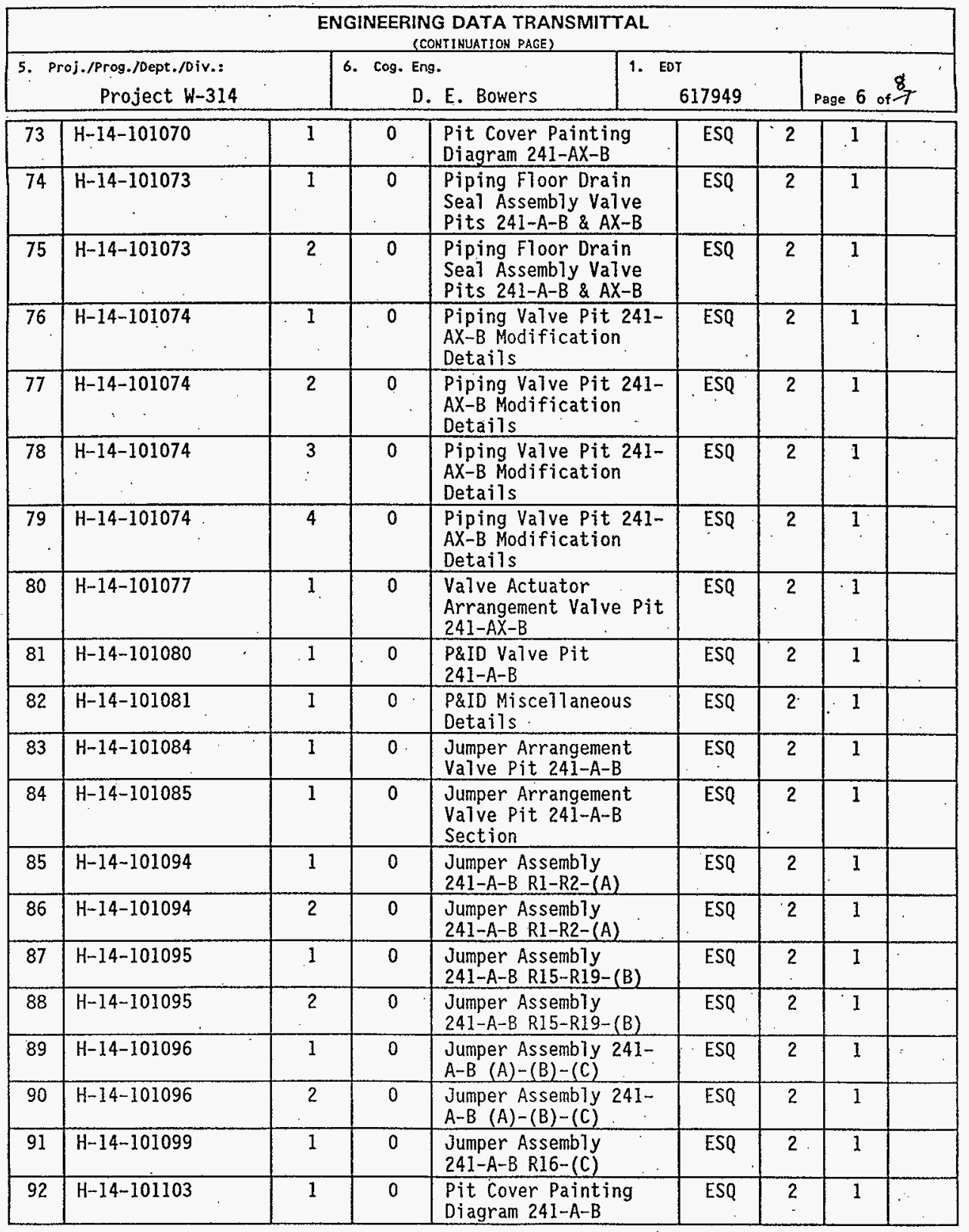


HNF-2375, Rev. 0, ATTACHMENT 7, A7-07

ENGINEERING DATA TRANSMITTAL.

(CONTINUATIOH PAGE)

5. Proj./Prog./Dept./Div.: 6. Cog. Eng.

D. E. Bowers

1. EDT

Project $\mathrm{H}-314$

Page 7 of 8

\begin{tabular}{|c|c|c|c|c|c|c|c|c|}
\hline 93 & $\mathrm{H}-14-101104$ & 1 & 0 & $\begin{array}{l}\text { Valve Actuator } \\
\text { Arrangment Valve Pit } \\
241-A-B\end{array}$ & $\mathrm{ESQ}$ & 2 & 1 & \\
\hline 94 & $H-14-101105$ & 1 & 0 & $\begin{array}{l}\text { Piping Valve Pit } 241- \\
\text { A-B Modification } \\
\text { Details }\end{array}$ & ESQ & 2 & 1 & \\
\hline 95 & $\mathrm{H}-14-101110$ & 1 & 0 & $\begin{array}{l}\text { Piping Plan } 241-A N-B \\
\text { to } 241-A Z-02 A\end{array}$ & ESQ & 2 & 1 & \\
\hline 96 & $\mathrm{H}-14-101110$ & 2 & 0 & $\begin{array}{l}\text { Piping Plan 241-AN-B } \\
\text { to } 241-A Z-02 A\end{array}$ & ESQ & 2 & 1 & . \\
\hline 97 & $\mathrm{H}-14-101110$ & 3 & 0 & $\begin{array}{l}\text { Piping PTan } 241-A N-B \\
\text { to } 241-A Z-02 A\end{array}$ & ESQ & 2 & 1 & \\
\hline 98 & $\mathrm{H}-14-101111$ & 1 & 0 & $\begin{array}{l}\text { Piping Support Plan } \\
3^{\prime \prime} S N-630-M 9\end{array}$ & ESQ & 2 & 1 & \\
\hline 99 & $\mathrm{H}-14-101112$ & 1 & 0 & Piping Details & ESQ & 2 & 1 & \\
\hline 100 & $\mathrm{H}-14-101113$ & 1 & 0 & $\begin{array}{l}\text { Piping Plan } 241- \\
A Z-02 A \text { to } 241-A X-B \text {. }\end{array}$ & ESQ & 2 & 1 & \\
\hline 101 & $H-14-101113$ & 2 & 0 & $\begin{array}{l}\text { Piping Plan 24l- } \\
A Z-02 A \text { to } 241-A X-B\end{array}$ & ESQ. & 2 & 1 & \\
\hline 102 & $H-14-101113$ & 3 & 0 & $\begin{array}{l}\text { Piping Plan 241- } \\
A Z-02 A \text { to } 241-A X-B\end{array}$ & ESQ & 2 & 1 & \\
\hline 103 & $\mathrm{H}-14-101113$ & 4 & 0 & $\begin{array}{l}\text { Piping Plan 241- } \\
\text { AZ-02A to } 241-A X-B\end{array}$ & ESQ & 2 & 1 & \\
\hline 104 & $\mathrm{H}-14-101113$ & 5 & 0 & $\begin{array}{l}\text { Piping Plan } 241- \\
A Z-02 A \text { to } 241-A X-B\end{array}$ & ESQ & 2 & 1 & \\
\hline 105 & $\mathrm{H}-14-101113$ & 6 & 0 & $\begin{array}{l}\text { Piping Plan } 241- \\
A Z-02 A \text { to } 241-A X-B\end{array}$ & $\overline{E S Q}$ & 2 & 1 & \\
\hline 106 & $\mathrm{H}-14-101114$ & 1 & 0 & $\begin{array}{l}\text { Piping Support Plan } \\
3^{\prime \prime} \text { SN-632-M9 }\end{array}$ & ESQ & 2 & 1 & \\
\hline 107 & $\mathrm{H}-14-101116$ & 1 & 0 & $\begin{array}{l}\text { Piping Plan } 241-A X-B \\
\text { to } 241-A-B\end{array}$ & ESQ & 2 & 1 & \\
\hline 108 & $\mathrm{H}-14-101116$ & 2 & 0 & $\begin{array}{l}\text { Piping Plan 241-AX-B } \\
\text { to } 241-A-B\end{array}$ & ESQ & 2 & 1 & \\
\hline 109 & $\mathrm{H}-14-101116$ & 3 & 0 & $\begin{array}{l}\text { Piping Plan 241-AX-B } \\
\text { to } 24 \mathrm{I}-\mathrm{A}-\mathrm{B}\end{array}$ & ESQ & 2 & 1 & \\
\hline 110 & $H-14-101116$ & 4 & 0 & $\begin{array}{l}\text { Piping Plan } 241-A X-B \\
\text { to } 241-A-B\end{array}$ & ESQ & 2 & I & \\
\hline 111 & $\mathrm{H}-14-101116$ & 5 & 0 & $\begin{array}{l}\text { Piping Plan } 241-A X-B \\
\text { to } 241-A-B\end{array}$ & $\overline{E S Q}$ & 2 & 1 & \\
\hline 112 & $H-14-101117$ & 1 & 0 & $\begin{array}{l}\text { Piping Support Plan } \\
3^{\prime \prime} 5 N-634-M 9\end{array}$ & ESQ & 2 & 1 & \\
\hline 113 & $H-14-101117$ & 2 & 0 & $\begin{array}{l}\text { Piping Support Plan } \\
3^{\prime \prime} \text { SN-634-M9 }\end{array}$ & $\mathrm{ESQ}$ & 2 & 1 & \\
\hline 114 & $\mathrm{H}-14-101117$ & 3 & 0 & $\begin{array}{l}\text { Piping Support P1an } \\
3^{\prime \prime} \text { SN-634-M9 }\end{array}$ & ESQ & 2 & 1 & \\
\hline 115 & $H-14-101118$ & 1 & 0 & $\begin{array}{l}\text { Piping Plan 241-AX-B } \\
\text { to } 241-A Y-02 A\end{array}$ & ESQ & 2 & 1 & \\
\hline
\end{tabular}


HNF-2375, Rev. 0, ATTACHMENT 7, A7-08

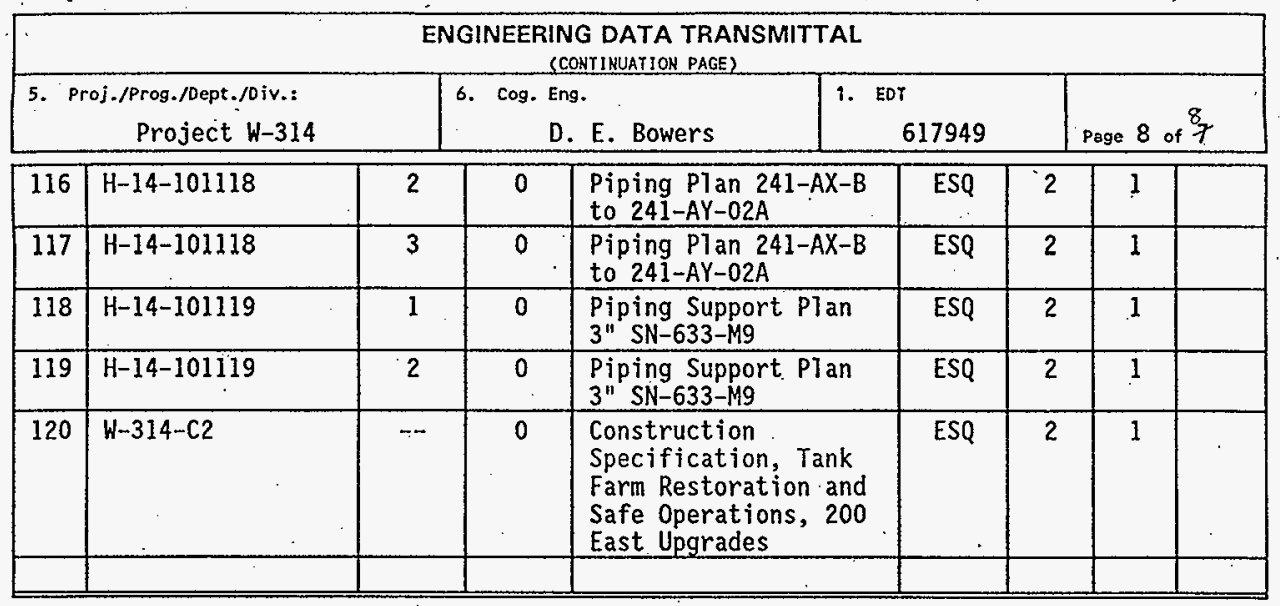




\section{DISTRIBUTION SHEET}

\begin{tabular}{|c|c|c|c|c|c|}
\hline \multirow{2}{*}{$\begin{array}{l}\text { To } \\
\text { Distribution }\end{array}$} & \multirow{2}{*}{\multicolumn{3}{|c|}{$\begin{array}{l}\text { From } \\
\text { K. A. Boes }\end{array}$}} & \multicolumn{2}{|l|}{ Page 1 of 1} \\
\hline & & & & \multicolumn{2}{|c|}{ Date Apri1 10, 1998} \\
\hline \multicolumn{4}{|l|}{ Project Title/Work Order } & \multicolumn{2}{|c|}{ EDT No. 624066} \\
\hline \multicolumn{4}{|c|}{$\begin{array}{l}\text { W-314, Tank Farm Restoration and Safe Operations, } 200 \text { East } \\
\text { Upgrades, Design Review Report, HNF-2375 }\end{array}$} & \multicolumn{2}{|l|}{ ECN No. } \\
\hline Name & MSIN & $\begin{array}{c}\text { Text } \\
\text { With Al! } \\
\text { Attach. }\end{array}$ & Text Only & $\begin{array}{l}\text { Attach./ } \\
\text { Appendix } \\
\text { Only }\end{array}$ & $\begin{array}{l}\text { EDT/ECN } \\
\text { Only }\end{array}$ \\
\hline
\end{tabular}

M. G. A7-Wazani

K. A. Boes

T. W. Bohan

D. E. Bowers

H. M. Chafin

D. B. Cole

R. A. Dodd

J. D. Galbraith

J. L. Gilbert

P. A. Haine

C. E. Hatch

W. H. Hays

G. A. Hester

J. L. Homan

R. W. Jacobson

J. R. LaPointe

R. L. Legg

J. T. Koberg

D. L. McGrew

P. C. Miller

R. S. Nichoison

D. R. Nunamaker

J. E. Pieper

T. K. Ravencraft

D. W. Reberger

M. D. Rickenbach

S. H. Rifaey

C. C. Scajef

M. A. Scott

G. Singh

W. T. Thompson

M. W. Tiffany

T. L. Warnick

S. U. Zaman

Project Files

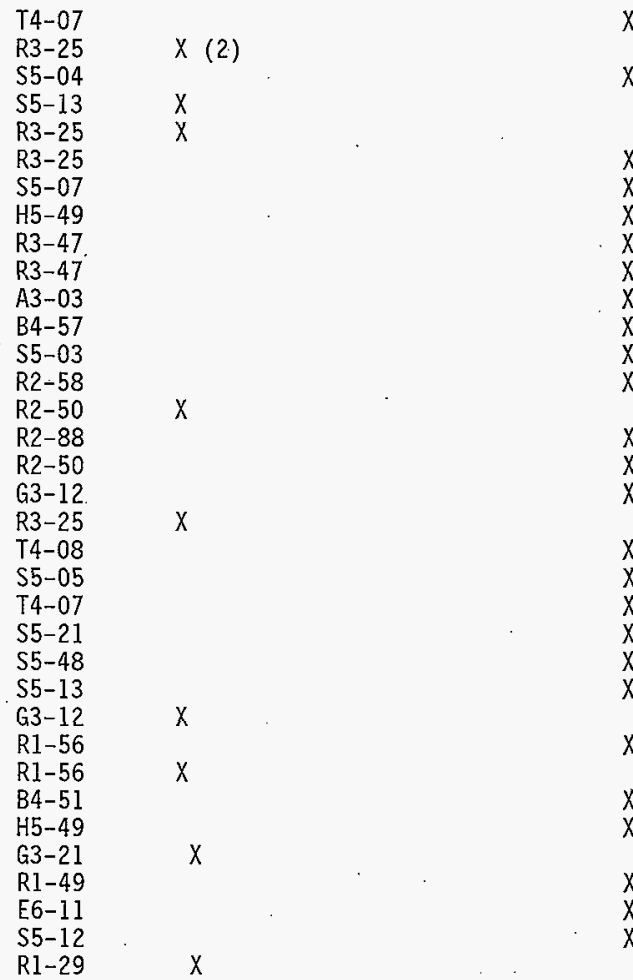

$x$

$x$

$x$

$x$

$x$

$x$

$x$

$x$

$x$

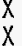

$x$

$x^{-}$

$x$

$x$

$x$

$x$

$x$

$X$

$x$

$x$

$x$

$x$
$x$ 